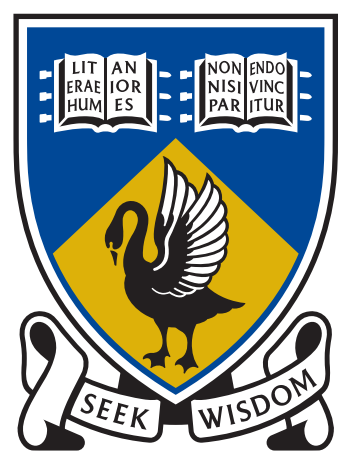

\title{
THE UNIVERSITY OF WESTERN AUSTRALIA
}

Achieve International Excellence

\section{POWER CONSUMPTION OPTIMIZATION IN \\ DIGITAL IMAGE PROCESSING MODULES FOR \\ IMAGE SENSOR APPLICATIONS

\author{
by \\ Xiaoxiao ZHANG \\ Master of philosophy, IME of Chinese Academy of Science
}

This thesis is presented for the degree of

Doctor of Philosophy of

The University of Western Australia

School of Electrical, Electronic and Computer Engineering

The University of Western Australia 


\section{Acknowledgements}

First of all, I wish to express my hearty gratitude to my supervisor, Prof. Farid Boussaid, who has given me tremendous support throughout my postgraduate study. His profound understanding in CMOS sensor fields and systematic research methodologies have benefited me a lot in identifying and analyzing potential research problems and opportunities. Apart from sharing his invaluable knowledge and experience, his constant motivation and encouragement have always been of great importance towards my research work. His effort in maintaining an open research environment has facilitated me to broaden my thinking both academically and personally.

I am deeply indebted to my supervisor in HKUST, Prof. Amine BERMAK, for all of his technical, financial, and personal support during my years at HKUST. He has been patiently guiding me and enthusiastically encouraging me throughout these four years. He developed my skills of identifying research problems and finding solutions precisely and clearly. What I have learned from him will benefit me well beyond my graduation in my future research career and personal life.

I would like to thank all of the wonderful friends in UWA Microelectronics Research Group. They have helped me in many ways during my study at UWA.

Most of all, I am grateful to my whole family for their devotion, understanding, and support. They are the most important source of my inspiration, encouragement, and happiness. 


\section{Abstract}

Recent advances in semiconductor fabrication technology have enabled the concept of a single camera-on-a-chip, which integrates all camera functions onto a single piece of silicon. To enable the concept of low cost and high performance miniature camera-on-a-chip, the proposed research aims at developing advanced real-time digital image processing (DIP) cores or modules that could be integrated together with the photo-sensing pixel array. The developed DIP cores address the stringent requirements of compactness, low-power, and real-time operation to enable their integration into a variety of low cost portable consumer imaging products.

This work first presents a multi-precision reconfigurable multiplier, which incorporates variable precision, parallel processing, razor-based dynamic voltage scaling (DVS), and dedicated multi-precision operands scheduling to realize full energy and performance flexibility and efficiency. According to user's arbitrary requirements (eg. throughput), the dynamic voltage and frequency scaling management unit first configures the multiplier operating at the proper precision and frequency. Adapting to the run-time workload of the targeted application, razor flip-flops and the dithering voltage unit assist the voltage and frequency scaling management units to autonomously configure the multiplier to work at the minimum possible power operating point to achieve the minimized power consumption. This multi-precision multiplier is coupled with an operands scheduler, which can analyze and rearrange the input data to achieve the optimal voltage and frequency combinations to further reduce the overall power consumption. Our work successfully demonstrates through a fabricated prototype that multi-precision architecture can reap the benefits from dynamic voltage scaling techniques more effectively, which enables the efficient use for DIP applications.

A new digital image sensor (DPS) architecture based on a multi-reset integration scheme was proposed to reduce memory needs, sensor size and consumption of the pixel level. The operation of the DPS exploits the chronological change of the code. In the proposed implementation, a 4-bit in-pixel memory is used 
to reduce the pixel size, and an 8-bit resolution is achieved with the multi-reset scheme. In addition, full complementary metal-oxide-semiconductor (CMOS) 2T DRAM and selective refresh scheme are adopted to implement the memory elements and maximize the area savings. The proposed architecture was validated by a prototype chip fabricated using AMS 0.35 $\mu \mathrm{m}$ CMOS technology. Our scheme achieves a $20 \%$ fill factor for a $22 \mu m \times 22 \mu m$ digital pixel sensor, with a power consumption per pixel reduced by around 37.5\% compared to previous implementations.

Finally, we reduced image processing precision to 1 single bit. The resulting binary images and corresponding Boolean operations reduce the computational complexity of DIP cores/modules significantly compared to traditional fullprecision arithmetic processing. The proposed approach exploits the typically long integration times of each image plane in order to carry out processing at low operating frequency and supply voltage. To validate our proposed scheme, a novel bit-plane-based local-voting early-stop Hough transform algorithm was implemented on a Xilinx Virtex-4 FPGA board. Experimental results demonstrate that our proposed algorithm greatly reduces the computation load, improves the processing efficiency and reduces the power consumption while maintaining the accuracy to an acceptable level. Our parallel architecture results in an 8 times speedup and $16 \%$ memory reduction compared to traditional architectures. It also provides up to 31 times processing efficiency improvement compared with prior works. 


\section{TABLE OF CONTENTS}

Acknowledgements

Abstract

1 Introduction $\quad \mathbf{1}$

1.1 Background and Motivation ... . . . . . . . . . 1

1.2 Statement of Research Objectives and Contribution . . . . . . . . 3

1.3 Thesis Organization . . . . . . . . . . . . . . . . . . . . . 4

2 Optimization of Multiplier for Low-Power DIP Cores 5

2.1 Introduction . . . . . . . . . . . . . . . . . 5

2.2 Wordlength and Voltage Optimization 6

2.3 Design of Multi-precision DVS Low-Power Multiplier . . . . . . 8

2.3.1 System Overview . . . . . . . . . . . . . . . . 8

2.3.2 Multi-precision and Reconfigurability Overhead . . . . . 12

2.3.3 Dynamic Voltage and Frequency Scaling Management . . . 16

2.3.4 Implementation of Razor Flip-flops . . . . . . . . . . . . . 19

2.4 Performance Evaluation and Discussion . . . . . . . . . . . . . . . 21

2.5 Summary . . . . . . . . . . . . . . . . . . . 26

3 Dedicated Operands Scheduler for Optimal Operation Voltages $\begin{array}{ll}\text { and Frequencies } & 27\end{array}$

3.1 Introduction . . . . . . . . . . . . . . . . . . . 27

3.2 Algorithms and Performance Comparison . . . . . . . . . . . . 31

3.2.1 Algorithm A for Low Cost Implementation . . . . . . . . . 31

3.2.2 Algorithm B for Zero Voltage Transition . . . . . . . . . . 32

3.2.3 Algorithm C for Minimum Power . . . . . . . . . . . . . . 33

3.2.4 Scheduling Overhead Evaluation . . . . . . . . . . . 34

3.2.5 Potential Applications . . . . . . . . . . . . . 35

3.3 Summary . . . . . . . . . . . . . . . . 35 
4 A Compact Multi-Reset Integration Digital Pixel Sensor

4.1 Introduction . . . . . . . . . . . . . . . . . . . 37

4.2 Time-Domain PWM DPS . . . . . . . . . . . . . . . . 39

4.2.1 Conventional Architecture . . . . . . . . . . . . . . 39

4.2 .2 Conversion Time Analysis . . . . . . . . . . . . . . . . . 42

4.3 The Proposed Multi-Reset Integration

(MRI) Scheme . . . . . . . . . . . . . . . . . . . 45

4.3 .1 MRI Concept . . . . . . . . . . . . . . . 46

4.3.2 Trade-off Analysis . . . . . . . . . . . . . . . . . . . . . . . 48

4.3 .3 Overall Architecture . . . . . . . . . . . . . . . . . . . . 49

4.3 .4 Pixel Circuit . . . . . . . . . . . . . . . 51

4.3.5 2T DRAM Implementation . . . . . . . . . . . 53

4.3.6 Differential sensing scheme and voltage generation . . . . 54

4.3.7 Read and Refresh Circuits . . . . . . . . . . . . . 55

4.4 Power Analysis and Power Reduction Techniques . . . . . . . . 58

4.4.1 Power Consumption Analysis . . . . . . . . . . . . . . 58

4.4.2 Power Reduction Techniques . . . . . . . . . . . . . 59

4.5 Hardware and Measurement Results . . . . . . . . . . . . . . . 60

4.6 Summary . . . . . . . . . . . . . . . . . . 64

5 Bit-Plane Based Hough Transform Processing for Real-Time Image Segmentation $\quad 65$

5.1 Introduction . . . . . . . . . . . . . . . . . . 65

5.2 Hough Transform . . . . . . . . . . . . . . . . . . . . . 68

5.3 Proposed Bit-Plane Based Early-Stop Hough Transform . . . . . . 74

5.3.1 Overall Algorithm Description . . . . . . . . . . . 75

5.3.2 Hardware Optimization and Implementation . . . . . . . . 84

5.3.3 Experimental Results and Analysis . . . . . . . . . . . 90

5.4 Summary . . . . . . . . . . . . . . . . . . . . . 92

6 Conclusion and Future Work 93

$\begin{array}{ll}\text { Bibliography } & 96\end{array}$ 


\section{List of Figures}

2.1 Multiplier system architecture. . . . . . . . . . . . . . 9 9

2.2 Normalized delay, minimal voltage, and power consumption as a function of the bit-width of the multiplier. . . . . . . . . . . 10

2.3 Possible configuration modes of the proposed multi-precision multiplier. . . . . . . . . . . . . . . . . 11

2.4 Optimization space of multi-precision, DVS, and parallel processing approaches. . . . . . . . . . . . . . . . 12

2.5 Structure of the input interface unit. . . . . . . . . . . . 13

2.6 How three processing elements (PEs) can be combined to form a 16bit $\times 16$ bit multiplier. . . . . . . . . . . . . . . . . . 14

2.7 DVS arithmetic logic using two header switches for voltage dithering. 17

2.8 Transient Vdd switching current. . . . . . . . . . . . . . . 17

2.9 (a) The proposed single-header voltage dithering unit and the voltage and frequency tuning loops. (b) Experimental results of timing of the voltage dithering unit. . . . . . . . . . . . . . 18

2.10 Output clock frequency as a function of control bits sequence for different process corners. . . . . . . . . . . . . . . . . . 19

2.11 Experimental measurement of the worst case frequency switching (from $50 \mathrm{MHz}$ to $5 \mathrm{MHz}$ ). . . . . . . . . . . . . 20

2.12 Conceptive view of the Razor flip-flop. . . . . . . . . . . . 21

2.13 Microphotograph of the $32 \times 32$-bit multi-precision multiplier. . . . 22

2.14 Experimental measurements of the minimum voltage supply for different precisions and operating frequencies. . . . . . . . .

2.15 Experimental results of power consumption of different operating schemes. (MP: Multi-precision; DVS: Dynamic voltage scaling; PP: Parallel-processing) . . . . . . . . . . . . . .

2.16 Experimental data of power savings associated to multi-precision, razor-based DVS and parallel processing schemes. (MP: Multiprecision; DVS: Dynamic voltage scaling; PP: Parallel-processing)

3.1 Block diagram of the input operands scheduler. . . . . . . . . . . 28 
3.2 Principles of algorithm A, B and C. . . . . . . . . . . . 32

4.1 Schematic view of the conventional pixel. . . . . . . . . . . . . 40

4.2 The timing diagrams of the conventional pixel. . . . . . . . . . . . 40

4.3 Uniform time domain quantization. . . . . . . . . . . . . . . . . . 41

4.4 Non-uniform time domain quantization. . . . . . . . . . . . . . 42

4.5 3bits UQ and NUQ in terms of time. . . . . . . . . . . . . 44

4.6 3bits UQ and NUQ in terms of discharging current. . . . . . . . . 44

4.7 Timing diagram of the multi-reset integration scheme. . . . . . . . 46

4.8 Illustration of timing boundaries for a 2-bit resolution. . . . . . . 48

4.9 Pixel area as function of the number of embedded bits. . . . . . . 49

4.10 Interpolated curve of the delay as a function of memory size. . . . 50

4.11 Block diagram of the overall architecture. . . . . . . . . . . . . . 51

4.12 Pixel schematic and layout. . . . . . . . . . . . . . . . 52

4.13 Simulated timing diagram illustrating the pixel's operation. . . . . 52

4.14 2T-DRAM cell. . . . . . . . . . . . . . . . 53

4.15 2T-DRAM cell Operating principle. . . . . . . . . . . . 54

4.16 Diagram of the transposed bit-line architecture and the latching stage. . . . . . . . . . . . . . . . 55

4.17 Schematic view of the replica scheme. . . . . . . . . . . . 56

4.18 Diagram of the reference voltage generation. . . . . . . . . . 57

4.19 Schematic diagram of the memory read and refresh scheme. . . . . 57

4.20 Simulated Curves. . . . . . . . . . . . . . . . . . . . . . 58

4.21 Schematic diagram of the proposed switched-opamp comparator for PWM DPS applications. . . . . . . . . . . . . . . . . 61

4.22 Simulation results at nominal process and supply. . . . . . . . . 61

4.23 Microphotograph of the prototype. . . . . . . . . . . . . . 62

4.24 Measurement results of key electrical signals. Reset, Vpd, Wbl and $\mathrm{Rbl}$ are the reset signal, the photodiode voltage, the right bit line signal and the read bit line signal, respectively. . . . . . . . .

5.1 The four most significant binary (left column) and four least significant binary (right column) bit planes of an image. . . . . . . 66

5.2 The concept of Hough transform for line detection. . . . . . . . . 70

5.3 The Hough transform of a straight line. (a)Image space. (b)Parameter space. . . . . . . . . . . . . . . 71 
5.4 Gradient of the image. . . . . . . . . . . . . . . . 72

5.5 Parameter space. . . . . . . . . . . . . . . . . . 74

5.6 The timing diagram of the proposed multi-reset integration sensor. 76

5.7 The utilization rate of the accumulating array. . . . . . . . 78

5.8 The lines found in the parameter space. . . . . . . . . . . . . . 78

5.9 Stages of the proposed Hough algorithm. . . . . . . . . . . . . . . 80

5.10 Performance comparison of different Hough transform schemes. Column(a): original images; column(b): traditional full-precision global-voting algorithm; column(c): our proposed bit-plane earlystop global-voting algorithm; column(d): our proposed bit-plane early-stop local-voting algorithm. . . . . . . . . . . . . . 83

5.11 Block diagram of the Hough transform hardware implementation. 85

5.12 The row buffer scheme for the parallel gradient computation. (a)The data sequence of the gradient computation. (b)The proposed buffer switching scheme for power reduction. . . . . . . . . . . 86

5.13 The parallel hardware architecture of our Hough transform algorithm. . . . . . . . . . . . . . . 89

5.14 Experimental results of proposed algorithm. (a)Traffic Signs Detection. (b)Handwritten Alphanumeric Characters Recognition. 


\section{List of Tables}

2.1 Area and Power Comparison of the proposed multi-precision multipliers and traditional fixed-width Multipliers running at $50 \mathrm{MHz}$

2.2 Prototype Characteristics . . . . . . . . . . . . . . . . . 22

4.1 Power consumption analysis from electrical simulation at nominal process and supply voltage. . . . . . . . . . . . . . . 59

4.2 Comparison of key metrics with related work. . . . . . . . . 63

5.1 The clock cycle entry for different counter range. . . . . . . . . . 76

5.2 The summary of the logic utilization of the FPGA device. . . . . 91

5.3 Performance Comparison with Related Works . . . . . . . . . . . 91 


\section{List of Abbreviations}

CMOS Complementary Metal-Oxide-Semiconductor

DSP Digital Signal Processor

DVFS Dynamic Voltage and Frequency Scaling

DVS Dynamic Voltage Scaling

DFS Dynamic Frequency Scaling

VCO Voltage controlled Oscillator

PE Processing Element

SIMD Single Instruction Multiple Data

MSB Most Significant Bit

LSB Least Significant Bit

MP Multi-Precision

PP Parallel Processing

FPGA Field Programmable Gate Array

VLSI Very Large Scale Integration

IPC Instruction Per Cycle

DIP Digital Image Processing

OS Operands Scheduler

DPS Digital Pixel Sensor

SRAM Static Random-access Memory

DRAM Dynamic Random-access Memory

CCD Charge-coupled Device

PFM Pulse Frequency Modulation

PWM Pulse Width Modulation

AER Address Event Representation

SNR Signal-to-noise Ratio

UQ Uniform Quantization

NUQ Non-uniform Quantization

MRI Multi-reset Integration (MRI)

RGB Red, Green, and Blue

DA Distributed Arithmetic

HT Hough Transform

BP Bit Plane

SIMD Single Instruction Multiple Data

LUT Look-up Table 


\section{List of Publications:}

- Journal papers:

[1] Xiaoxiao Zhang, Sylvain Leomant, Ka Lai Lau and Amine Bermak "A Compact Digital Pixel Sensor (DPS) Using 2T-DRAM", IEEE Journal of Low Power Electronics and Applications, Appl. 2011, 1(1), Page(s): 7796.(Accepted)

[2] Xiaoxiao Zhang, Amine Bermak, Farid Boussaid, "A 32×32-bit Multiprecision Razor-based Dynamic Voltage Scaling Multiplier with Operands Scheduler", IEEE Transaction on Very Large Scale Integration System (Submitted)

[3] Xiaoxiao Zhang, Amine Bermak, Farid Boussaid, Fakhreddine Ababsa, "Bit-Plane Based Local-voting Early-stop Hough Transform Processing for Real-Time Image Segmentation", Electronics Letters (Submitted)

- Refereed international conference papers:

[1] Xiaoxiao Zhang, Amine Bermak, and Farid Boussaid, "Dynamic Voltage and Frequency Scaling for Low-power Multi-precision Reconfigurable Multiplier", IEEE International Symposium on Circuits and Systems, 2010 (ISCAS 2010), Page(s): 45-48, May. 2010.

[2] Xiaoxiao Zhang, Amine Bermak, and Farid Boussaid, "Power Optimization in Multipliers using multi-precision combined with voltage scaling techniques", Quality Electronic Design, 2009. ASQED 2009, Page(s): 79-82, July 2009.

[3] Irene Zheng, Xiaoxiao Zhang, Farid Flitti and Amine Bermak, "Cascaded Classification For Hardware Face Detection", IEEE International Con- 
ference on Electron Devices and Solid-State Circuit 2008 (EDSSC 2008), Page(s): 1-4, Dec, 2008.

[4] Xiaoxiao Zhang, Amine Bermak, Farid Boussaid, and Bouzerdoum, A, "A Compact CMOS Face Detection Architecture Based on Shunting Inhibitory Convolutional Neural Networks", Electronic Design, Test and Applications, 2008 (DELTA 2008), Page(s): 374-377, Jan, 2008. 


\section{Chapter 1}

\section{Introduction}

\subsection{Background and Motivation}

Recent advances in semiconductor fabrication technology have enabled the concept of a single camera-on-a-chip (commonly referred to as CMOS imager), which integrates all camera functions onto a single piece of silicon [1, 2]. An increasingly large number of high volume consumer imaging products do now integrate CMOS imagers. Examples include cell phones, cameras, fax machines, scanners to name a few. The micro-scale camera-on-a-chip enables new applications such as the "pill camera" [3]. This encapsulated miniaturized camera that the patient swallows, passes through the digestive tract, transmitting images of the entire gastrointestinal tract to an external portable recording device. The pill camera has generated widespread interest from the medical community as a means to successfully locate small bowel abnormalities or carry out localized drug delivery in the body [3]. The selling point behind the success of CMOS image sensors lies in the use of the well-established semiconductor industry standard CMOS process, which results in reduced development and fabrication costs for CMOS image sensors. With the advent of deep sub-micron CMOS processes, which feature a minimum lithographic feature size below 0.18 um, it becomes now possible to build high performance single chip cameras, integrating image capture and advanced on-chip processing circuitry [2]. The fully integrated camera-on-chip, 
promises to offer significant advantages in terms of manufacturing cost, system volume and weight, power dissipation and increased built-in functionalities [2]. Another key advantage of CMOS imagers is their high speed capability, with a $352 \times 288$ CMOS digital pixel imager achieving 10,000 frames/s or 1 Giga-pixel/s already demonstrated [4]. The high speed imaging capability of CMOS image sensors can be used to further improve the sensor performance by [2]: (i) extending the sensor dynamic range, (ii) implementing early vision tasks such as tracking or pattern recognition, (iii) estimating motion vectors for tasks such as noise correction, video compression, super-resolution, or motion compensation.

The consistent paradigm in machine vision has been that a "camera" sees the world and a computer "algorithm" recognizes the object [5]. This separation behind sensing and processing, has resulted in bulky and expensive camera systems, exhibiting low latency processing and limited or no sensory adaptation.

CMOS camera-on-a-chip technology has the potential to overcome the aforementioned limitations by offering high speed imaging and allowing extensive processing at the level of the sensing element. Pixel level processing would result in a significantly higher overall processing power and allow pixel-level sensory adaptation to varying illumination conditions.

To enable the concept of a low cost high performance miniature camera-ona-chip with the aforementioned characteristics, the proposed research will aim at developing advanced real-time digital image processing (DIP) cores or modules that could be integrated together with the photo-sensing pixel array. The targeted DIP cores/modules will address the stringent requirements of compactness and low-power real-time operation to enable their integration into a variety of low cost portable consumer imaging products. 


\subsection{Statement of Research Objectives and Con- tribution}

This research is a contribution to the field of CMOS camera-on-a-chip technology with the development of low-power real-time digital image processing cores suitable for on-chip integration. The ability to integrate image capture and advanced processing circuitry on a single low cost chip, would enable the large-scale deployment of these miniature cameras for a number of commercially and socially attractive applications such as homeland security, military operations, remote surveillance and monitoring, smart environments to biomedical implants such as the pill-camera. The range of applications for this technology is increasingly widening as we enter a new age in which electronic devices will be equipped with their own "intelligent eyes" [2]. The proposed research is expected to:

1. Provide the computer vision community with new architectural and computational solutions to enable integration of advanced digital image processing cores inside a camera-on-a-chip.

2. Develop the lowest-power arithmetic units (e.g. multipliers) for a variety of performance targets. Once developed, the power and delay characteristics of these designs enable one to determine the fundamentals limits of each digital image processing core.

3. Develop a novel image sensor architecture that filters data and only transmits information-rich data to allow for rapid data analysis and decisionmaking. To enable low-power DIP system, the sensor should have small pixel area and power dissipation.

4. Develop new and innovative ways of performing computationally extensive on-chip image processing using robust and energy-efficient digital timing circuitry.

5. Develop general guidelines for the development of digital image processing DIP cores suitable for integration in a CMOS camera-on-a-chip. These 
guidelines would be applicable to all three design approaches (custom, semicustom, and synthesized).

\subsection{Thesis Organization}

This thesis is organized as follows:

Chapter 2 introduces the methodologies for developing a low power multiplier unit. Multi-precision architecture, Razor flip-flops based Dynamic Voltage Scaling (DVS) technique, and parallel processing scheme are integrated to obtain great flexibility, and both high performance and minimum power dissipation.

Chapter 3 introduces the operation and implementation of an operands scheduler for our multi-precision DVS multiplier. The operands scheduler is responsible for rearranging the input operands to an optimal sequence that can accelerate the processing speed and further reduce overall power consumption.

Chapter 4 proposes a new DPS architecture based on a multi-reset integration scheme that reduces the sensor area, power and memory requirements. This chapter presents the proposed multi-reset integration scheme and its implementation with dedicated memory circuits. The proposed architecture is validated by a prototype chip fabricated using AMS $0.35 \mu \mathrm{m}$ CMOS technology. Reported experimental results are compared with prior works.

Chapter 5 introduces the algorithm optimization and hardware implementation of a new image segmentation approach - bit-plane based early-stop Hough transform for real time line detection. Our algorithm successfully reduces the computational complexity and the memory requirements of traditional Hough algorithms. We show that the performance of our proposed solution, using FPGA experimental results. It is shown that the proposed implementation remains superior to other methods in terms of both performance and design cost metrics.

Finally, Chapter 6 draws the conclusion and outlines possible future works. 


\section{Chapter 2}

\section{Optimization of Multiplier for Low-Power DIP Cores}

\section{$2.1 \quad$ Introduction}

Given the ever increasing processing power of portable electronic devices and the relatively slower advances in battery lifetime, reducing the power consumption has become critical in VLSI designs [6]. This is particularly true for the multiplier as it probably represents the most essential arithmetic building block in many applications such as DSPs and multimedia devices [7]. Many digital signal processing operations require an extensive amount of multiplications such as convolution, filtering, DCT, vector quantization [10], to name few. In addition, the multiplier typically presents relatively longer latency and larger bit-width, which further makes it nearly the most power-consuming unit.

Recent works have put significant efforts to decrease the multipliers power consumption to enable its integration into battery powered portable systems. A nice property in most sensor applications is, the actual inputs do not always occupy the entire magnitude of its wordlength, and they often vary dramatically. For example, in artificial neural network applications, the weight precision used during the learning phase is approximately twice that of the retrieval phase [8]. Besides, the operations in lower precisions are the most frequently required. Un- 
fortunately, today's most full-custom DSPs and ASICs are mostly designed for fixed maximum wordlength, which aims to accommodate the worst case scenario. In such circumstances, a great part of unused hardware resources will usually produce additional power consumption. For example, an 8-bit multiplication computed on a 32-bit Booth multiplier will lead to serious power inefficiencies due to unnecessary signal switching.

In addition, to enhance the efficiency and minimize the power consumption of the system, the optimal operating point including clock frequency and supply voltage have to be found. Dynamic voltage scaling (DVS) is an effective approach for saving power by adjusting the supply voltage according to circuit's run-time workload instead of fixing the supply voltage to the worst case level [9]. Although some works $[10,11]$ have researched voltage scaling problems, the proposed architectures are restricted to limited number of offline simulated precision-voltage pairs, which is neither flexible for a variety of application requirements nor runtime reconfigurable for precise voltage tuning. To the best of our knowledge, our work constitutes the first attempt to combine multi-precision with DVS technique in multipliers to obtain full reconfigurable ability, and both high performance and low power dissipation.

\subsection{Wordlength and Voltage Optimization Overview}

Several works have researched the wordlength optimization problems. [12, 13] proposed an ensemble of multipliers of different precisions each of which are optimized to a particular scenario. Each pair of incoming operands will be routed to the smallest multiplier that can compute the result to take advantage of the lower energy consumption of the smaller circuit. The ensemble of point systems are reported to consume less power on a series of applications, however, chip area is increased largely because of the ensemble. To address this issue, $[14,15]$ proposed to share and reuse some functional modules within the ensemble. In [14], a 8-bit multiplier is reused for the 16-bit multiplication, adding scalability without 
large area penalty. [15] extended this method by making pipelining to further improve the multiplier's performance. A more flexible approach is realized by [16], which groups multiplier elements together to provide higher precisions with reconfigurability feature for efficient use of hardware resources. [17] analyzed the overhead problem of such kind of reconfigurable multipliers, around 10-20\% of extra chip area and corresponding power consumption are needed for 8-16 bits multipliers. In the aforementioned multipliers, the non-monolithic precision results in: (i) non-negligible silicon area and power overhead; (ii) performance and throughput reduction brought by the shutdown of parts of the circuit; (iii) great margins to the operating condition (eg. voltage and operating frequency) of the multiplier.

When adjusting the supply voltage, the actual performance of the multiplier running under scaled voltage has to be characterized to guarantee a fail-safe operation. Conventional DVS techniques are mainly LUT based approach and on-chip critical path replica approach $[18,19,20]$. LUT approach tunes voltage according to a pre-defined voltage-frequency relationship stored in a LUT, which is determined at the combination of worst case conditions (process variations, power supply droops, temperature hot-spots, coupling noise, etc). As a result, big margins are necessarily added, which significantly decrease the efficiency of the DVS technique. Critical path replica approach usually uses an on-chip critical path replica to approximate the actual critical path. The supply voltage could be scaled to the extent that the replica fails to meet the timing. However, safety margins for compensating the intra-die delay mismatches and addressing the fastchanging transient effects are still needed. Most importantly, the critical path may change with the changing supply voltage. Even at a fixed supply voltage, the critical path may change due to process and temperature variations. If this occurs, operations would completely fail even when considering all safety margins.

The aforementioned disadvantages motivate recent research efforts the socalled "error-tolerant" approaches [21, 22, 23]. Error-tolerant techniques can operate the circuit at run-time even at a voltage level for which timing errors occur. A recovery mechanism is then applied to restore the correct data. Due 
to the removal of the worst-case margin and safety margins, this approach significantly improves the efficiency of the DVS technique. Our work adopts this "error-tolerant" approach and uses razor flip-flops [21] to determine the minimum voltage.

\subsection{Design of Multi-precision DVS Low-Power Multiplier}

\subsubsection{System Overview}

To demonstrate our highly flexible architecture and minimum energy design methodology, we propose a low power reconfigurable multiplier architecture that combines multi-precision with parallel processing approach to make the most efficient use of the hardware resources, and simultaneously incorporates razor-based DVS technique to significantly reduce the power consumption. Conventionally, these approaches would result in: (i) non-negligible silicon area and power overhead due to reconfigurability, (ii) large silicon area and power overhead due to DVS and DFS units, (iii) performance and throughput reduction brought by the shut-down of parts of the circuit and/or the use of reduced supply voltage. Trading throughput for power cannot achieve high performance and low power by adapting its structure to computational requirements over time. In this work, we address these limitations by dedicated optimizations from system, to algorithm, to logic levels. Besides, because parallel processing enables our multiplier behave like a SIMD unit, we designed a dedicated operands scheduler that rearranges the operands to an optimal sequence, that accelerates the processing speed, reduces the transitions among different voltage and frequency levels, and minimizes the overall power consumption. This enables the multiplier to run-time adapt to the actual workload of the targeted application by automatically operating at the minimum possible level of supply voltage and clock frequency, which results in a significant power reduction while having no degradation on the applications' performance/throughput requirements. 
Fig.2.1 depicts the multiplier system architecture. The multi-precision multiplier uses four different modules: (i) the input operands scheduler (IOS) whose function is to reorder the input data stream into a buffer so as to reduce the required power supply voltage transitions; (ii) the frequency scaling unit (FSU) implemented using a voltage controlled oscillator (VCO). Its function is to generate the required operating frequency of the multiplier; (iii) the voltage scaling unit (VSU) implemented using voltage dithering technique to limit silicon area overhead. Its function is to dynamically generate the supply voltage so as to minimize power consumption; and (iv) the dynamic voltage/frequency management unit (VFMU) which receives the user requirements (e.g throughput). The VFMU sends control signals to the VSU and FSU to generate the required power supply voltage and clock frequency for the multi-precision multiplier.

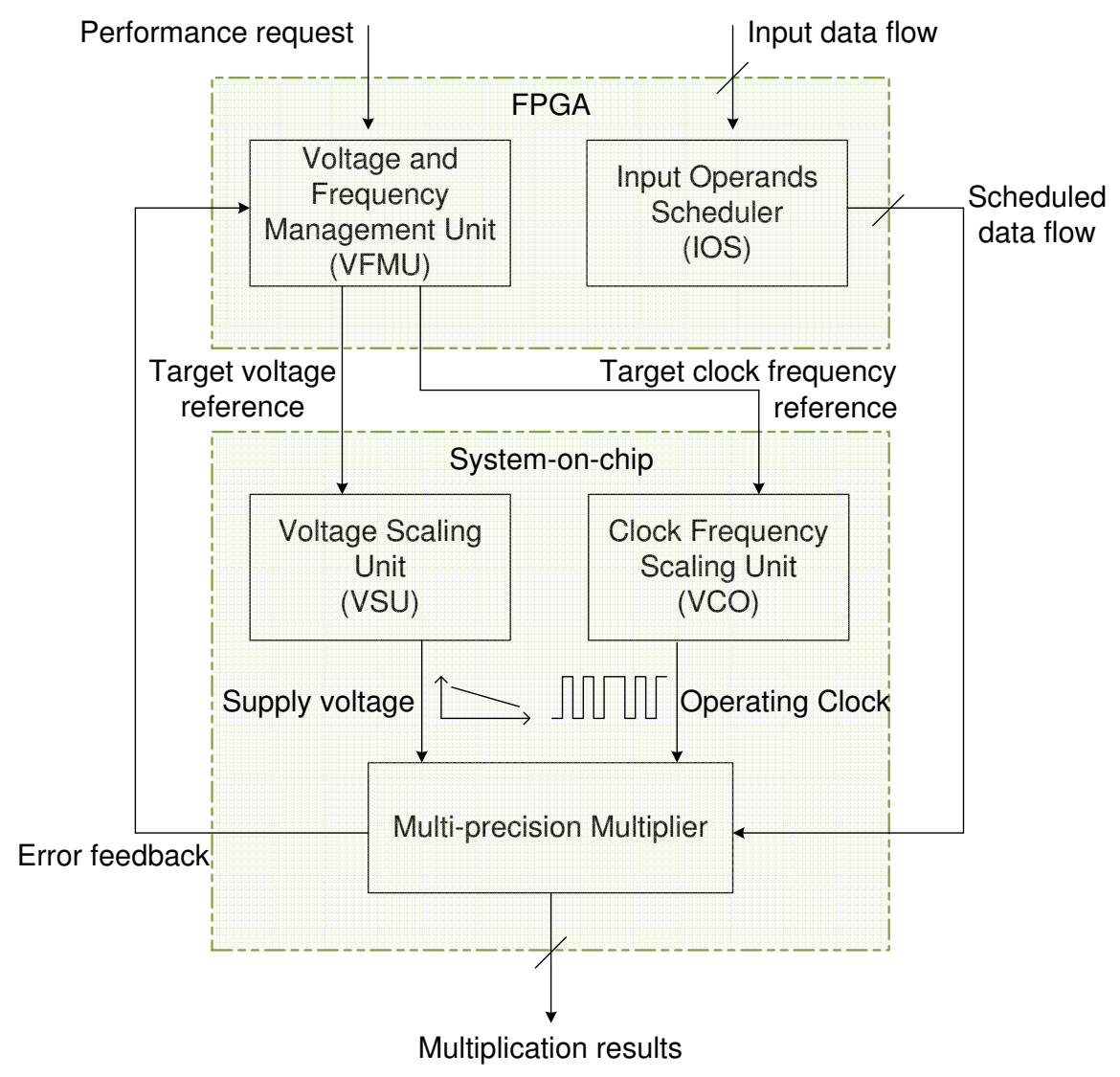

Figure 2.1: Multiplier system architecture.

The multi-precision multiplier is responsible for all computations. It is equipped with razor flip-flops, which can report timing errors associated to insufficiently high voltage supply levels. The operation principle is as follows. Initially, 
the multiplier operates at a standard supply voltage of $3.3 \mathrm{~V}$. If the razor flip-flops of the multiplier continue reporting no errors, this means that the supply voltage can be reduced. The VFMU will send control signals to the VSU to tune the supply voltage down. When the feedback provided by the razor flip-flops indicate timing errors, the scaling of the power supply is stopped. The principle behind the proposed multi-precision/DVS architecture is that, the actual multiplication time required for a short-length operand is much shorter than that of the allocated time interval. This idling time could be exploited by DVS to lower the supply voltage level. Fig.5.2 reports simulation results for delay, minimal supply voltage, and power consumption of different bit-width multipliers operating at $50 \mathrm{MHz}$ clock frequency. We can see that the supply voltage can indeed be drastically reduced when the input bit-width decreases, resulting in significant power consumption savings.

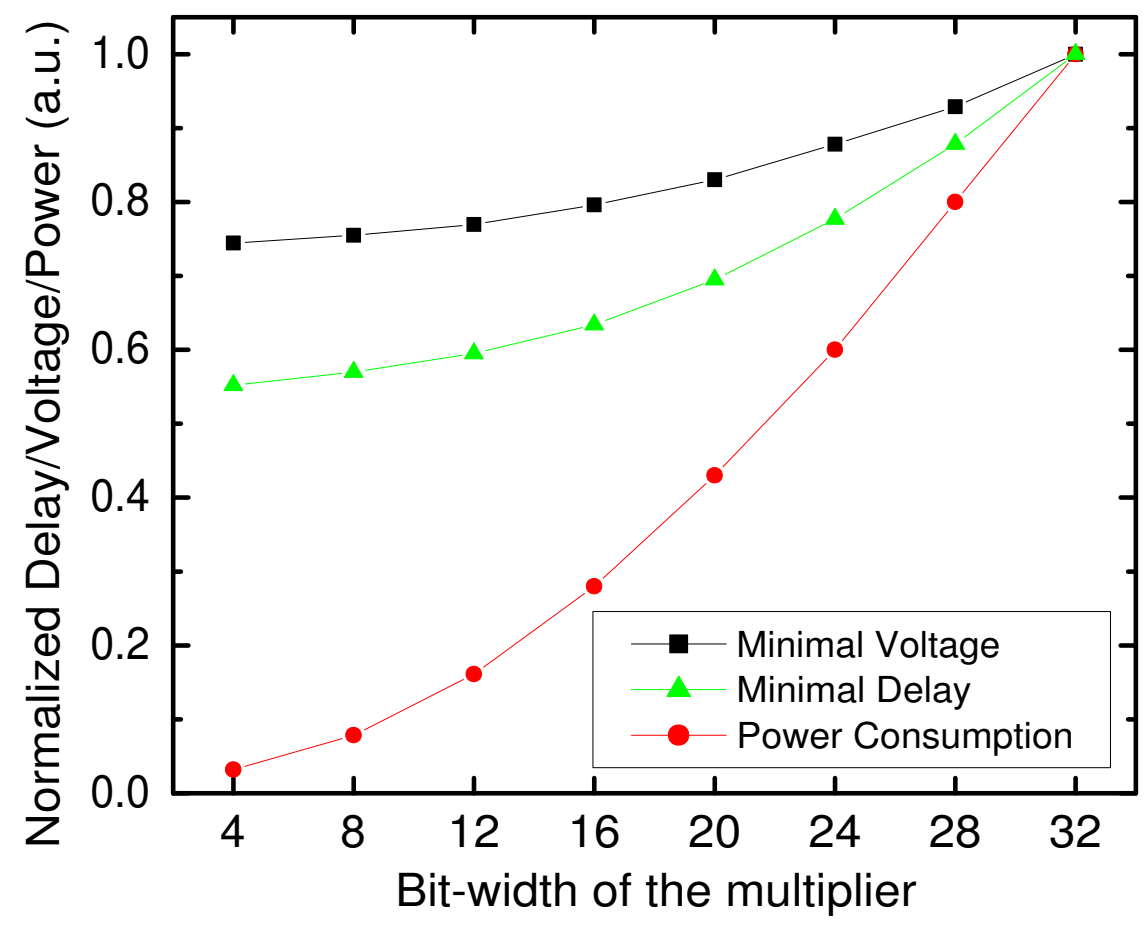

Figure 2.2: Normalized delay, minimal voltage, and power consumption as a function of the bit-width of the multiplier.

In addition to multi-precision and DVS, we propose to combine parallel processing. As shown in Fig.2.3, the proposed multiplier is built using nine 8bit $\times 8$ bit reconfigurable multipliers. These building blocks can either work as 9 independent multipliers or work in parallel to perform one, two or three 16 bit $\times 16$ bit 
multiplications or a single 32 bit $\times 32$ bit operation. Parallel processing can be used to increase the throughput (eg. 4/9 8bit $\times 8$ bit or $2 / 316$ bit $\times 16$ bit multiplications) or reduce the supply voltage level, and hence the power consumption. To explain this, let's assume that the throughput of the maximum-precision mode (32bit $\times 32$ bit) is $T$, and that the operating frequency is $f$. In the 3 parallel 16bit $\times 16$ bit mode, the operating frequency could be reduced to $1 / 3 f$ and to $1 / 9 f$ in the 9 parallel 8 bit $\times 8$ bit mode.

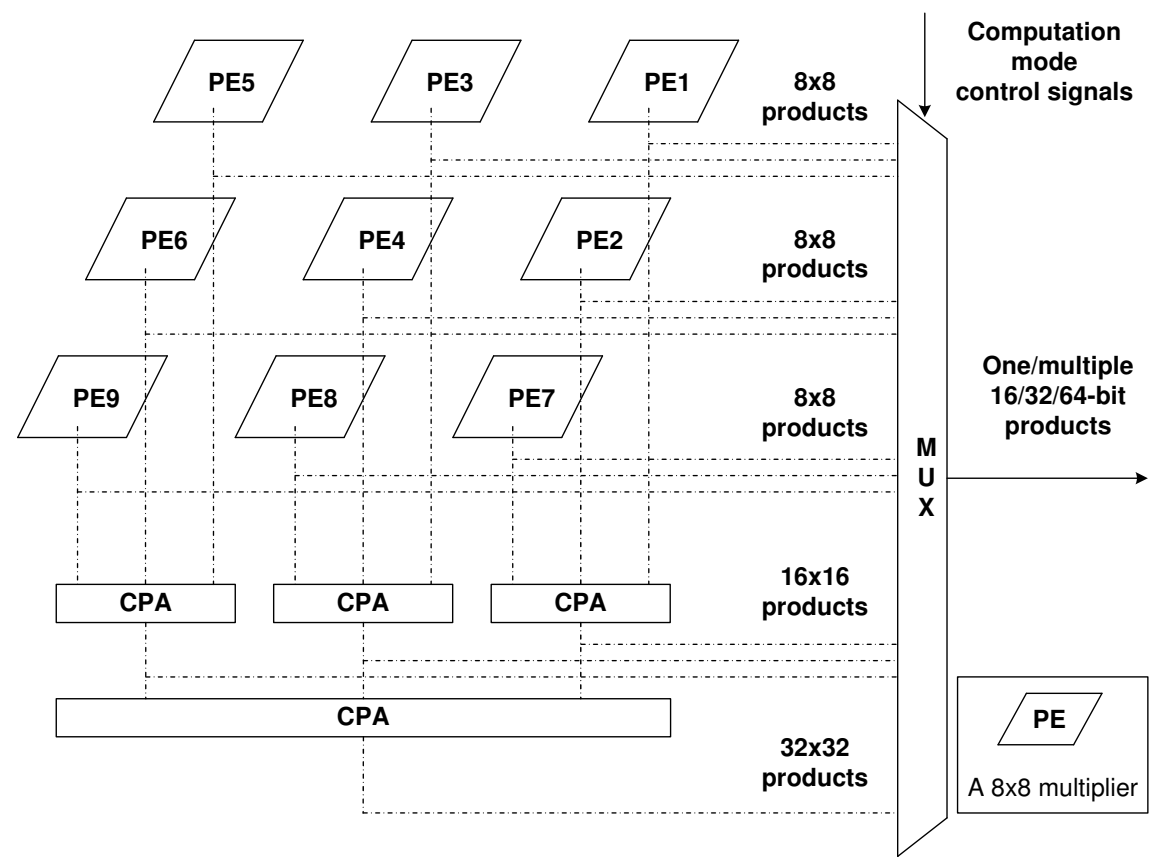

Figure 2.3: Possible configuration modes of the proposed multi-precision multiplier.

Fig.2.4 shows the benefits of the proposed approach in terms of power consumption. Curve 1 corresponds to the case of a fixed-precision multiplier using a fixed power supply. Note that power consumption is a linear function of the workload, which is normally represented by the input operands precision. It is important to point out that, for a given precision, say 16-bit, the power consumption will depend on whether the multiplication is performed using a 16-bit multiplier (curve (2)), or a 32-bit multiplier (curve (1)). This is because the redundant MSBs cause large amount of unnecessary switching, especially in high-speed tree multipliers. The same observations could be made for a 8-bit multiplication (curve (3) vs. curve (1)). In Fig.2.4, region 1 represents the power optimization space for multi-precision techniques, which use different precision multiplications 
to reduce power. If one combines multi-precision with DVS, power is further reduced with curves (1),(2),(3) becoming curves (4),(5),(6) respectively. Regions 1 and 2 show the power optimization space for the combined approach. Note that the low-precision segments (0-8 and 9-16 bits precision) are further downward, but also that the high-precision segments (17-32 bits precision) are also shifted away from the original linear curve. With parallel processing, the operating frequency could be decreased. As result, the supply voltage could further be lowered, as shown by curves (7) and (8) of Fig.2.4. Finally, region 3 represents the optimization space for the proposed approach, which combines multi-precision, DVS with parallel processing.

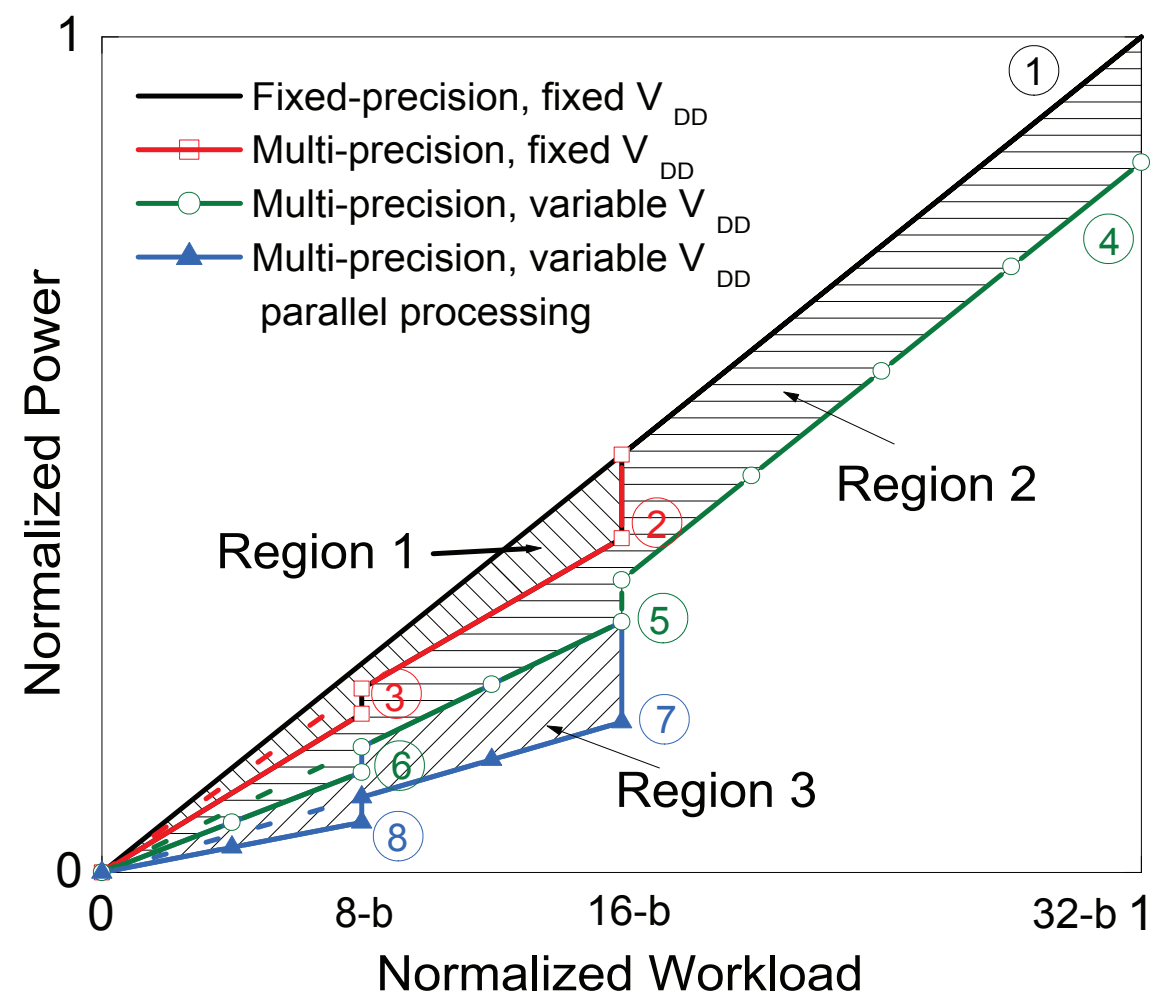

Figure 2.4: Optimization space of multi-precision, DVS, and parallel processing approaches.

\subsubsection{Multi-precision and Reconfigurability Overhead}

Fig.2.5 depicts the structure of the input interface unit used to manipulate the incoming multi-precision operands. An extra MSB sign bit is added to enable both signed and unsigned multiplications. A 3-bit control bus indicates whether the inputs are 1/4/9 pair(s) of 8-bit operands, or 1/2/3 pair(s) of 16-bit operands, 
or 1 pair of 32-bit operands, respectively. Depending on the selected operating mode, the input data stream is distributed (Fig.2.5) between the PEs to perform the computation. Fig.2.6 shows how three 8 bit $\times 8$ bit processing elements (PEs) are used to realize a 16 bit $\times 16$ bit multiplier. The 32 bit $\times 32$ bit multiplier is constructed using a similar approach but requires $3 \times 3$ PEs. A 3bit-control word defines which PEs work concurrently and which PEs are disabled. Whenever the full precision (32bit $\times 32$ bit) is not exercised, the supply voltage and the clock frequency may be scaled down according to the actual workload.

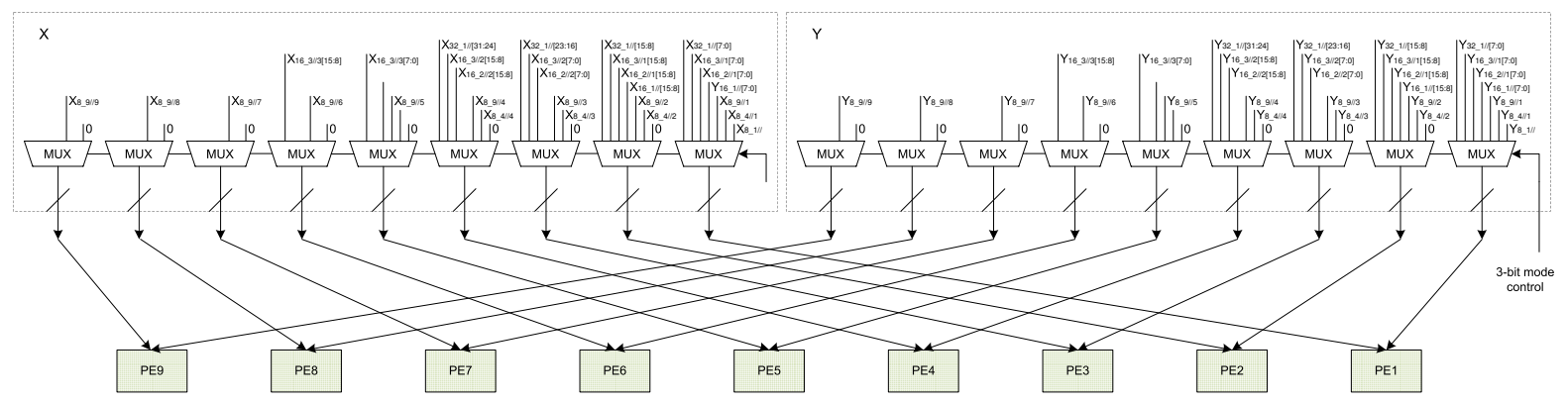

Figure 2.5: Structure of the input interface unit.

To evaluate the overhead associated to reconfigurability and multi-precision, we define $X$ and $Y$ as the 2n-bits wide multiplicand and multiplier, respectively. $X_{H}, Y_{H}$ refer to their respective $n$ most significant bits while $X_{L}, Y_{L}$ refer to their respective $n$ least significant bits. $X_{L} Y_{L}, X_{H} Y_{L}, X_{L} Y_{H}, X_{H} Y_{H}$ represent crosswise products. The product of $X$ and $Y$ can be expressed as:

$$
P=\left(X_{H} Y_{H}\right) 2^{2 n}+\left(X_{H} Y_{L}+X_{L} Y_{H}\right) 2^{n}+X_{L} Y_{L}
$$

This equation shows that a 2n-bit reconfigurable multiplier can be built using adders and four n-bit $\times$ n-bit multipliers to compute $X_{H} Y_{H}, X_{H} Y_{L}, X_{L} Y_{H}$ and $\left.X_{L} Y_{L}\right)$. Table.2.1 shows that this would result in overheads of $18 \%$ and $13 \%$ for the silicon area and power, respectively. However, if we define [24]: 


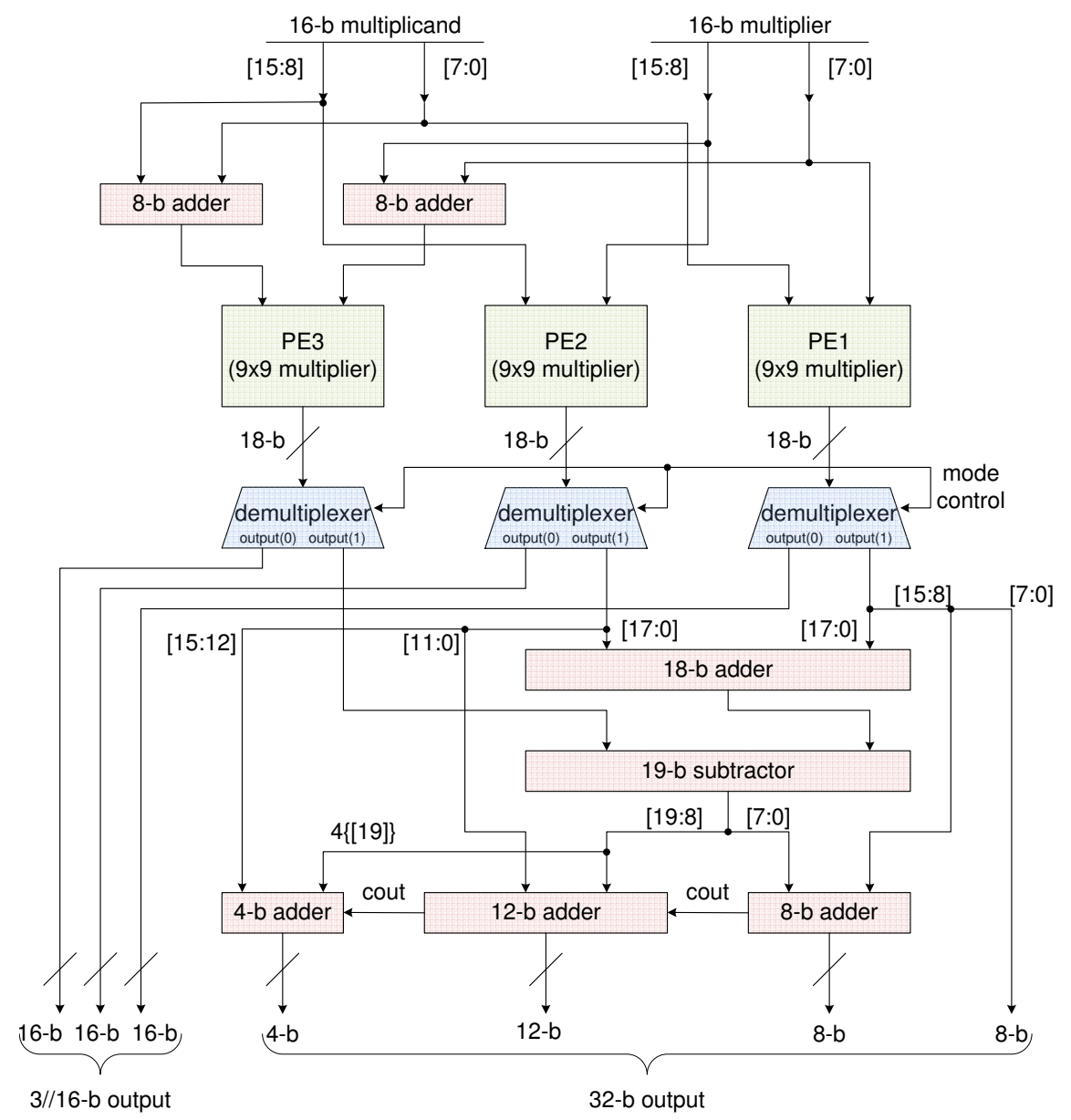

Figure 2.6: How three processing elements (PEs) can be combined to form a 16bit $\times 16$ bit multiplier. 


$$
\begin{array}{r}
X^{\prime}=X_{H}+X_{L} \\
Y^{\prime}=Y_{H}+Y_{L}
\end{array}
$$

then Eq.2.1 could be rewritten as:

$$
P=\left(X_{H} Y_{H}\right) 2^{2 n}+\left(X^{\prime} Y^{\prime}-X_{H} Y_{H}-X_{L} Y_{L}\right) 2^{n}+X_{L} Y_{L}
$$

Comparing equations (2.1) and (2.4), one can note that we have removed one n-bit $\times$ n-bit multiplier (for calculating $X_{H} Y_{L}$ or $X_{L} Y_{H}$ ) and one 2n-bit adder (for calculating $X_{H} Y_{L}+X_{L} Y_{H}$ ). The two adders have been replaced them with two n-bit adders (for calculating $X_{H}+X_{L}$ and $Y_{H}+Y_{L}$ ) and two $(2 \mathrm{n}+2)$-bit subtractors (for calculating $X^{\prime} Y^{\prime}-X_{H} Y_{H}-X_{L} Y_{L}$ ). In the case of a 32-bit multiplier, we can thus significantly reduce the design complexity by using two 34 -bit subtractors to replace a 16 bit $\times 16$ bit multiplier. Note that here we actually need two 16bit $\times 16$ bit multipliers (for calculating $X_{H} Y_{H}$ and $X_{L} Y_{L}$ ) and one 17 bit $\times 17$ bit multiplier (for calculating $X^{\prime} Y^{\prime}$ ). Table 2.1 shows that the 3 subblock architecture can achieve reductions of $16 \%$ in power and $28 \%$ in area as compared to a fixed-width multiplier design.

Table 2.1: Area and Power Comparison of the proposed multi-precision multipliers and traditional fixed-width Multipliers running at $50 \mathrm{MHz}$

\begin{tabular}{c|c|c|}
\hline Schemes & power $(\mathrm{mW})$ & area $\left(\mathrm{mm}^{2}\right)$ \\
\hline \hline 32-bit & 39.62 & 0.624 \\
Fixed-width Multiplier & $(100 \%)$ & $(100 \%)$ \\
\hline 32-bit 4 sub-block & 44.76 & 0.736 \\
Multi-precision Multiplier & $(113 \%)$ & $(118 \%)$ \\
\hline 32-bit 3 sub-block & 33.36 & 0.448 \\
Multi-precision Multiplier & $(84 \%)$ & $(72 \%)$ \\
\hline
\end{tabular}

A conventional 32-bit fixed-width multiplier uses a Booth radix-4 wallace tree structure similar to that used in designing the building blocks of our multiprecision multipliers. However, because of its larger size, a 32-bit fixed-width multiplier exhibits an irregular layout with complex interconnects. This limitation of tree multipliers happens to be addressed by our multi-precision 32-bit 
multiplier, which uses a more regular design to partition, regroup and sum partial products.

\subsubsection{Dynamic Voltage and Frequency Scaling Manage- ment}

In our implementation (Fig.2.1), a dynamic power supply and a voltage controlled oscillator (VCO) are employed to achieve real-time dynamic voltage and frequency scaling under different operation conditions. As discussed in [25], nearoptimal dynamic voltage scaling can be achieved when using voltage dithering, which exhibits faster response time than conventional voltage regulators. Voltage dithering uses using power switches to connect different supply voltages to the load, depending on the time slots as shown in Fig.2.7. In that case, an intermediate average voltage can be achieved. In fact, the conventional voltage dithering technique has some limitations. If the switches are toggled with overlapping periods, both MP1 and MP2 can be turned on at the same time, giving rise to a large transient current from VDDH to VDDL. Fig.2.8 shows the effect of this large leakage when the gate control for MP1 and MP2 are implemented as shown in Fig.2.7. Notice that the current through MP2 is significant at the falling edge of the clock signal due to the inverter. To address this issue, non-overlapping clocks could be used to control MP1 and MP2 (Fig.2.7). Yet, this may result in system instability as there are instances where all supply voltages are disconnected from the load. The requirement for multiple supplies can also result in system overhead.

In order to address these issues, we implemented a single supply voltage dithering scheme (Fig.2.9a), which operates as follows. When the supply voltage $(\mathrm{Vn})$ of the multiplier drops below the predefined reference voltage (Vref), the comparator output (Va) toggles. As a result, the voltage and frequency management unit (VFMU) turn on the power switch via Vetrl, for a predefined duration Tc. The duration Tc and the storage capacitor Cs are selected so that the ripple voltage at the node $\mathrm{Vn}$ is approximately $50 \mathrm{mV}$. Fig.2.9(b) shows the experimental results of the voltage control loop. 


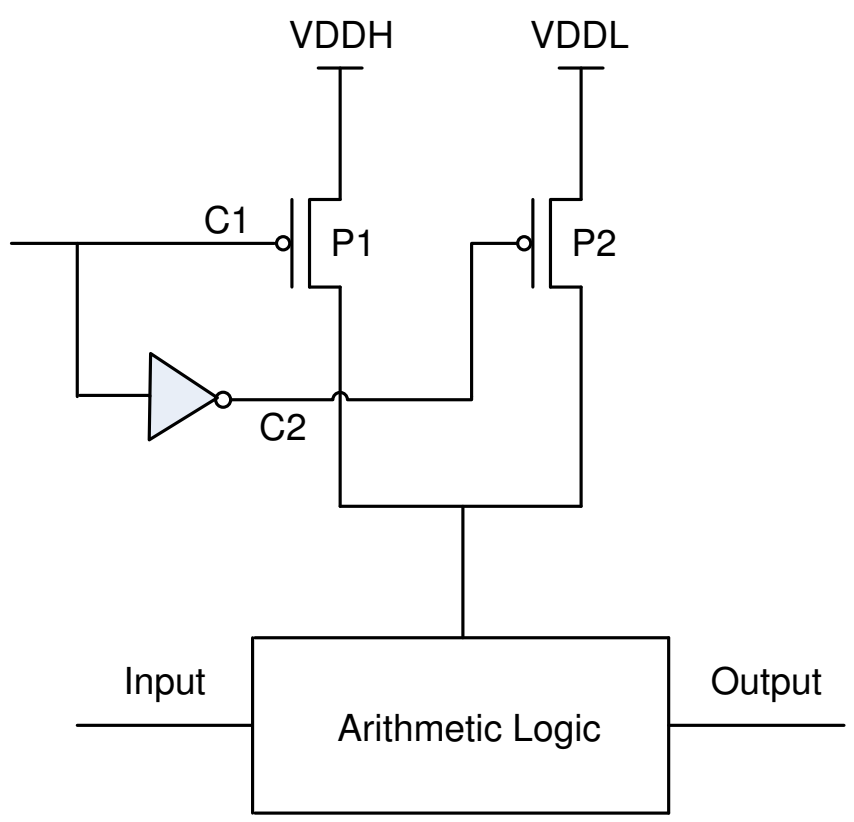

Figure 2.7: DVS arithmetic logic using two header switches for voltage dithering [23].
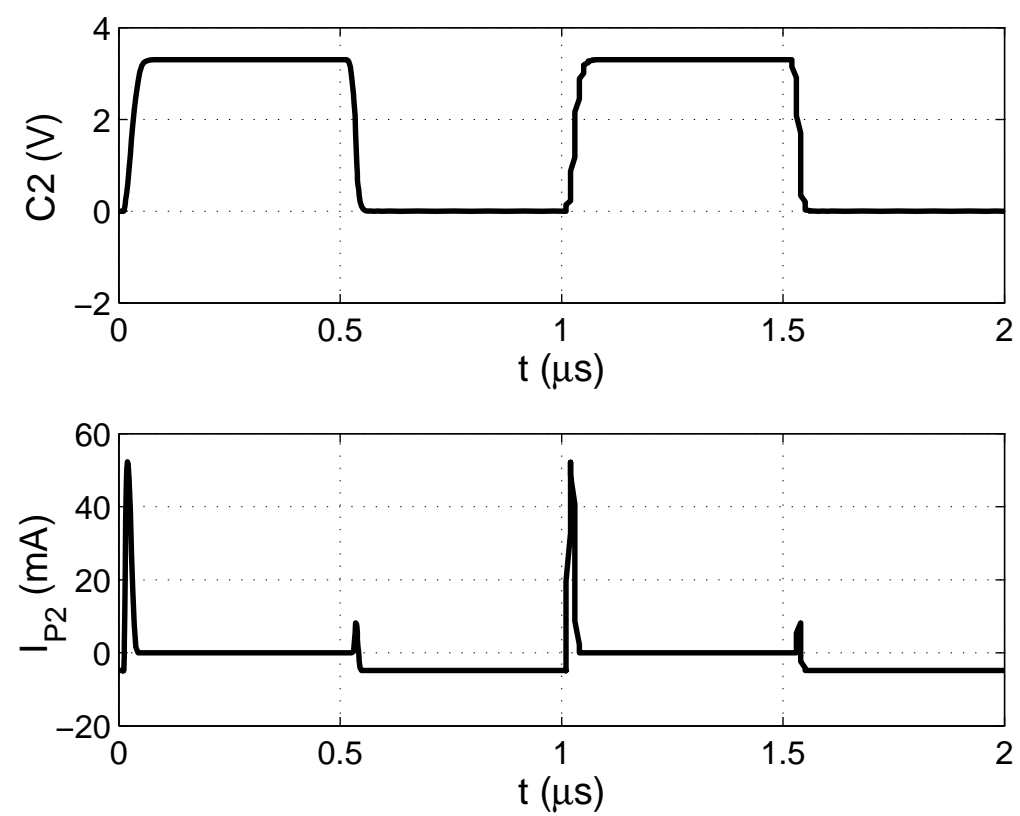

Figure 2.8: Transient Vdd switching current. 


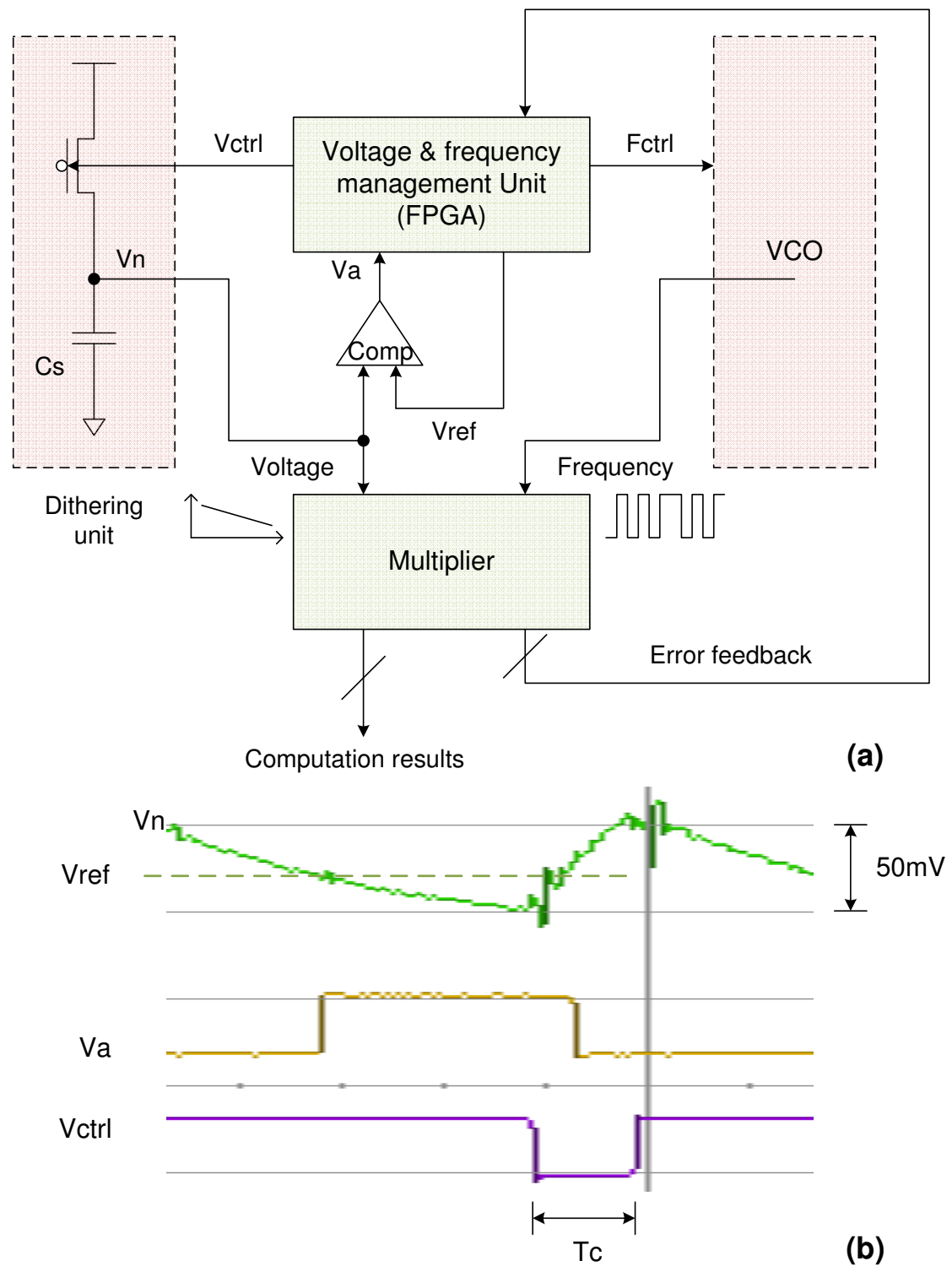

Figure 2.9: (a) The proposed single-header voltage dithering unit and the voltage and frequency tuning loops. (b) Experimental results of timing of the voltage dithering unit. 
In the proposed 32-bit multi-precision multiplier, dynamic frequency tuning is used to meet throughput requirements. It is based on a voltage controlled oscillator (VCO) implemented as a 7-stage current starved ring oscillator. The VCO output frequency can be tuned from 5 to $50 \mathrm{MHz}$ using 4 control bits $(5 \mathrm{MHz}$ per step), as shown in Fig.2.10. Within this frequency range, the frequency error is at most $\pm 12 \%$ (worst case) when considering different possible process corners. The simulated power consumption for the VCO ranges from $85 \mu W(5 \mathrm{MHz})$ to $149 \mu W(50 \mathrm{MHz})$, which is negligible compared with the power consumed by the multiplier. Fig.2.11 reports experimental measurements showing the transient response for the worst case frequency switching (from $50 \mathrm{MHz}$ to $5 \mathrm{MHz}$ ). Note that the clock frequency can settle within one clock cycle as required.

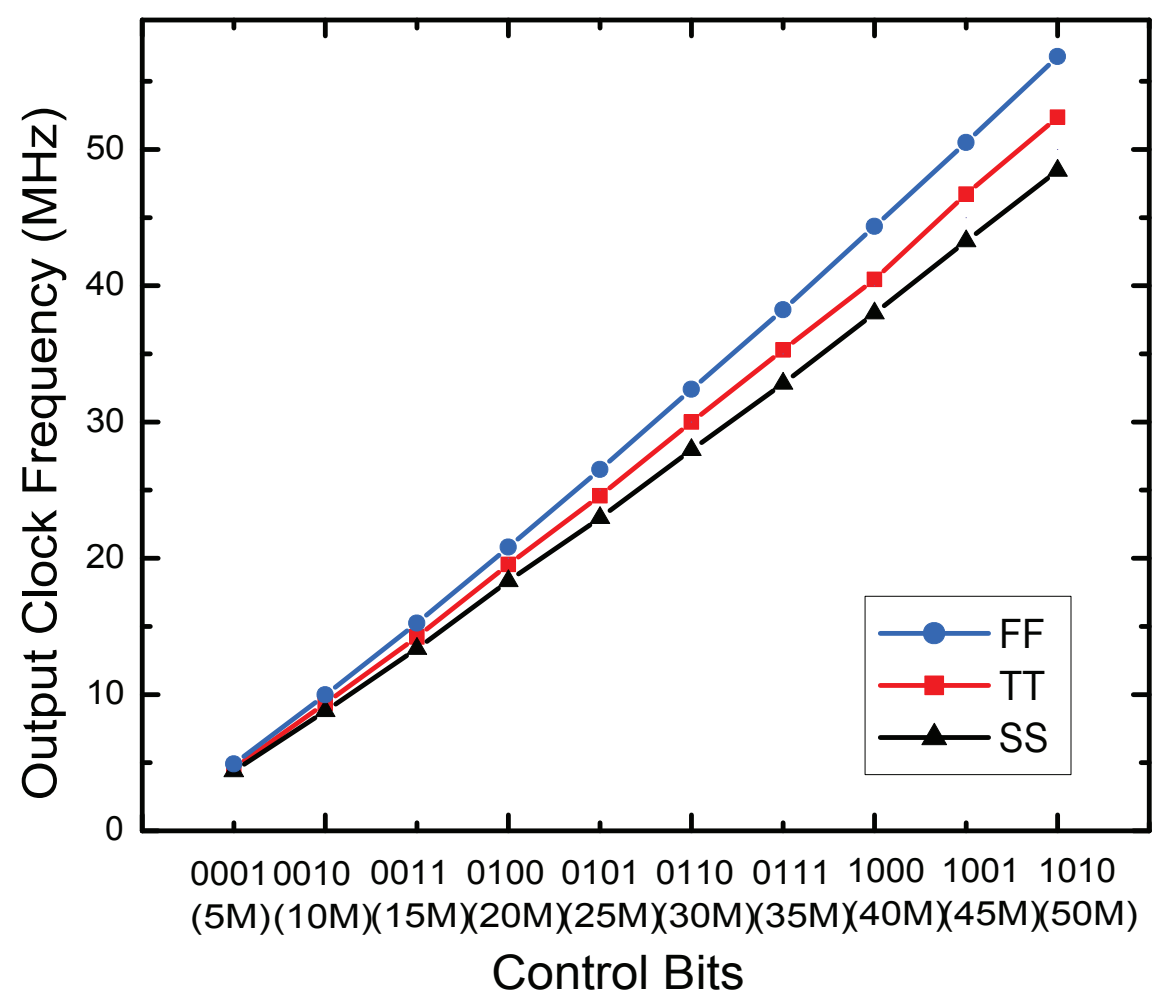

Figure 2.10: Output clock frequency as a function of control bits sequence for different process corners.

\subsubsection{Implementation of Razor Flip-flops}

Although the worst cases are very rarely exercised, traditional DVS approaches still maintain safety margins to ensure proper circuit operation, resulting in excessive power dissipated. The Razor technology [21] is a breakthrough work, which 


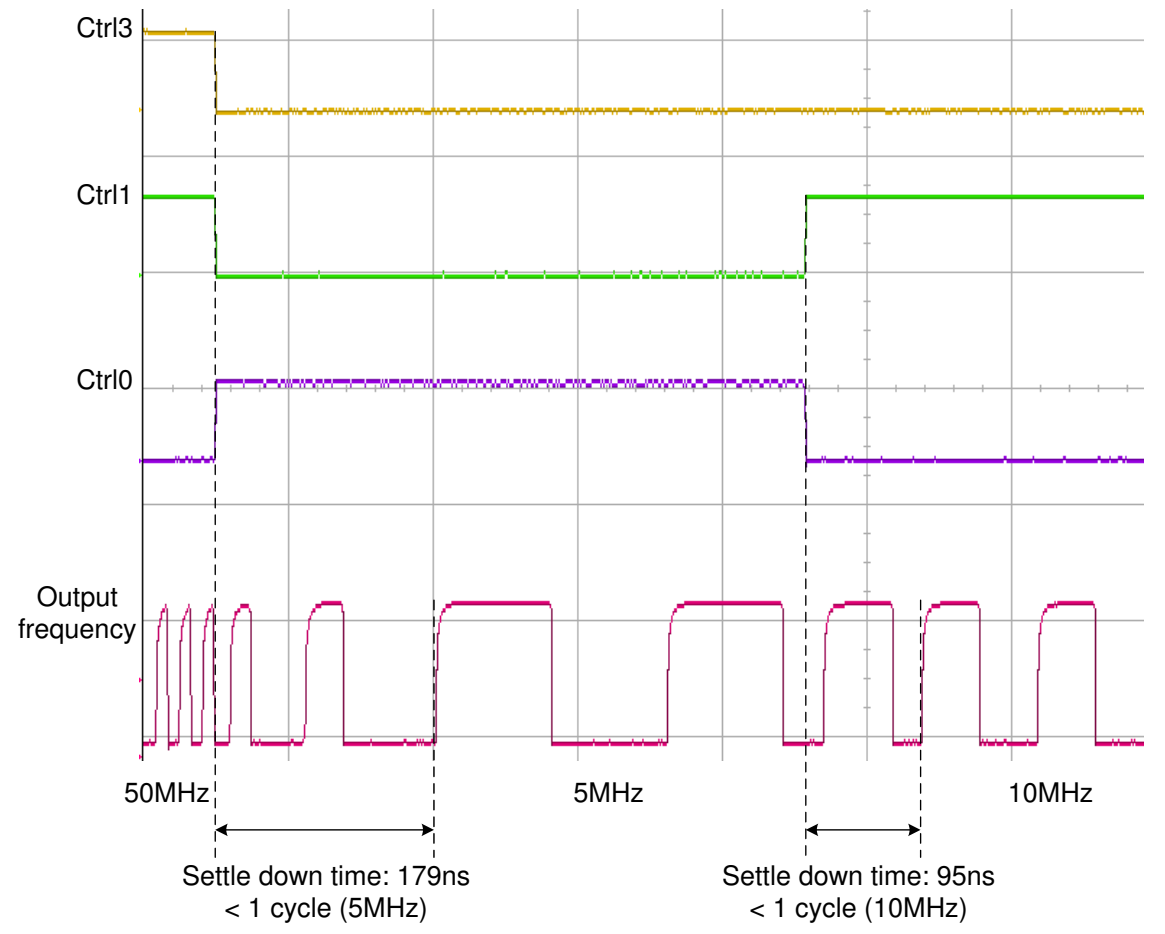

Figure 2.11: Experimental measurement of the worst case frequency switching (from $50 \mathrm{MHz}$ to $5 \mathrm{MHz}$ ).

largely eliminates the safety margins by achieving variable tolerance through insitu timing error detection and correction ability. Power is only spent for the worst or near-worst cases and only when those occur. This approach is based on a razor flip-flop, which detects and corrects delay errors by double sampling (Fig.2.12). The razor flip-flop operates as a standard positive edge triggered flipflops coupled with a shadow latch, which samples at the negative edge. As a result, the input data is given the duration of the positive clock phase to settle down to its correct state before being sampled by the shadow latch. The minimum allowable supply voltage needs to be set such that the shadow latch always clocks the correct data even for the worst case conditions. However, because it is clocked later than the main flip-flop, this requirement is usually satisfied. A comparator flags a timing error when it detects a discrepancy between the speculative data sampled at the main flip-flop and the correct data sampled at the shadow latch. The correct data would subsequently overwrite the incorrect signal.

Since the critical paths of arithmetic units are rarely exercised, the razor flip-flop has a relatively small overhead. Only a few potential critical paths need to be equipped with razor flip-flops. In the absence of a timing error, the razor 


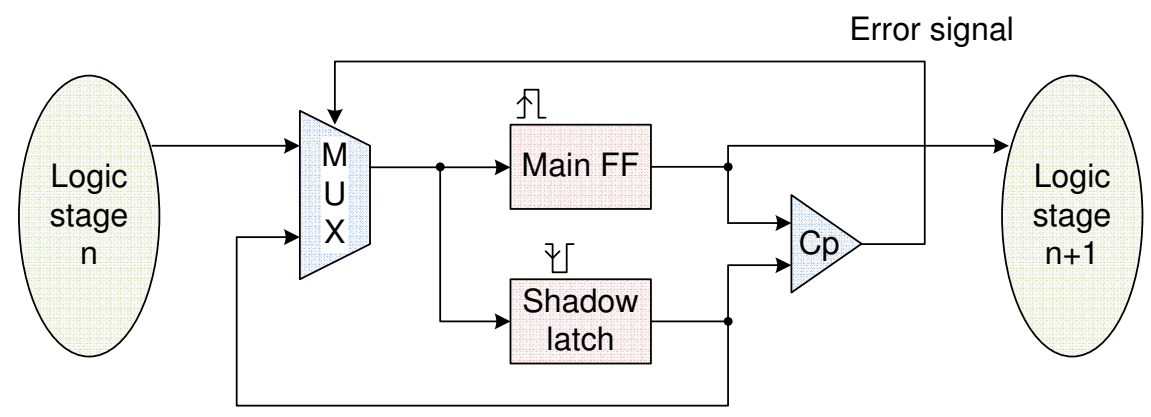

Figure 2.12: Conceptive view of the Razor flip-flop [14].

flip-flop behaves like a standard positive edge triggered flip-flop. There are only rare cases, for which computation fails to meet the timing. In theses cases, the razor flip-flop would consume more power to detect and recover the timing error. At a supply voltage of $3.3 \mathrm{~V}$ and an operating frequency of $50 \mathrm{MHz}$, the razor flipflop was found to consume 1.2 times more static/switching power $(70 \mu \mathrm{W} / 57 \mu \mathrm{W})$ in the case of no timing errors detected. In the other case, it consumes 4.2 times more static/switching power $(239 \mu W / 57 \mu W)$. However, for a conservative activity factor of $1 \%$, the power overhead due to razor flip-flops was estimated to be less than $2.3 \%$ of the nominal chip power because only $9 \%$ of the flip-flops have potential critical paths and thus need to be made razor flip-flops.

\subsection{Performance Evaluation and Discussion}

We designed and fabricated a 32 bit $\times 32$ bit reconfigurable multiplier in AMIS $0.35 \mu \mathrm{m}$ technology. The die photograph of the multiplier system is shown in Fig.2.13 and the implementation characteristics are provided in Table.2.2. The operating mode of the multiplier is controlled by 3 external signals. The operating voltage and frequency is tuned automatically depending on the real workload of the multiplier. The chip was tested by feeding in randomly generated operands and comparing the outputs with the results from a PC, which executed the same data. The 32-bit precision data sets include data with the effective word-length of 17-32 bits. The 16-bit precision data sets and 8-bit precision data sets include data with the effective word-length of 9-16 bits and 0-8 bits respectively. We achieved full functionality across a voltage range of $0.8 \mathrm{~V}$ to $3.3 \mathrm{~V}$, and a frequen- 
cy range of $5 \mathrm{M}$ to $50 \mathrm{M}$.

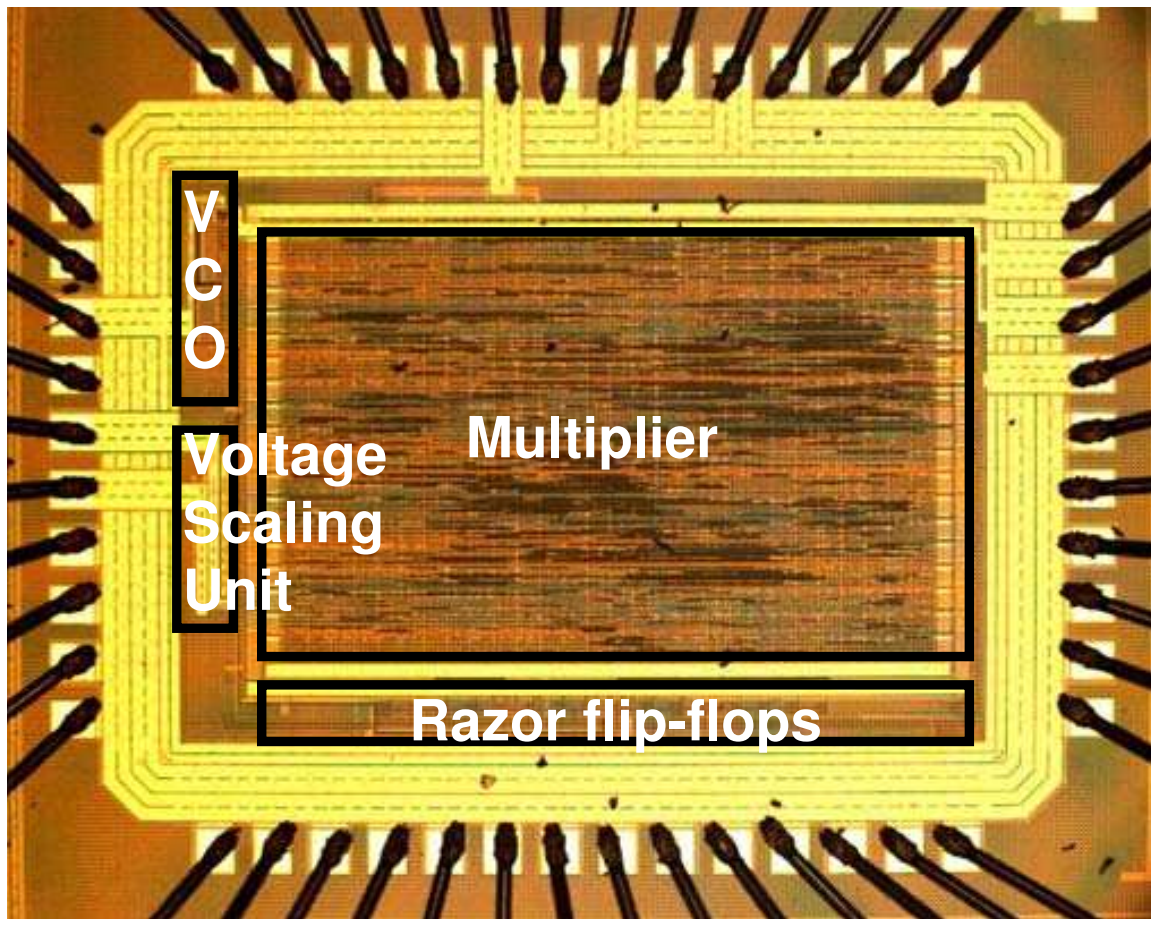

Figure 2.13: Microphotograph of the $32 \times 32$-bit multi-precision multiplier.

Table 2.2: Prototype Characteristics

\begin{tabular}{l|l}
\hline \hline Technology Node & $0.35 \mu \mathrm{m}$ \\
\hline Die Size & $1.5 \mathrm{~mm} \times 1.0 \mathrm{~mm}$ \\
\hline Total Number of Transistors & 37656 \\
\hline Measured Chip Power at 3.3V & $39 \mathrm{~mW}$ \\
\hline DVS Supply Voltage Range & $0-3.3 \mathrm{~V}$ \\
\hline DFS Clock Frequency Range & $5-50 \mathrm{MHz}$ \\
\hline Total Number of Flip-flops & 144 \\
\hline Number of Razor Flip-flops & 13 \\
\hline Standard D Flip-flop Power & $57 \mu W$ \\
\hline $\begin{array}{l}\text { Razor Flip-flop Power } \\
\text { (static/switching) }\end{array}$ & $70 \mu \mathrm{W} / 239 \mu \mathrm{W}$ \\
\hline Total Power Overhead of Razor Flip-flops & $2.3 \%$ \\
\hline \hline
\end{tabular}

Fig.2.14 shows the relation between the minimum supply voltage and operating frequency for different precision modes. As explained previously, razor energy savings are due to both the elimination of safety margins and the processing below the first failure voltage. By scaling the voltage below the first failure point, the error rate of $0.1 \%$ is maintained and the power consumption is measured at this minimum possible voltage. For an operating frequency of $50 \mathrm{MHz}$, the supply voltage is set to $2.45 \mathrm{~V}, 1.95 \mathrm{~V}$ and $1.80 \mathrm{~V}$ for the 32 -bit, 16 -bit, and 
8-bit modes respectively. The whole chip can work across a voltage range of $0.8 \mathrm{~V}$ to $3.3 \mathrm{~V}$. The lower the frequency, the smaller the minimum voltage hence the power consumption.

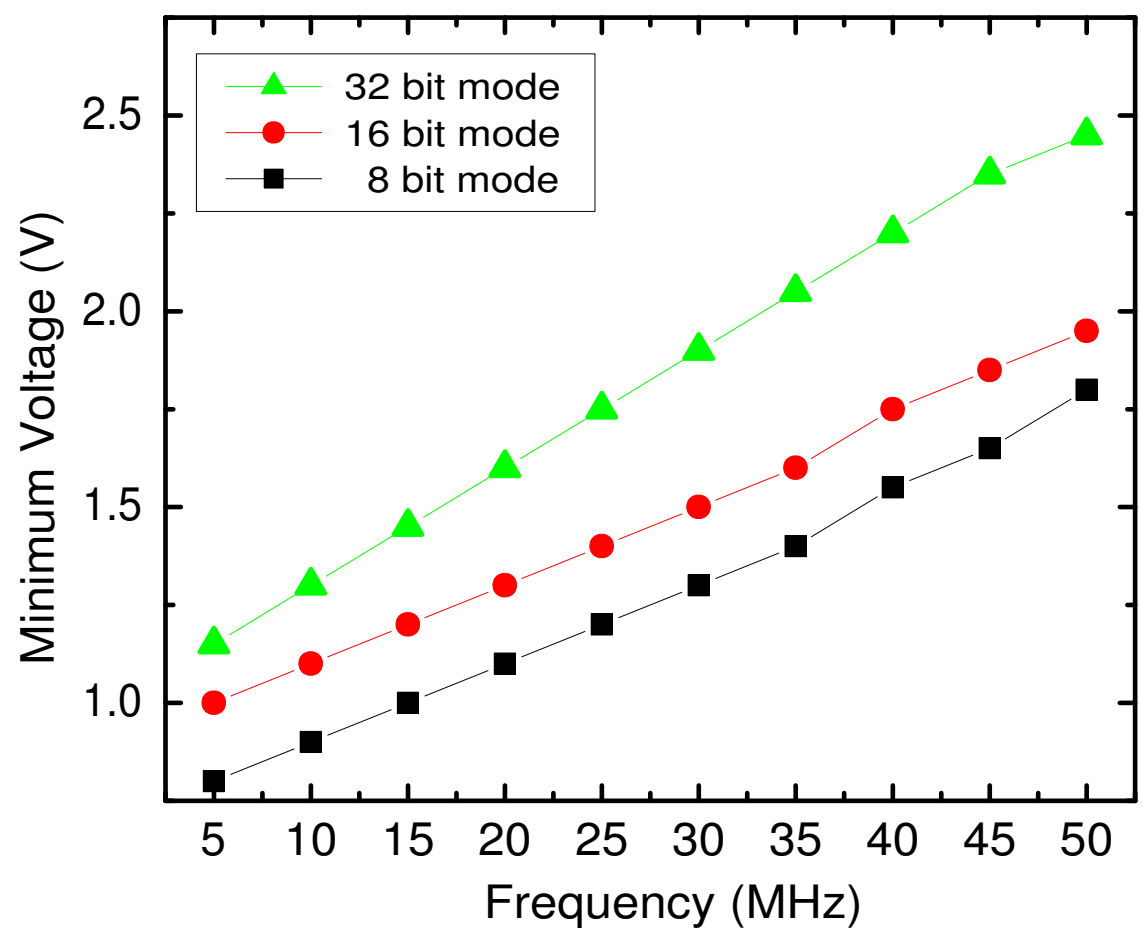

Figure 2.14: Experimental measurements of the minimum voltage supply for different precisions and operating frequencies.

Fig.2.15 reports the chip power consumption for different operating modes. For 16 -bit operands, $55.6 \%(17.35 \mathrm{~mW}$ vs. $39.04 \mathrm{~mW})$ power reduction can be obtained by the multi-precision (MP) scheme. After the DVS technique is applied, the chip consumes $6.06 \mathrm{~mW}$ at the first failure point at an optimal $0.1 \%$ error rate, leading to a further $65.1 \%(6.06 \mathrm{~mW}$ vs. $17.35 \mathrm{~mW})$ power saving. With parallel processing (PP) feature enabled, the operating frequency can be scaled to $1 / 3$ of the original one. As a result, the voltage would be tuned to a much lower level. Finally, an additional $46.7 \%(3.23 \mathrm{~mW}$ vs. $6.06 \mathrm{~mW})$ power reduction is achieved. For 8-bits operands, the MP, DVS, and PP schemes can help save $87.4 \%(4.90 \mathrm{~mW}$ vs. $39.04 \mathrm{~mW}), 70.2 \%(1.46 \mathrm{~mW}$ vs. $4.90 \mathrm{~mW})$, and $55.5 \%(0.65$ vs. $1.46 \mathrm{~mW}$ ) power respectively.

Fig.2.16 shows the experimental results comparing the power savings associated to multi-precision, razor-based DVS and parallel processing schemes. We can see that multi-precision architecture achieves more aggressive supply voltage 


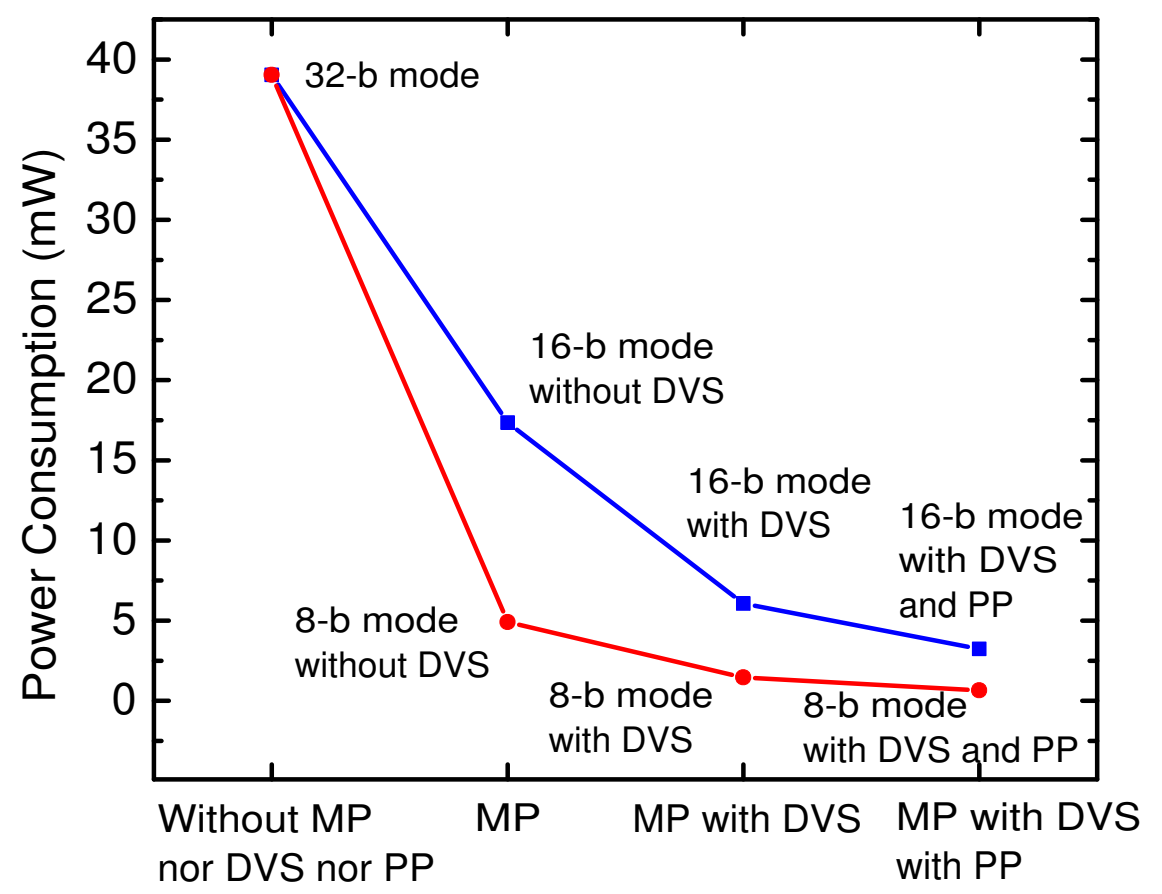

Figure 2.15: Experimental results of power consumption of different operating schemes. (MP: Multi-precision; DVS: Dynamic voltage scaling; PP: Parallelprocessing)

scaling than the fixed width architecture for improved power efficiency. As shown in Fig.2.16, power is reduced from $39.04 \mathrm{~mW} \rightarrow 6.06 \mathrm{~mW}$ in the 16-bit mode, from $39.04 \rightarrow 1.46 \mathrm{~mW}$ for the 8-bit mode. In contrast, power savings are less significant for the 32 -bit precision with a reduction from $39.04 \mathrm{~mW}$ down to $\rightarrow 21.52 \mathrm{~mW}$ ). In addition, the razor flip-flops help to reduce the operating voltage to the minimum possible level, resulting in a further power reduction of $26.1 \%(29.12 \mathrm{~mW}$ $\rightarrow 21.52 \mathrm{~mW}), 24.9 \%(8.07 \mathrm{~mW} \rightarrow 6.06 \mathrm{~mW})$ and $26.3 \%(1.98 \mathrm{~mW} \rightarrow 1.46 \mathrm{~mW})$ for 32-bit, 16-bit and 8-bit precision respectively. With parallel processing, the power reduction space is further enlarged.

Table 2.3 compares the performance of the fabricated prototype with related works: [17, 26, 27] are fixed precision fixed voltage schemes, [15, 28, 29] are multi-precision, fixed voltage schemes, and $[10,11]$ are multi-precision, multivoltage schemes. To compare the silicon area associated to each scheme, we chose to use the number of transistors because it constitutes a fair metric to compare different CMOS technology nodes. From the table, one can note that the proposed multiplier provides the most reconfigurability while exhibiting the smallest relative area. Compared to the designs with the same maximum word- 


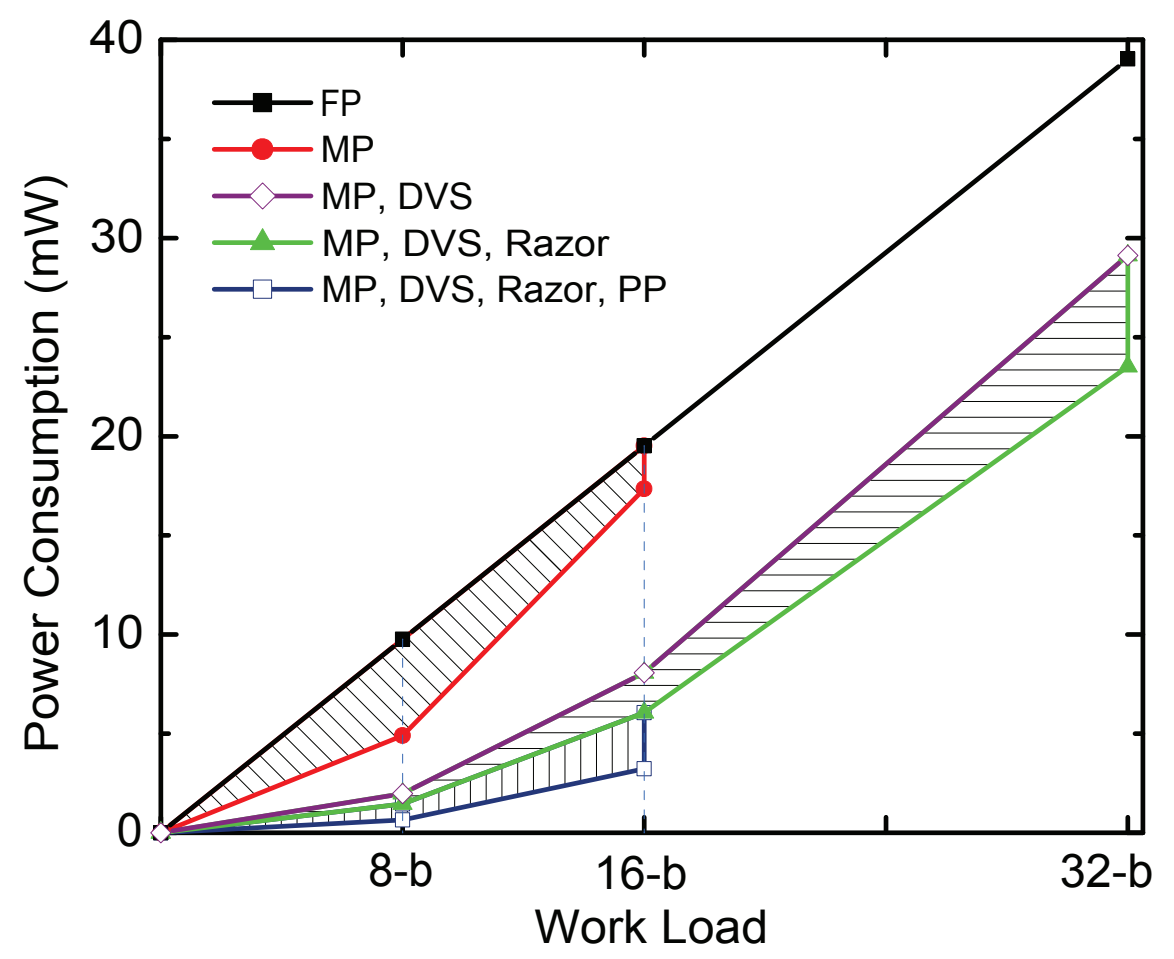

Figure 2.16: Experimental data of power savings associated to multi-precision, razor-based DVS and parallel processing schemes. (MP: Multi-precision; DVS: Dynamic voltage scaling; PP: Parallel-processing)

length of 32-bit [27, 29], our design occupies a much smaller area. For design [17] and design [15], their maximum word-length is 16-bit instead of 32-bit. If we assume that a $32 \times 32$-bit multiplier is built by $316 \times 16$-bit multipliers, then the area of the 32-bit multiplier is at least 3 times of that of the 16-bit multiplier, if we discard glue and reconfigurability logic. This analysis, shows that our design outperforms every other work in terms of silicon area and flexibility.

Table 2.3 also reports power results normalized (using the equation $P=$ $\left.C V^{2} f \alpha_{0-1}\right)$ when different technologies are used. Our multiplier also outperforms every other work in terms of power dissipation. In prior works, reconfigurability is always associated with increased silicon area and power consumption. In this chapter, we proposed an implementation, which not only provides multi-precision reconfigurable datapath, but also obtains a reduction in both silicon area and power compared to fixed-precision multipliers. 
TABLE III

Performance Comparison of proposed multiplier with related works

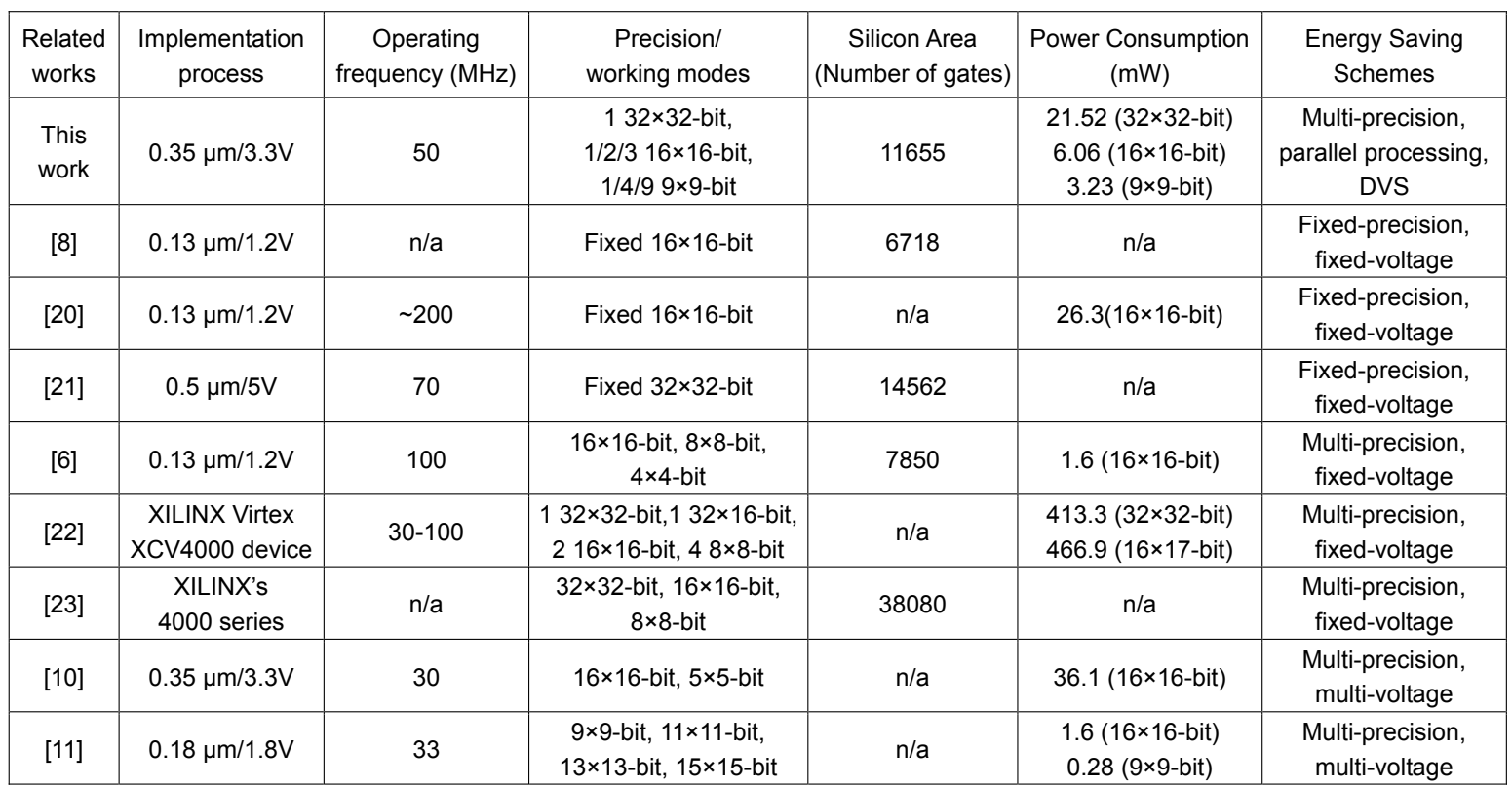

\subsection{Summary}

In this chapter, we proposed a multi-precision multiplier, which combines variable precision processing, scaled supply voltage and clock frequency, and parallel processing to efficiently reduce circuit's power consumption. We demonstrate that variable adaptive operation precision can allow aggressive scaling of supply voltage and clock frequency for improved power efficiency with minimum overhead. Experimental results show that $55.6-87.4 \%$ power reduction can be easily achieved by multi-precision approach. With razor-based DVS and DFS techniques, a further $65.1-70.2 \%$ power reduction could be obtained by operating the multiplier under minimum possible supply voltage and operating frequency. Parallel processing can bring extra 46.7-55.5\% reduction. The proposed multi-precision and dynamic voltage scaling multiplier provides a solution towards achieving computational flexibility and low power consumption for various general purpose low-power applications. 


\section{Chapter 3}

\section{Dedicated Operands Scheduler for Optimal Operation Voltages and Frequencies}

\subsection{Introduction}

In the previous section, we reported experimental results obtained using different data sets, each composed of randomly generated single-precision operands. However, in some applications like artificial neural network applications, the input data stream could include mixed-precision operands [8]. Although our multiplier provides three different precision modes (32×32-bit, $16 \times 16$-bit, $8 \times 8$-bit), the supply voltage would still have to transit dynamically between the minimum required voltage levels $V_{\min 32}, V_{\min 16}$, or $V_{\min 8}$ required for 32-bit, 16-bit and 8-bit operands respectively. From Fig.2.14, we can see that given a certain operating frequency, the difference among $V_{\min 32}, V_{\min 16}$, and $V_{\min 8}$ can be in the range of $0.1-0.65 \mathrm{~V}$. If the input data stream requires frequent supply voltage transitions, significant dynamic power would be dissipated, thereby undermining the benefits of dynamic voltage scaling. Furthermore, these transitions may not always be possible within one clock cycle. To minimize the overall power consumption, one needs to reduce the number of supply voltage transitions while still processing operands 
at the minimum required voltage level. To address this problem, we propose an input operands scheduler that will perform the following tasks: (i) re-order the input data stream such that same-precision operands are grouped together into a buffer (Fig.3.1); and (ii) find the minimum supply voltages $\left(V_{\min 32}, V_{\min 16}, V_{\min 8}\right)$ and operating frequencies $\left(f_{32}, f_{16}, f_{8}\right)$ for the 3 different-precision data groups to minimize the overall power consumption while still meeting the specified throughput.

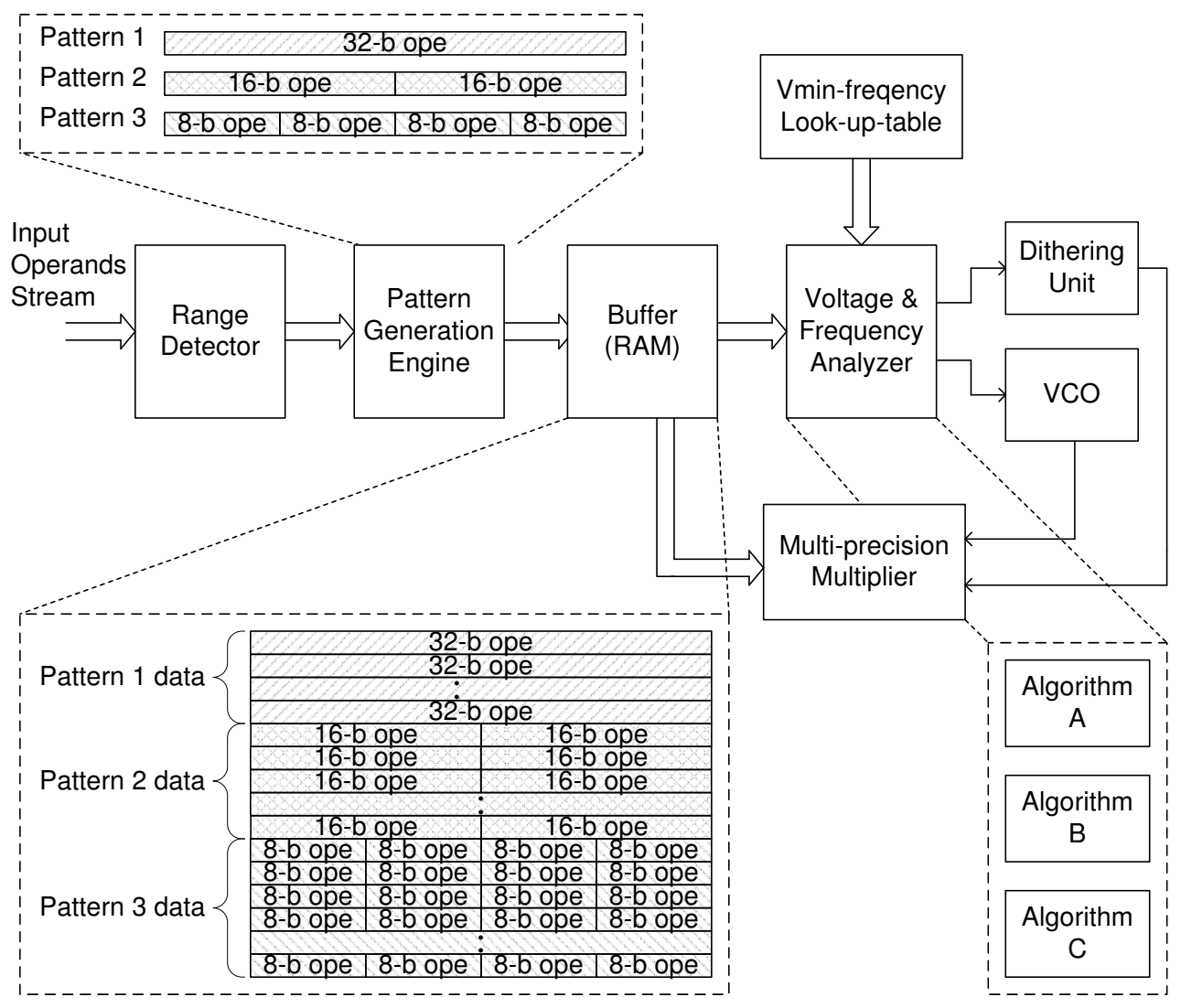

Figure 3.1: Block diagram of the input operands scheduler.

The block diagram of the input operands scheduler is shown in Fig.3.1. It is composed of an operand range detector, a pattern generation engine, a 2kbit buffer (RAM), and a frequency/voltage analyzer. The scheduler operates as follows. The inputs operands are first sent to the range detector, which classifies them according to their precision: 32-bit, 16-bit, or 8-bit. The classified data is then grouped by the pattern generation engine, which packs same-precision data into three different 32-bit data patterns (Fig.3.1): (i) pattern1 corresponds to original 32-bit input operand data, (ii) pattern2 combines two 16-bit operands 
data (with their redundant 16 MSBs removed), and (iii) pattern3 combines four 8-bit operand data (with their redundant 24 MSBs removed). At each clock cycle, a 32-bit data pattern can be processed, owning to the parallel processing capability of the proposed multiplier. This resembles the SIMD structure, and helps to put the multi-precision and parallel processing capability into real effect. As depicted in Fig.3.1, the 3 different data patterns are counted $\left(N_{32}, N_{16}\right.$ and $N_{8}$ ) and stored into a buffer, together with the respective voltages and clock frequencies at which they should be processed. For each full buffer, there will only be 2 transitions needed: $\left(V_{\min 8}, f_{8}\right)$ to $\left(V_{\min 16}, f_{16}\right)$, and $\left(V_{\min 16}, f_{16}\right)$ to $\left(V_{\min 32}, f_{32}\right)$. To limit the silicon area overhead, we chose a $2 \mathrm{k}$ RAM, which can store sixty 32-bit data patterns. The voltage/frequency analyzer specifies the values of $V_{\min 32}, V_{\min 16}, V_{\min 8}, f_{32}, f_{16}$ and $f_{8}$ to the dithering unit and VCO. The $V_{\text {min }}-f$ pairs were determined during the characterization of the chip and stored in the look-up table (Fig.3.1).

Given a random mixed-precision (32, 16 or 8-bit) input data stream and specified throughput $T$, our goal is to determine the voltages $\left(V_{\min 32}, V_{\min 16}, V_{\min 8}\right)$ and frequencies $\left(f_{32}, f_{16}\right.$ and $\left.f_{8}\right)$ at which each precision data group should be processed such that the total power consumption is minimized. In the following analysis, we consider the following four components of the total power consumption: the resistive power loss $P_{\text {dith_resistic_loss }}$ of the dithering unit, the switching power loss $P_{\text {dith_switching loss }}$ of the dithering unit, the dynamic power consumption $P_{\text {computation }}$ associated to the multiplication computation, and finally

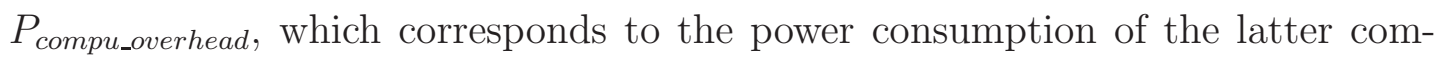
putation when carried out at voltage levels higher than the nominal Vmin. The expressions of the aforementioned four components of the total power consumption are given below:

$$
P_{\text {dith_resistic_loss }}=I_{\text {char }}^{2} R_{\text {on }}
$$

where $I_{\text {char }}$ is the charge current of the dithering unit, $R_{o n}$ is the equivalent resistance of the dithering switch. 


$$
P_{\text {dith_switching } \_ \text {oss }}=\frac{C_{g} V_{d d}^{2} f}{N}
$$

where $C_{g}$ is the gate capacitance of the dithering switch, $V_{d d}$ is the $3.3 \mathrm{~V}$ standard voltage, $N$ is the number of input data patterns.

$$
P_{\text {computation }}=C_{m} V_{\text {min }}^{2} f
$$

where $C_{m}$ is the effective capacitance of the multiplier, $V_{\min }$ is the applied minimum supply voltage $\left.V_{\min 8}\right)$, and $f$ is the applied operating frequency.

$$
P_{\text {compu_overhead }}=\frac{\int P d t}{T}=\frac{\int C_{m} V^{2} f d V}{T}
$$

where $V$ is the dithering unit output, which fluctuates around $V_{\min }$. $T$ is the charge time period, which is inversely proportional to the operating frequency.

The overall power consumption is thus given by:

$$
\begin{gathered}
P_{\text {overall }}=P_{\text {computation }}+P_{\text {compu_overhead }} \\
\quad+P_{\text {dith_resistic_loss }}+P_{\text {dith_switching } \_ \text {oss }}
\end{gathered}
$$

In the following, we present three different algorithms to reduce the overall power consumption. Each of these algorithms constitutes a different approach to process the mixed-precision data held in the operands buffer. The performance of each algorithm will be evaluated using a mixed-precision data set of 120000 randomly operands, with a third of the total data for each precision $(8,16$ and 32-bit). In the following, the specified throughput $T$ for the proposed $32 \times 32$ bit multiplier is $64 F$ (Mbits/sec), where $F$ refers to the multiplier's operating frequency. 


\subsection{Algorithms and Performance Comparison}

\subsubsection{Algorithm A for Low Cost Implementation}

In the first algorithm, the multiplier throughput $T=64 F$ is kept constant by fixing the operating frequencies $\left(f_{32}, f_{16}\right.$ or $\left.f_{8}\right)$ of each precision-data group (32, 16 or 8-bit) to:

$$
f_{32}=F, f_{16}=\frac{F}{2}, f_{8}=\frac{F}{4}
$$

where $F$ is the multiplier's operating frequency. This is because the throughput in $8 \times 8$-bit multiplication mode is four times that of the $32 \times 32$-bit multiplication mode and double that of the $16 \times 16$-bit multiplication mode, as a result of the multiplier parallel processing. The minimum supply voltage $\left(V_{\min 32}, V_{\min 16}\right.$ or $\left.V_{\min 8}\right)$ associated to each operating frequency $\left(f_{32}, f_{16}\right.$ or $\left.f_{8}\right)$ is determined through a $V_{\min }-f$ look-up-table.

Algorithm A shows its limitations when 32-bit operands are processed first. As depicted in Fig.3.2, once all $N_{32}$ operands have been processed, the supply voltage $\left(V_{n}\right)$ needs to decrease rapidly from point $\mathrm{A}\left(V_{\min 32}\right)$ to point $\mathrm{B}\left(V_{\min 16}\right)$ at which all $N_{16}$ 16-bit operands should be processed. If $N_{16}$ is too small, most 16-bit operands will be actually processed in section $\mathrm{A}-\mathrm{B}$, that is at a voltage possibly much higher than the minimal $V_{\min 16}$ level. Similarly 8-bit operands could be processed in section $\mathrm{C}-\mathrm{D}, \mathrm{B}-\mathrm{C}$, or even $\mathrm{A}-\mathrm{B}$ for the worst case. The above contributes to increasing $P_{\text {compu_overhead }}$. The overall power performance of algorithm A is summarized in Table.3.1. Compared to the non-scheduled but voltage scaled $32 \times 32$-bit situation $(32 \times 32$-bit mode must be chosen because there are one third of 32 -bit operands), a $74 \%$ power reduction is achieved. 


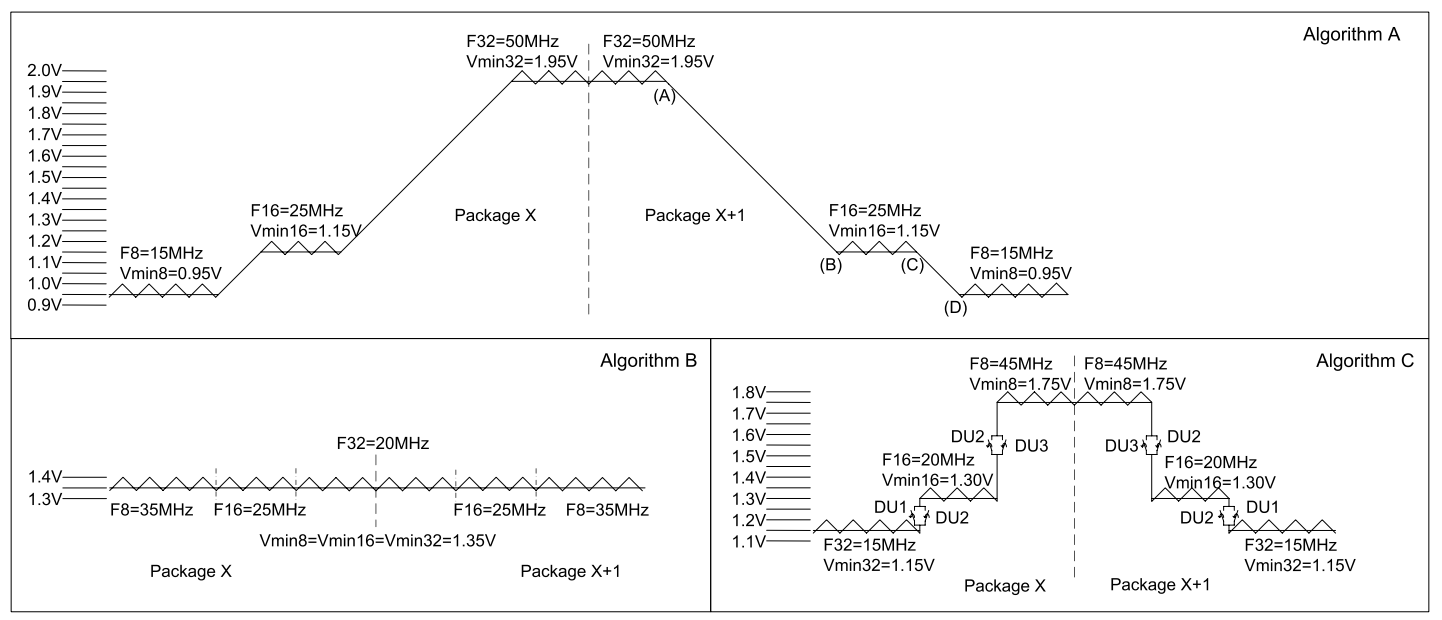

Figure 3.2: Principles of algorithm A, B and C.

TABLE 3.1

Detailed Power Performance of Different Scheduling Algorithms

\begin{tabular}{|c|c|c|c|c|c|}
\hline Algorithm & P_computation & P_compu_overhead & P_dith_resistic_loss & P_dith_switching_loss & P_overall \\
\hline A & $3.034 \mathrm{~mW}$ & $3.159 \mathrm{~mW}$ & $0.059 \mathrm{~mW}$ & $1.715 \mathrm{~mW}$ & $8.255 \mathrm{~mW}$ \\
\hline B & $2.266 \mathrm{~mW}$ & $2.565 \mathrm{~mW}$ & $0.839 \mathrm{~mW}$ & $1.663 \mathrm{~mW}$ & $6.578 \mathrm{~mW}$ \\
\hline C & $1.682 \mathrm{~mW}$ & $1.843 \mathrm{~mW}$ & $0.062 \mathrm{~mW}$ & $0.975 \mathrm{~mW}$ & $4.561 \mathrm{~mW}$ \\
\hline
\end{tabular}

\subsubsection{Algorithm B for Zero Voltage Transition}

This algorithm removes all transitions of the power supply voltage by making $V_{\min 32}, V_{\min 16}$, and $V_{\min 8}$ equal and adjusting $f_{32}, f_{16}$ and $f_{8}$ such that the overall throughput is kept unchanged, that is we need to have:

$$
\frac{64 N_{32}+128 N_{16}+256 N_{8}}{\frac{N_{32}}{f_{32}}+\frac{N_{16}}{f_{16}}+\frac{N_{8}}{f_{8}}}=64 F
$$

From a look-up-table, we can get the $V_{\min }-f$ relationship:

$$
\begin{gathered}
V_{\min 32}=\psi_{32}\left(f_{32}\right) \\
V_{\min 16}=\psi_{16}\left(f_{16}\right) \\
V_{\min 8}=\psi_{8}\left(f_{8}\right)
\end{gathered}
$$


Since Algorithm B keeps the supply voltage constant:

$$
\psi_{32}\left(f_{32}\right)=\psi_{16}\left(f_{16}\right)=\psi_{8}\left(f_{8}\right)=V
$$

the operating frequencies $f_{32}, f_{16}$ and $f_{8}$ can be determined by using equations (3.7) and (3.11). For example, when $F$ is set to $50 \mathrm{MHz}$, the values for $V$, $f_{32}, f_{16}$ and $f_{8}$ were found to be $1.35 \mathrm{~V}, 20 \mathrm{MHz}, 25 \mathrm{MHz}$, and $35 \mathrm{MHz}$ respectively. The overall power performance of algorithm B is given in Table.3.1. Due to the complete removal of voltage transitions, the $P_{\text {compu_overhead }}$ is reduced. At the same time, because of holistic planning, the dynamic computation power is also optimized to a lower level.

\subsubsection{Algorithm C for Minimum Power}

Although algorithm B removes power supply voltage transitions by setting a single voltage level $V$, there may well be more power saving combinations of power supply voltages and operating frequencies. $\left(V_{\min 32}, f_{32}\right),\left(V_{\min 16}, f_{16}\right)$ and $\left(V_{\min 8}, f_{8}\right)$. The aim of algorithm $\mathrm{C}$ is to find such an optimum for reduced power consumption. For sake of simplicity, we will here only seek to minimize the dynamic power dissipated associated to computations:

$$
\begin{array}{r}
P=C V^{2} f \\
=C_{m 32} V_{m i n 32}^{2} f_{32}+C_{m 16} V_{m i n 16}^{2} f_{16}+C_{m 8} V_{m i n 8}^{2} f_{8} \\
=\chi\left(f_{32}, f_{16}\right)
\end{array}
$$

Given that the $V_{m i n}-f$ relationships are known (Equations 3.8-3.10), one could find the minimum of the above expression for the specified throughput (Equation 3.7). For example, when $F$ is set to $50 \mathrm{MHz}$, the values for $\left(V_{\min 32}\right.$, $\left.f_{32}\right),\left(V_{\min 16}, f_{16}\right),\left(V_{\min 8}, f_{8}\right)$ were found to be $(1.15 \mathrm{~V}, 15 \mathrm{MHz}),(1.30 \mathrm{~V}, 20 \mathrm{MHz})$, 
and $(1.75 \mathrm{~V}, 45 \mathrm{MHz})$ respectively. The overall power performance of algorithm $\mathrm{C}$ is given in Table.3.1. To remove transitions among the different $V_{\text {mins }}$, one could use 3 discrete dithering units to generate $V_{\min 32}, V_{\min 16}$, and $V_{\min 8}$. As seen in Table.IV, this reduces both $P_{\text {computation }}$ and $P_{\text {compu_overhead }}$ significantly, with an overall power reduction of $86 \%$, compared to the standard voltage scaled $32 \times 32$-bit multiplier. Table.3.1 shows that algorithm $\mathrm{C}$ exhibits the least power consumption. However, it requires two additional dithering units to help realize the discrete power supply. Algorithm B provides a better trade-off between area and power.

\subsubsection{Scheduling Overhead Evaluation}

The implementation overhead for the operands scheduler comes from: the range detector and pattern generator, the RAM buffer, and the voltage and frequency analyzer. The range detector is realized by a number of XOR gates to detect the successive 0 's or 1's from the beginning of the operands. The pattern generator uses buffer with the same size as the buffer used for pattern storage to sort the data after range detection. The voltage and frequency analyzer is mainly based on the Vmin-frequency Look-up-table. Algorithm A is the simplest and can be realized by just looking up the LUT. Algorithm C is the most complex one, however because the available solutions are discrete values, there is no real need to solve the equations. To simplify the computation, some approximation methods can be applied with no effect on the final results. For example, in the first step of solving Eq.(3.12), we could round the $N_{32}, N_{16}, N_{8}$ values to their nearest multiple of 5 , then only a limited number (up to 5) of possible voltage and frequency combinations are obtained. The optimal pair can be found by comparing their power consumption using Eq. (3.16).

The largest overhead comes from the RAM blocks, which is used for data sorting and data storage. Based on the area and power features of single port RAM in AMIS $0.35 \mu m$ technology, the overall area overhead of all buffers is about $0.23 \mathrm{~mm}^{2}$, while the overall power overhead is about $0.55 \mathrm{~mW}$. 


\subsubsection{Potential Applications}

A potential application for our fine-granularity scheduling and execution multiplier system is multimedia sensor network applications. A number of tasks for example, image processing (eg. data from camera), audio signals processing (eg. data from microphone), communication tasks (eg. data from antenna), and CPU programme, etc, need to be executed during same period of time. Such tasks require low-power, low-cost processing, but cannot tolerate high latencies.

For the host CPU, our multiplier system is only an Accelerated Processing Unit (ASU). It is not possible to completely rely on the compiler to do the static optimization of task scheduling. The dynamic optimization at the hardware level plays an important role in the whole system. For example, the camera outputs 8-bit or 16-bit data, the antenna outputs 16-bit data, the microphone outputs up to 24-bit data, and the CPU programme (eg. encryption) outputs 32-bit, 64-bit, even 128-bit data. All of these data from different sensor nodes may need to be processed at the same slot of time using a single ASU. Under these circumstances, the multi-precision and parallel processing ASU becomes an attractive solution for low-cost low-power and high performance processing. The data of different sensor nodes can be fed into the multiplier at the same time. Based on these multi-stream multi-precision data, the window-by-window operands scheduling can be performed to achieve the minimum possible power consumption and prescheduled low latency requirements.

\subsection{Summary}

In this chapter, we proposed an operands scheduler unit to analyze and rearrange the input data to help the multiplier to automatically configure itself to work in different precision modes. Three different scheduling algorithms are proposed. With different gains and overheads. Algorithm $\mathrm{C}$ has least power consumption, however, it needs 2 more dithering units. If silicon area is not critical, algorithm $\mathrm{C}$ can yield best power performance. If area is an important consideration, algorith- 
m B can give a better trade-off between area and power. When augmented with the dedicated operands scheduler, an additional $46.2 \%$ power saving is achieved. The proposed multi-precision and dynamic voltage scaling multiplier provides a solution towards achieving computational flexibility and low power consumption for various general purpose low-power applications. 


\section{Chapter 4}

\section{A Compact Multi-Reset Integration Digital Pixel Sensor}

\subsection{Introduction}

Complementary metal-oxide-semiconductor (CMOS) image sensors are now part of our everyday's life covering a wide spectrum of applications from cell-phone cameras, webcams, digital cameras, video games to security and automotive ap-

plications [30]. The successful deployment of CMOS image sensors over the charge coupled devices (CCD) technology is mainly due to reduced cost and power consumption, as well as higher integration and on-chip processing capabilities, which are critical for mobile applications.

Among CMOS image sensors, two mainstream architectures can be distinguished depending on where the analog-to-digital (A/D) conversion is achieved. Active pixel sensors (APS) perform the A/D conversion outside the pixel array using a per-array or per-column readout scheme. On the other hand, digital pixel sensors (DPS) integrate the A/D conversion at the pixel level. DPS, while giving flexibility of use, enable massive parallel A/D conversion at the pixel level. In addition, since A/D conversion is performed at the sensor level, DPS can offer improved noise figure [31]. Previous DPS implementations were based on either pulse frequency modulation (PFM) [32] or pulse width modulation (PWM) [33] 
schemes to digitize the voltage of the photodiode. The PWM scheme uses a comparator output signal to write in a global counter value in the pixel-level memory, while the PFM scheme uses the comparator output to enable an increment of the pixel-level counter. Both PFM and PWM schemes are typically power hungry, due to the high switching activity at the pixel level. In addition, a major drawback of both schemes is reduced fill-factor because of the pixel-level memory. The area and power overhead due to the pixel-level memory are substantial and can account for up to $50 \%$ of the total silicon area overhead. Several attempts were made to reduce the memory needs at the pixel level. They include the use of an address event representation (AER) scheme [34], which completely removes the memory from the pixel at the cost of increased complexity and the introduction of timing errors due to collisions occurring when several pixels attempt to access the output data bus simultaneously. Another reported solution consists of compressing the data even before storage as proposed in [35]. This last solution drastically reduces the pixel area but at the cost of reduced signal-to-noise ratio (SNR) due to the use of lossy compression scheme. In [36], the output of the comparator is sampled and stored using a single bit register cell per pixel. All register cells are connected in series to form a scan chain, with the whole array scanned after each sampling operation. While only one bit pixel-level memory is required, this architecture increases power consumption as the number of samples, which is a strong function of the resolution.

In digital pixel sensors (DPS), memory elements typically occupy large silicon area of the pixel, which significantly reduces the pixel's fill factor while increases its size, power and cost. In this chapter, we propose to reduce DP$\mathrm{S}$ memory's area and power overhead and reduce memory requirements with a multi-reset integration scheme, that employs a dynamic memory instead of the traditional large 6T-SRAM cell. The operation of the proposed DPS takes advantage from the chronological change of the code, which results in reduced memory needs without affecting the sensor's sensitivity. In the proposed implementation, a 4-bit in-pixel memory is used to reduce the pixel size, and an 8-bit resolution is achieved with this multi-reset scheme. In addition, full complementary metal-oxide-semiconductor (CMOS) 2T DRAM and selective refresh scheme are 
adopted to implement the memory elements and further increase area savings. This chapter presents the proposed multi-reset integration methodology and its implementation with dedicated memory circuits. The proposed architecture is validated by a prototype chip fabricated using AMS $0.35 \mu \mathrm{m}$ CMOS technology. Reported experimental results are compared with prior works.

This chapter is organized as follows: Section 2 introduces the PWM time domain DPS and its conversion time analysis. Section 3 is devoted to the multireset integration methodology and a discussion about the trade-offs involved in terms of speed, area and memory size. Section 4 describes the multi-reset integration DPS architecture, the pixel circuitry and the 2T DRAM implementation and sensing scheme. Section 5 provides an analysis of power consumption as well as power reduction techniques. Section 6 presents the prototype implementation and experimental measurements. Section 7 concludes this work.

\subsection{Time-Domain PWM DPS}

\subsubsection{Conventional Architecture}

The conventional pixel architecture of a DPS using the PWM technique is shown in Figure 4.1. The pixel comprises a photodiode $P_{d}$, a reset transistor $A R$, a voltage comparator, a memory unit and a feedback circuit to perform the autoreset of the photodiode. Outside the pixel array, a timing control unit and a global counter are required to perform the conversion of the light intensity into a digital code. A read-out circuit is also needed to read the contents from the memory and to output the data to the processing unit.

The integration phase starts by disabling the precharge to the supply voltage of the photodiode i.e. when the reset signal transits from 0 to $V_{d d}$. Meanwhile, a global counter located outside the pixel array is simultaneously enabled. The voltage $V_{d}$ of the photodiode node loaded with a capacitance $C_{p d}$ starts decreasing proportionally to light intensity due to the photo-generated current $I_{d}$ through the photodiode $P_{d}$. When the voltage $V_{d}$ reaches a reference voltage $V_{r e f}$, the 


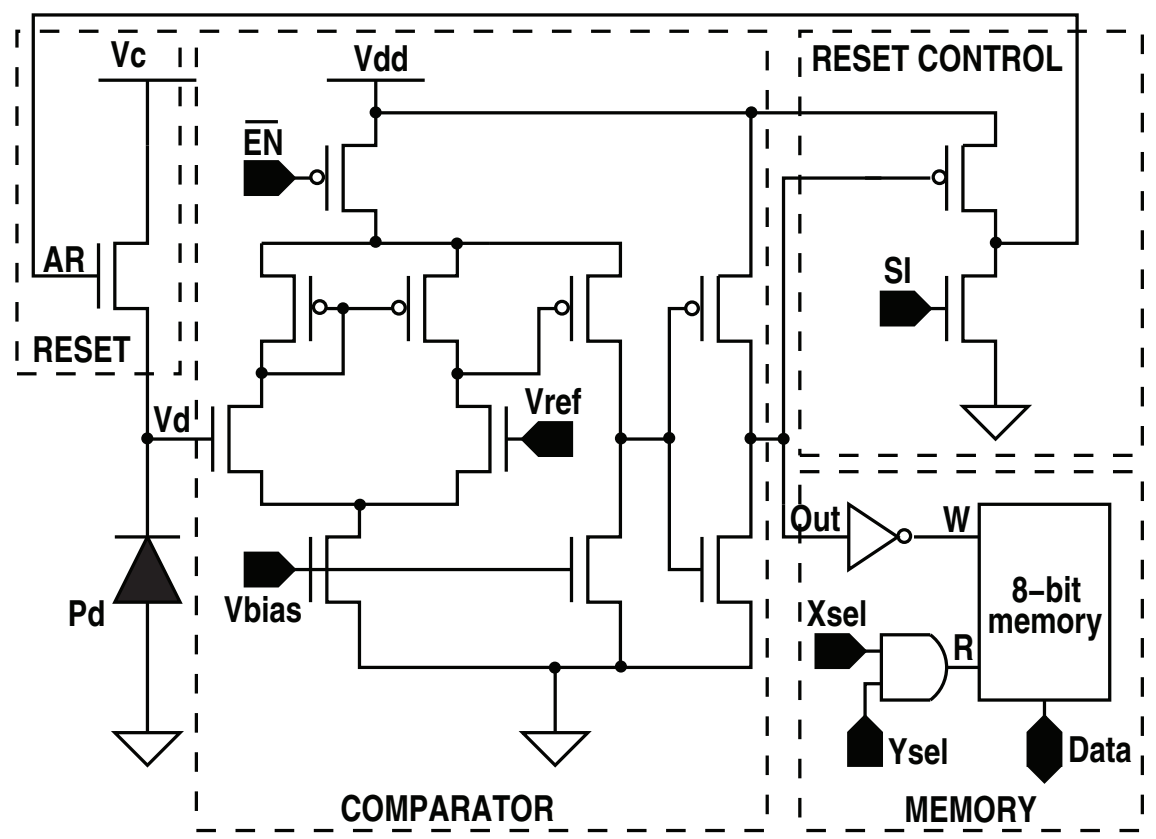

Figure 4.1: Schematic view of the conventional pixel [37].

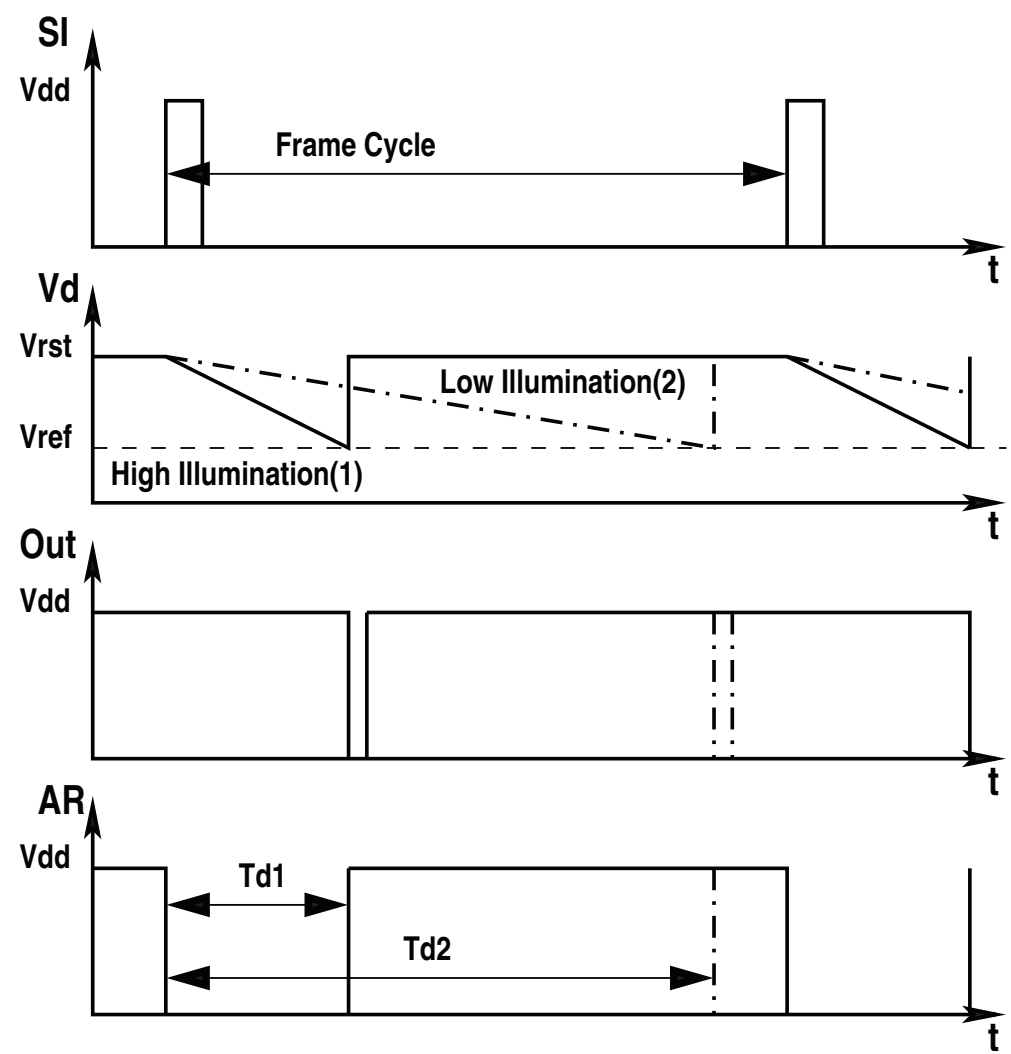

Figure 4.2: The timing diagrams of the conventional pixel. 
output of the comparator switches high and the counter value is written to the pixel-level memory. The reset of the photodiode is performed automatically after the photodiode voltage has reached the reference level $V_{r e f}$. The code written in the memory is a digitized value of the time required for $V_{d}$ to reach $V_{r e f}$, which is a function of the light intensity. Using first order approximation, the integration time is expressed as:

$$
T_{i n t}=\frac{C_{p d}\left(V_{d d}-V_{r e f}\right)}{I_{d}}=\frac{\alpha}{I_{d}}
$$

From Eq. (4.1), one must notice that the integration time is inversely proportional to the photo-generated current $I_{d}$. To perform the quantization of the integration time, the global counter is used to provide the quantization boundaries for the time to digital conversion. The non-linearity between the photocurrent and the integration time can be compensated by adapting the frequency of the global counter as shown in Figure 4.3 and Figure 4.4. On one hand, Figure 4.3 represents a uniform time domain quantization leading to a non-linear response from the sensor as the photocurrent boundary quantization steps are non-linear. On the other hand, the non-uniform time domain quantization depicted in Figure 4.4 enables to linearize the conversion of photocurrent into digital code as the photocurrent boundary quantization steps are now linear.

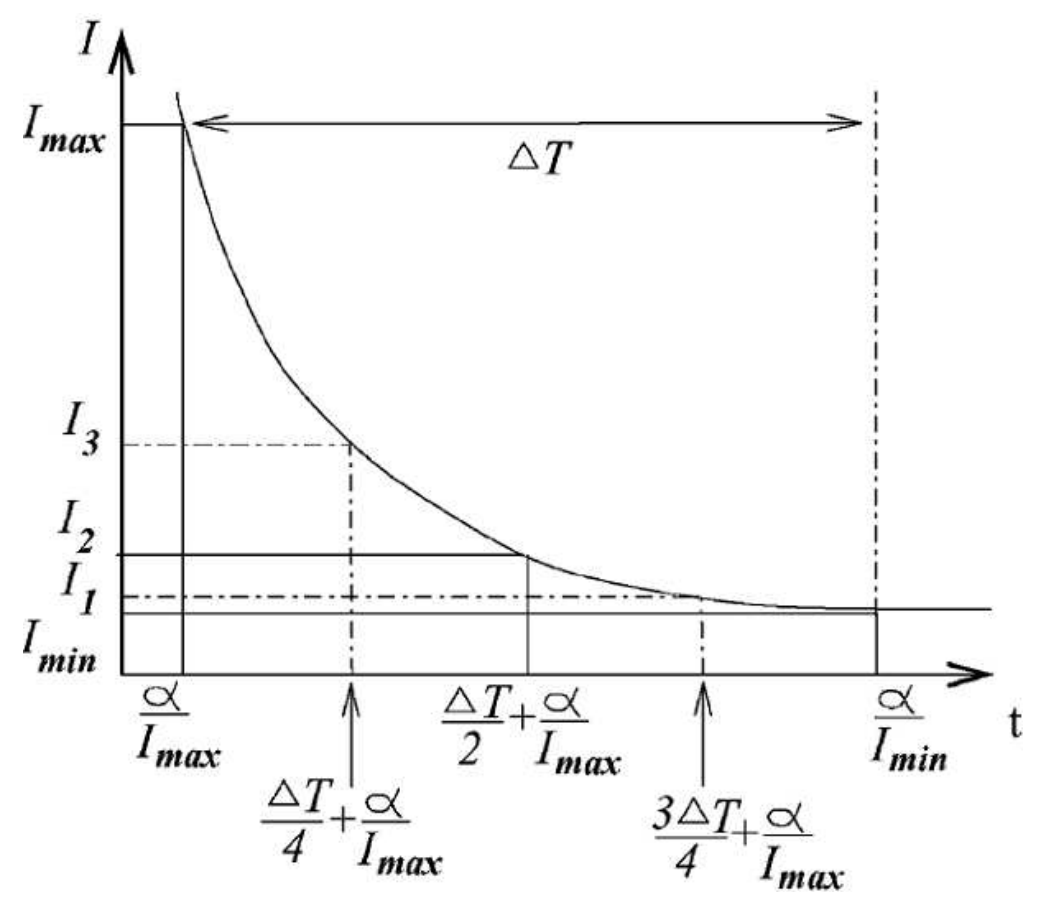

Figure 4.3: Uniform time domain quantization. 


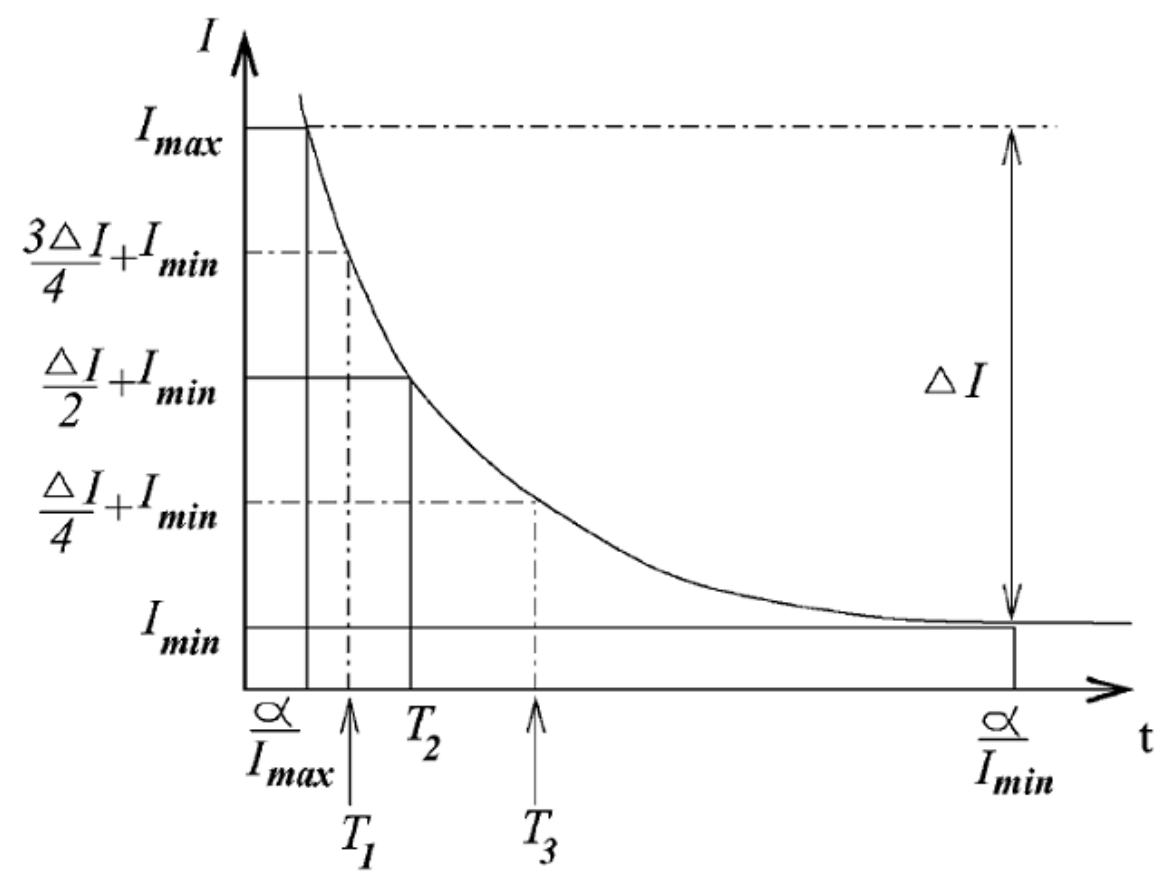

Figure 4.4: Non-uniform time domain quantization.

\subsubsection{Conversion Time Analysis}

A PWM coding scheme encodes the illumination level information of each pixel in a single pulse. This pulse width represents the time $T_{\text {int }}$ required to discharge a photodiode from $V_{d d}$ to $V_{\text {ref }}$. In order to convert the pulse into digital code, a time code generated by the global counter is written into the embedded memory once the pulse is detected. There are two quantization approaches to convert the PWM pulse signal into digital code. The first approach, referred to as uniform time domain quantization (UQ), which provides sampling times (or boundary quantization levels) from $T_{\min }$ to $T_{\max }$ uniformly. The second approach referred to as non-uniform time domain quantization (NUQ), resolves the sampling time in order to form a uniformly quantized photocurrents. A non-linear time-domain quantizer is therefore required in order to compensate for time-to-photocurrent non-linearity.

\subsubsection{Uniform Time-Domain Quantization}

Uniform time domain quantization scheme [37] divides the time within the boundaries $T_{\min }$ and $T_{\max }$ equally into $2^{n}$ quantization levels, where $n$ represents the 
number of bits or resolution. Therefore, the time width of each quantization level $\triangle T$ can be expressed as:

$$
\triangle T=\frac{T_{\max }-T_{\min }}{2^{n}}
$$

While the relationship between the quantization level $\xi_{U Q}(n, t)$ and the time width of the PWM pulse $t$ is illustrated in the following equation:

$$
\begin{aligned}
\xi_{U Q}(n, t) & =\frac{t-T_{\min }}{\triangle T} \\
& =\frac{2^{n}\left(t-T_{\min }\right)}{T_{\max }-T_{\min }}
\end{aligned}
$$

Using Eq. (4.3) the conversion between the quantization level $\xi_{U Q}\left(n, I_{d}\right)$ and the discharge photocurrent $I_{d}$ of the photodiode can be expressed as following:

$$
\begin{aligned}
\xi_{U Q}\left(n, I_{d}\right) & =\frac{2^{n}\left(\frac{\alpha}{I_{d}}-\frac{\alpha}{I_{d \max }}\right)}{\frac{\alpha}{I_{d \min }}-\frac{\alpha}{I_{d \max }}} \\
& =\frac{2^{n}\left(\frac{I_{d \max } I_{d \min }}{I_{d}}-I_{d \min }\right)}{I_{d \max }-I_{d \min }}
\end{aligned}
$$

Assuming that $I_{d \max } \gg I_{d m i n}$, Eq. (4.4) can be approximated as:

$$
\xi_{U Q}\left(n, I_{d}\right) \approx 2^{n} I_{d \min }\left(\frac{1}{I_{d}}-\frac{1}{I_{d \max }}\right)
$$

Eq. (4.5) suggests that, the quantization level is inversely proportional to the discharge current under the UQ scheme, as $\frac{I_{\text {dmin }}}{I_{d \max }}$ is a constant. Indeed, it also suggests that the UQ scheme is sensitive to the discharging current close to $I_{d m i n}$, shown in Figure 4.5. This suggests that the UQ scheme focuses on quantizing the low illumination levels, while the high illumination levels are not properly covered.

\subsubsection{Non-uniform Time-Domain Quantization}

Non-uniform time domain quantization scheme resolves the quantization levels in order to form linearly distributed photocurrents $\Delta I$ within the boundaries $I_{d m i n}$ 


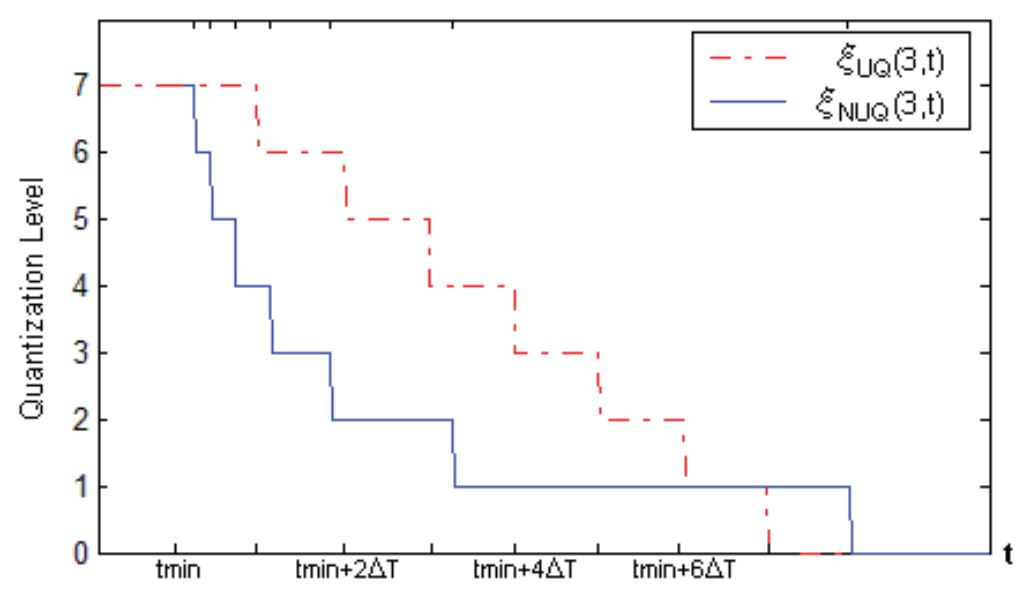

Figure 4.5: 3bits UQ and NUQ in terms of time.

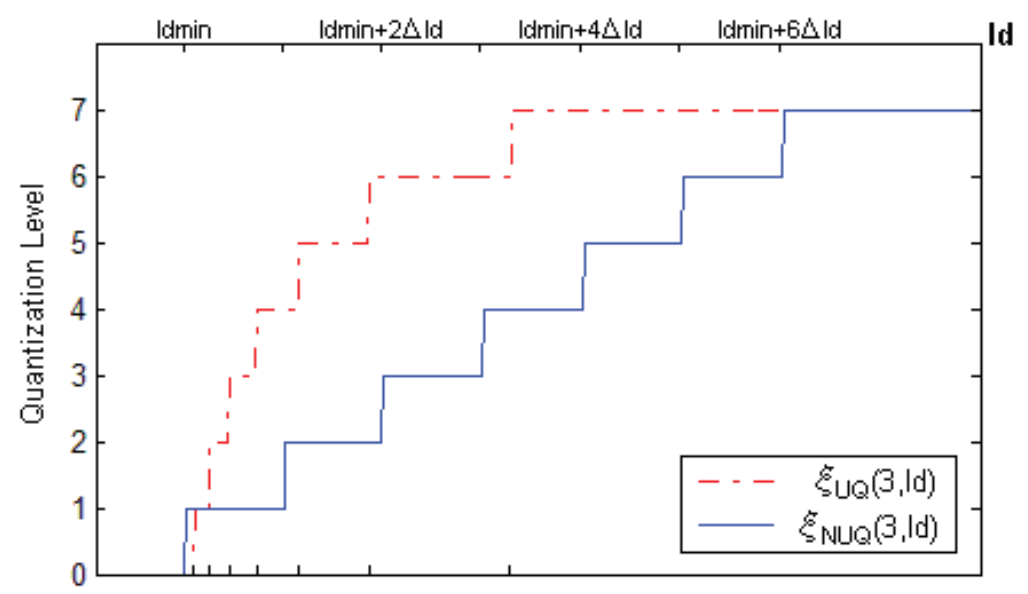

Figure 4.6: 3bits UQ and NUQ in terms of discharging current. 
and $I_{d \max }$.

$$
\triangle I=\frac{I_{d \max }-I_{d \min }}{2^{n}}
$$

The photocurrent $I_{d}$ can be converted to its dedicated quantization level $\xi_{N U Q}\left(n, I_{d}\right)$ as follows:

$$
\begin{aligned}
\xi_{N U Q}\left(n, I_{d}\right) & =\frac{I_{d}-I_{d \min }}{\triangle I} \\
& =\frac{2^{n}\left(I_{d}-I_{d \min }\right)}{I_{d \max }-I_{d \min }}
\end{aligned}
$$

From Eq. (4.7), we can note that the relationship between the time and the quantization level $\xi_{N U Q}(n, t)$ can be expressed as:

$$
\begin{aligned}
\xi_{N U Q}(n, t) & =\frac{2^{n}\left(\frac{\alpha}{t}-\frac{\alpha}{T_{\max }}\right)}{\frac{\alpha}{T_{\min }}-\frac{\alpha}{T_{\max }}} \\
& =\frac{2^{n}\left(\frac{T_{\max } T_{\min }}{t}-T_{\min }\right)}{T_{\max }-T_{\min }}
\end{aligned}
$$

In contrast to UQ scheme, Eq. (4.8) suggests that, the NUQ quantization levels are inversely proportional to the sampling time. Figure 4.6 shows that the NUQ provides an evenly distributed photocurrent sampling boundaries.

\subsection{The Proposed Multi-Reset Integration (MRI) Scheme}

In order to reduce the silicon area of the pixel and improve the fill factor, a multireset integration (MRI) scheme is proposed in this work to reduce the memory needs at the pixel level. This section presents the concept of the MRI and discusses the trade-offs in terms of delay overhead and size of the pixel memory. 


\subsubsection{MRI Concept}

The proposed integration scheme takes advantage of the sequential way the illumination level is digitized. The MRI scheme can be interpreted as performing the integration process several times in order to resolve each bit of the illumination code sequentially, from the most significant bit (MSB) to the least significant bit (LSB), as shown in Figure 4.7. During each new integration phase, only a sub-set of the $\mathrm{n}$ bits are stored in the pixel reducing the memory requirements. Between two integration periods, the content of the pixel memory is scanned out of the array, allowing for the remaining bits of the code to be stored during the successive integration phases. The required number of bits for the memory is therefore reduced by a factor proportional to the number of iterations. For example, considering a resolution of 8 bits and a single bit memory, 8 successive resets will be required. In order to reduce the delay overhead caused by the successive integration periods, it is possible to define timing boundaries for which the value of the concerned bit of the code is resolved allowing to optimize the corresponding integration duration for each bit.

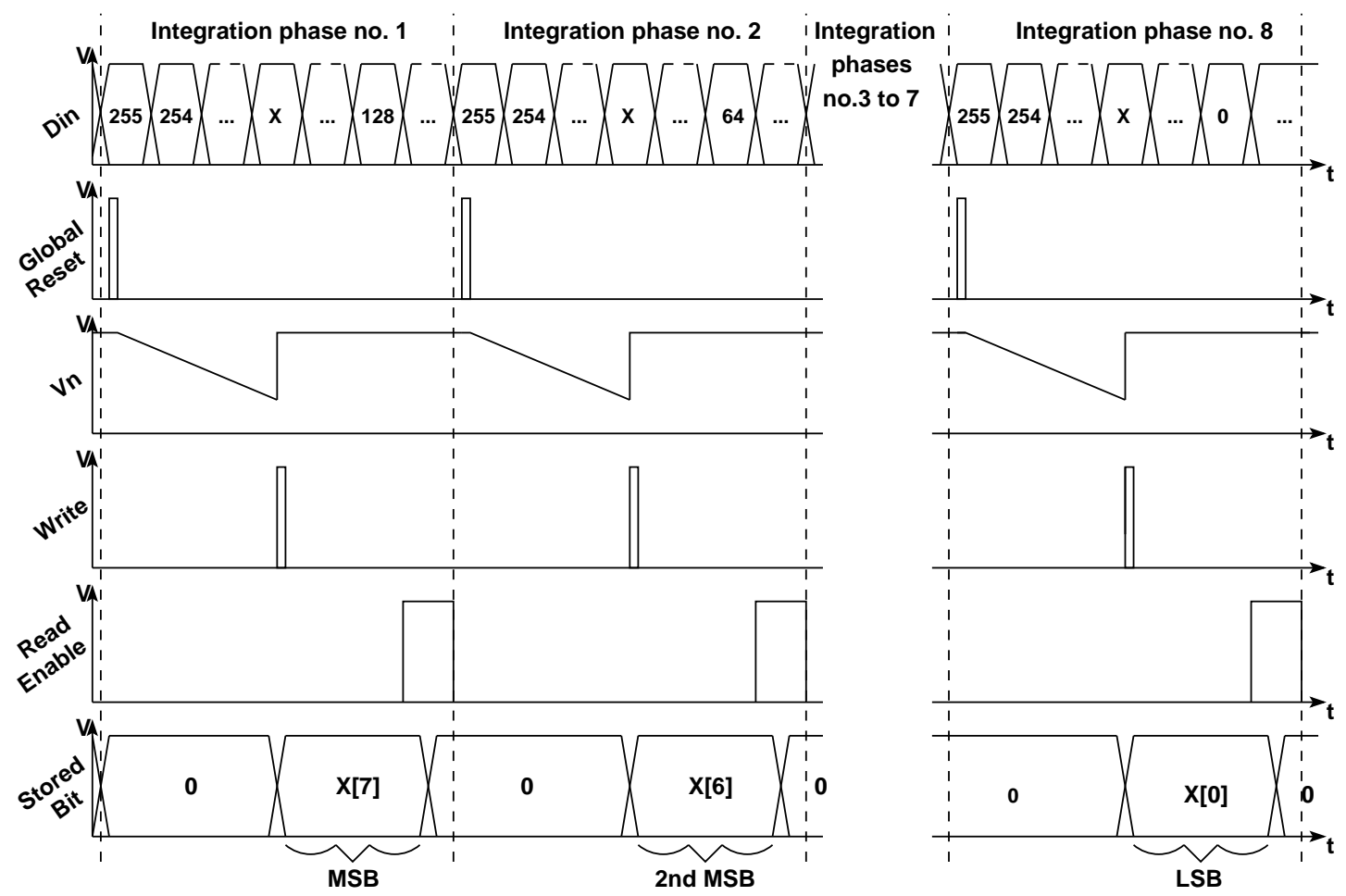

Figure 4.7: Timing diagram of the multi-reset integration scheme.

Assuming the photocurrent is quantized into $2^{n}$ values, with $n$ as the reso- 
lution. The quantization levels $N_{Q T}$ covered by each bit can be expressed as:

$$
N_{Q T}(i)=2^{n}-2^{i}+1 \quad i \in\{0, n-1\}
$$

where bit(0) represents the LSB and bit(n-1) represents the MSB. The quantization levels coverage of the MSB is only half of the total quantization levels. Therefore, the optimized integration time for the bits closer to the MSB is much shorter than those towards the LSB. Indeed, derived from Eq. (4.1), the optimized duration of the partial integration phase $T_{i n t}$ required to obtain the bit number $\mathrm{i}$ of the code is given by:

$$
\begin{aligned}
T_{\text {int }}(i) & =\frac{\alpha}{I_{\max }-\frac{2^{n}-2^{i}+1}{2^{n}}\left(I_{\max }-I_{\min }\right)} \\
& =\frac{\alpha}{I_{\min }}\left(\frac{1}{1+\left(2^{i-n}-2^{-n}\right)\left(\frac{I_{\max }}{I_{\min }}-1\right)}\right) \\
A s \frac{I_{\max }}{I_{\min }} \gg 1, & \\
& \approx T_{\max }\left(\frac{1}{1+\left(2^{i-n}-2^{-n}\right)\left(\frac{I_{\max }}{I_{\min }}\right)}\right)
\end{aligned}
$$

where $T_{\max }=\frac{\alpha}{I_{\min }}$ is the maximum integration time for $\mathrm{n}$ bits resolution. Under the $I_{\max } \gg I_{\min }$ condition, Eq. (4.10) illustrates that integration time decreases exponentially when i increases. Assuming 1-bit pixel memory, getting the $\mathrm{N}$ bits successively will give a total integration time $T_{\text {total }}$ that corresponds to the sum of the durations of each partial integration phase $T_{i n t}$. Therefore, the actual integration time of the proposed scheme is expressed by:

$$
T_{\text {total }}=T_{\max } \sum_{i=0}^{n-1} \frac{1}{1+\left(2^{i-n}-2^{-n}\right)\left(\frac{I_{\max }}{I_{\min }}\right)}
$$

From this equation, the total time required to realize the integration with the multi-reset integration scheme is much shorter than $n \times T_{\max }$. It can also be noted that the time required to scan the values out is not accounted for in this equation. However, this time can be reduced significantly using parallel and highspeed readout and remains negligible compared to the total integration time. In addition, the read-out phase can be interleaved with the integration phase. Figure 
4.8 takes 2-bit resolution as example and assumes $I_{\max } / I_{\min }=100$. Using Eq. (4.10), $T_{\text {int_MSB }}=\frac{1}{26} T_{\max }, T_{\text {int_LSB }}=T_{\max }$. It is possible to resolve the MSB at a $1 / 26$ fraction of the integration period followed by the second bit or the LSB after one integration period. Originally, under the MRI scheme, $2 T_{\max }$ are required to obtain the MSB and the LSB values. In this scheme, using the optimum timing boundaries, the total integration time is reduced by $48 \%$. These optimized timing boundaries are used to reset each partial integration therefore greatly reduce the timing overhead for high resolution imagers. In addition, over integration periods are avoided leading to reduced power consumption.

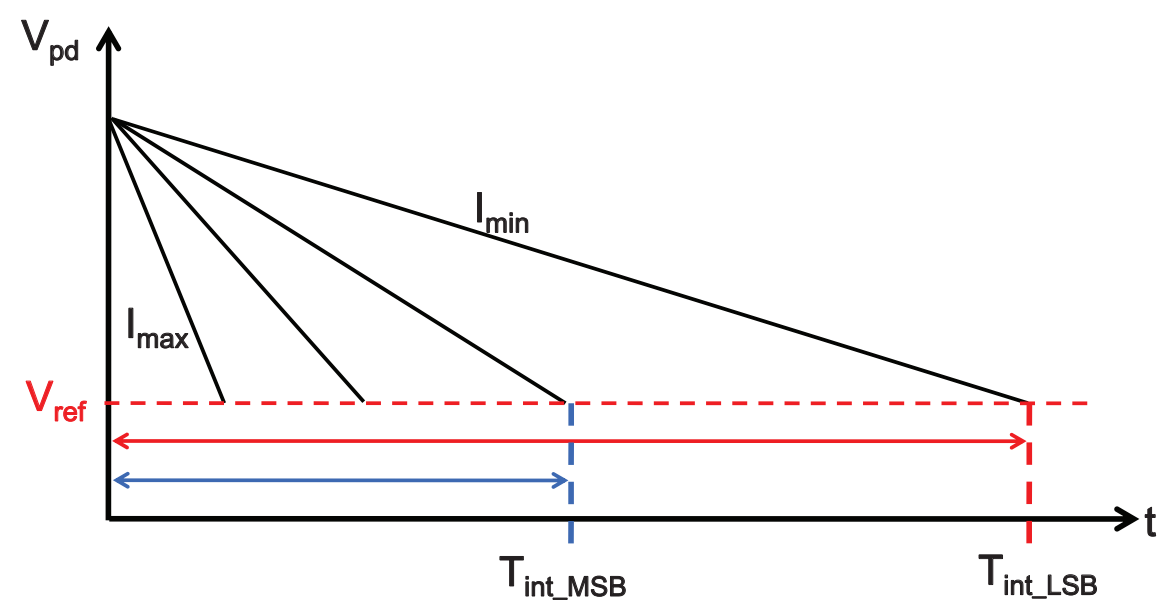

Figure 4.8: Illustration of timing boundaries for a 2-bit resolution.

\subsubsection{Trade-off Analysis}

Depending on the memory size integrated at the pixel level, different area-delay trade-offs can be achieved. Indeed a trade-off can be made between the required number of bits for the pixel-level memory and the required number of reset of the integration phases. MATLAB simulations were performed to extract the trends in pixel area versus the size of the memory embedded at the pixel-level and the results are shown in Figure 4.9. In the simulation, a square pixel with $30 \%$ fillfactor is assumed. The area is estimated for CMOS $0.35 \mu m$ technology. Area limited by the metal wires and the total size of the transistors are also considered. Area of pixel-level embedded memories with 6T SRAM and 2T DRAM are both considered. The figure illustrates that the area of the pixel with embedded $6 \mathrm{~T}$ 
SRAM is linear to the number of embedded bits, while the area of the pixel with embedded 2T DRAM is mainly constrained by metal wires. The size of a pixel using 4 bit 2 TDRAM is only $72 \%$ of the pixel using 4 bit 6 T SRAM.

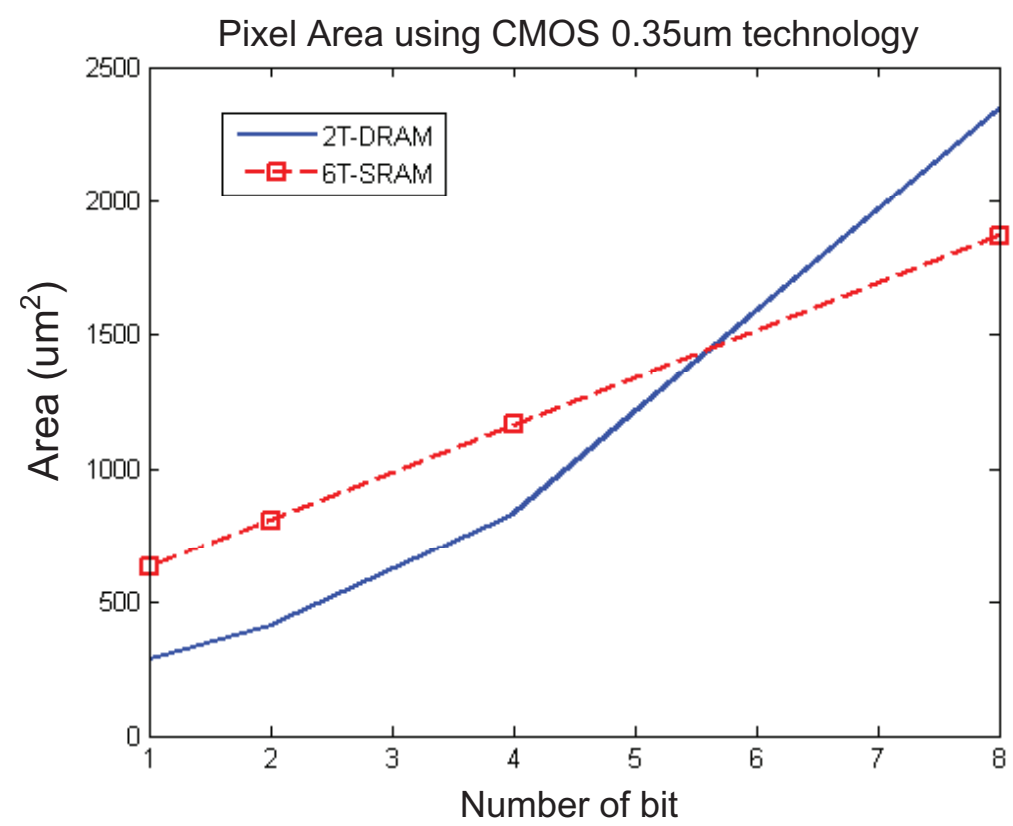

Figure 4.9: Pixel area as function of the number of embedded bits.

MATLAB simulations were also performed to extract the trends in the delay overhead versus the size of the memory embedded at the pixel-level. Figure 4.10 shows the interpolated curve of the delay overhead in terms of integration time versus the memory size $\mathrm{n}$ for an 8-bit resolution by assuming $\frac{I_{\max }}{I_{\min }}=250$. From these results, it appears that using a 4-bit pixel-level memory requires only one reset and leads to less than $10 \%$ overhead on the total integration time. Considering both the area and the integration time overheads, using the 4 bits 2T DRAM optimally maintains a small pixel area and keeps a relatively high operation speed.

\subsubsection{Overall Architecture}

In order to implement the MRI scheme with a dynamic memory, several changes are required compared to the conventional architecture. As a result of the tradeoffs analysis presented in the previous section, the choice to implement a 4 bit memory element has been made. Besides, on top of cutting the memory require- 


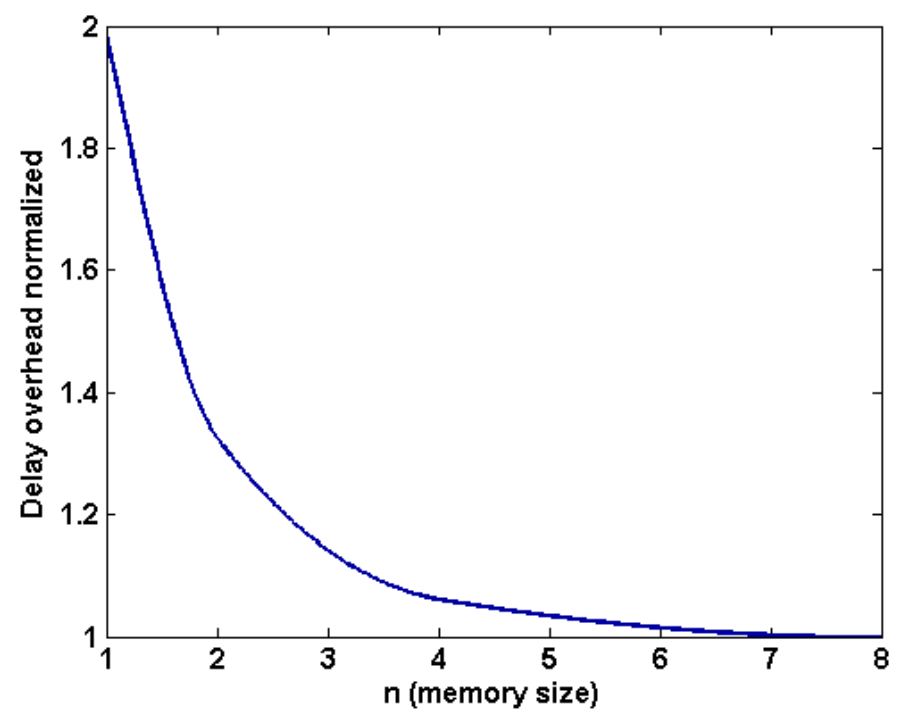

Figure 4.10: Interpolated curve of the delay as a function of memory size.

ments by a factor of two with the MRI scheme, the use of the full CMOS 2T DRAM instead of the $6 \mathrm{~T}$ SRAM cell is proposed to further increase the area savings. The block diagram of the resulting global architecture is presented in Figure 4.11. Two scan shift registers are used to select each line of the array during the reading operation and to shift the read values out of the sensor. The memory sensing and refresh circuit are composed of sense amplifiers to detect the state of the pixel: "fired" or "not fired", as well as to read the content of the pixel-level memories, simultaneously. Based on the state of the pixel, a conditional refresh circuit allows to selectively rewrite the useful information. In other terms, based on an external leakage monitor or an external temperature sensor giving retention time conditions in the memory, the content will be refreshed with minimal overhead for power consumption. A control unit allows the pipeline of the read operation and the scan out operation to minimize the time between two partial integrations. Large write buffers are used to store the information from the global counter inside the pixel-level memory during the integration. A counter timing unit and a linearization circuit are also used to provide the data to the pixel-level memories. 


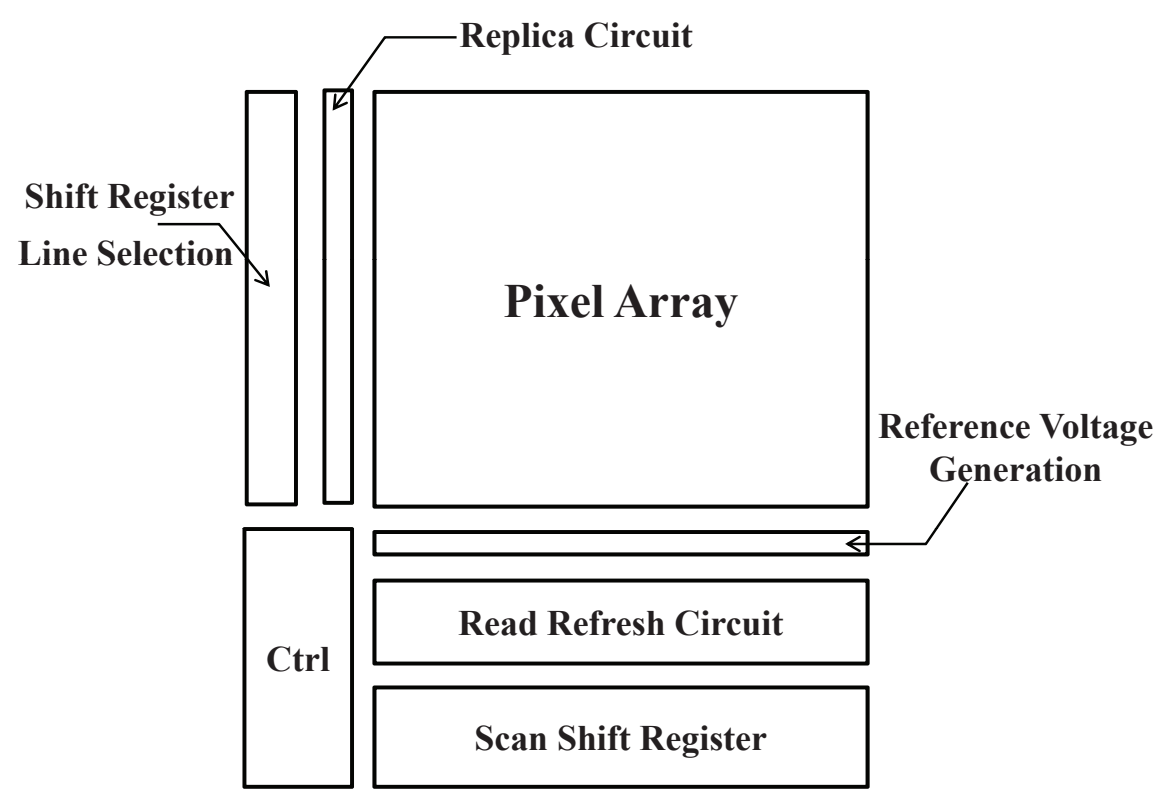

Figure 4.11: Block diagram of the overall architecture.

\subsubsection{Pixel Circuit}

The proposed pixel circuitry is depicted in Figure 4.12, which contains a reset transistor controlled by a global reset signal, a voltage comparator, a flag circuitry to indicate the firing state of the pixel, a pass logic circuit to bypass the output of the comparator during the refresh operation and finally the 2T DRAM cells. The operation of the proposed pixel starts by a reset low signal enabling to set the photodiode voltage to the supply level. At the end of the reset phase, the photodiode is left floating and the voltage of the equivalent capacitance of the photodiode is discharged by the illumination dependant photocurrent. At the same time, a global down counter outside the pixel array is enabled and its data is fed to the internal storage elements of the pixel. The memory is set in the write mode during the integration phase, as shown in Figure 4.13 and the content of one bit DRAM will track the corresponding data line. When the photodiode voltage crosses the reference voltage, the output of the comparator switches disabling the write signal. When a row is accessed the memory is set in the read mode and the flag signal allows the write driver of the column to refresh the data if the pixel has fired. At the same time, all the pixels of the same column belonging to different rows are set in a hold mode in order to prevent a wrong data to be written to the memory. 


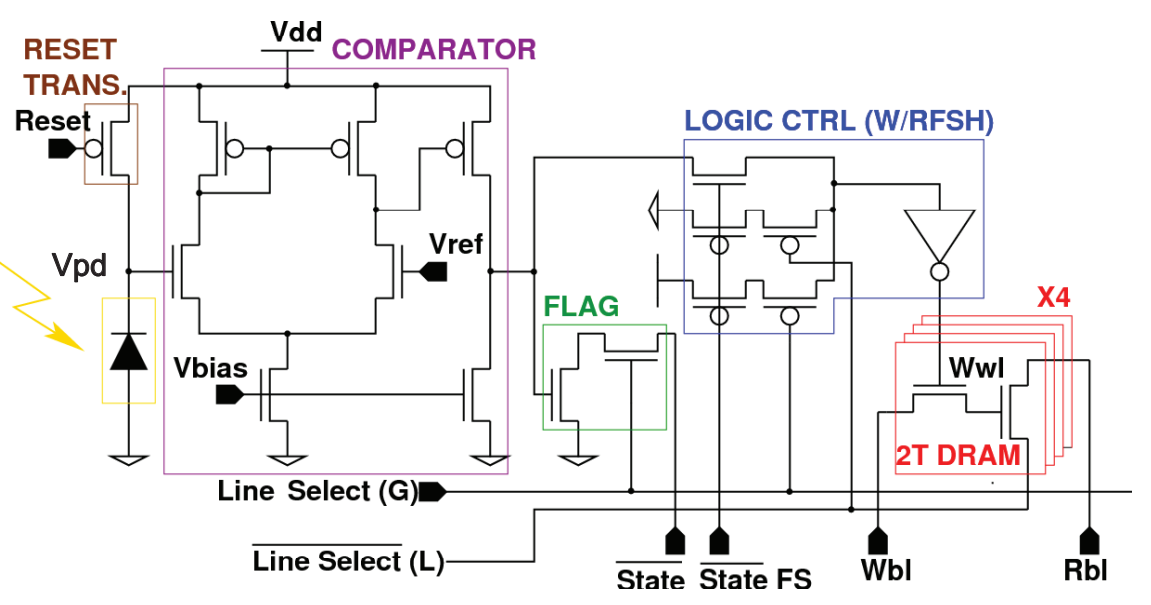

(a) Schematic diagram.

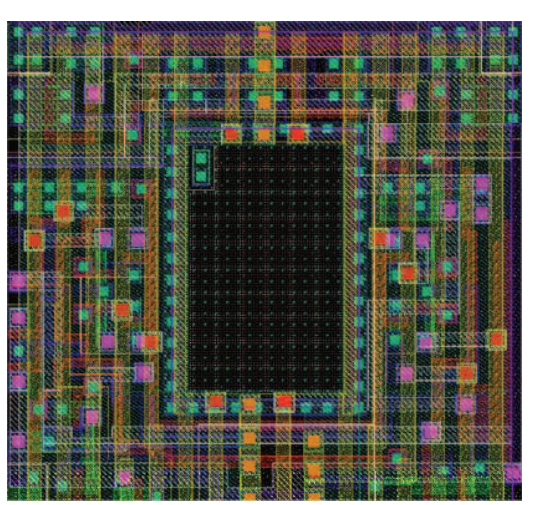

(b) Layout view.

Figure 4.12: Pixel schematic and layout.

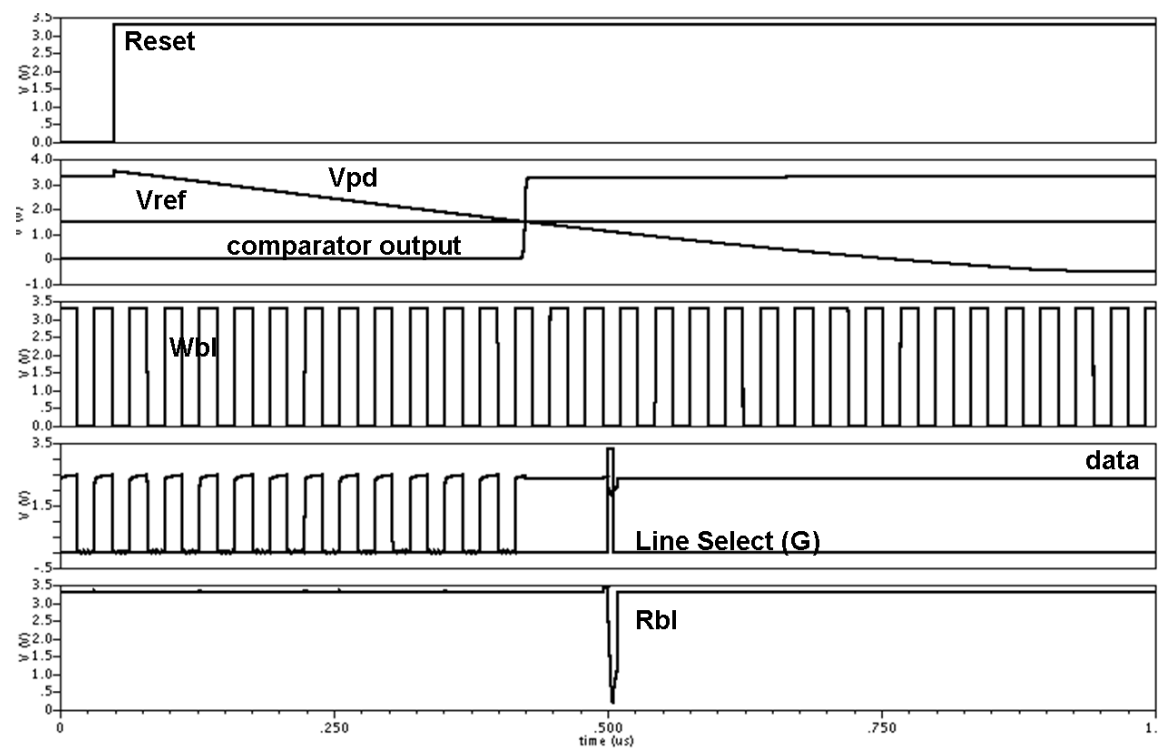

Figure 4.13: Simulated timing diagram illustrating the pixel's operation. 


\subsubsection{T DRAM Implementation}

In the proposed architecture, a 4-bit 2T DRAM is used as the pixel level memory element. The use of 2T-DRAM reduces the area compared to the conventional $6 \mathrm{~T}$ SRAM. The use of a dynamic memory fits very well the requirements of a DPS as a frequent use of the memory is required with multi-reset integration scheme. However, the use of dynamic memories adds some complexity compared to static ones mainly due to the loss of charges on the storage node caused by leakage currents. A refresh circuit is therefore required to solve this problem, depending on external conditions, temperature, supply voltage and process variations. In this part, the implementation of the pixel dedicated memory is described. Some techniques employed in memory design will be reviewed and adapted to the need of the DPS architecture.

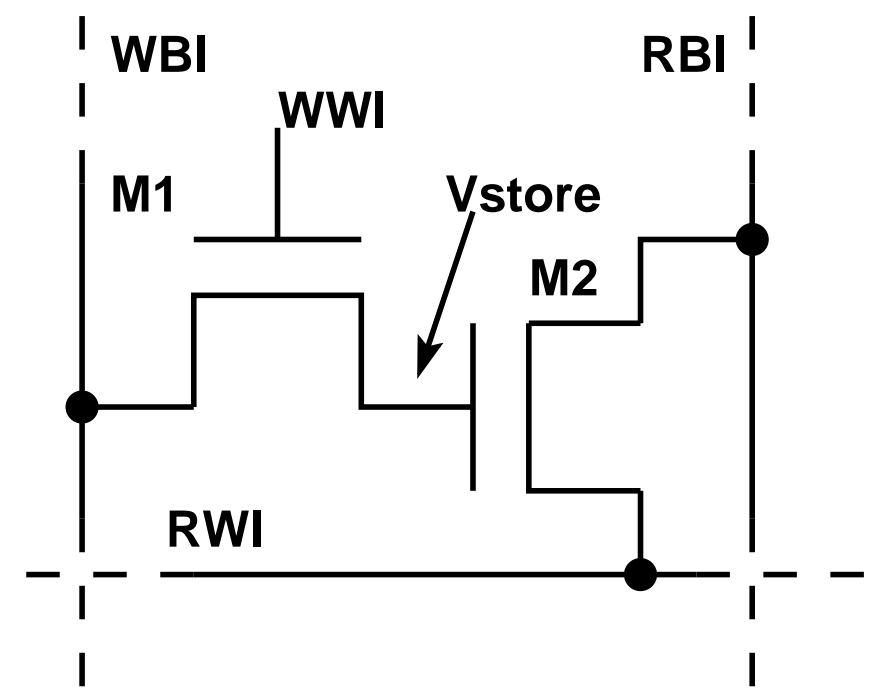

Figure 4.14: 2T-DRAM cell [38].

The 2T DRAM cell shown in Figure 4.14 is derived from the well known 3TDRAM and was proposed recently as a potential memory cell for microprocessor's cache [38]. The main advantage of this memory cell is that it uses a full CMOS technology and it improves density compared to the 3T-DRAM, by removing the access transistor. The operating principle illustrated in Figure 4.15 can be summarized as follows: during a write operation, the write-bit-line is set high or low in order to charge or discharge the storage node loaded by the gate capacitance of transistor M2 and the diffusion capacitance of transistor M1. Note that for a 


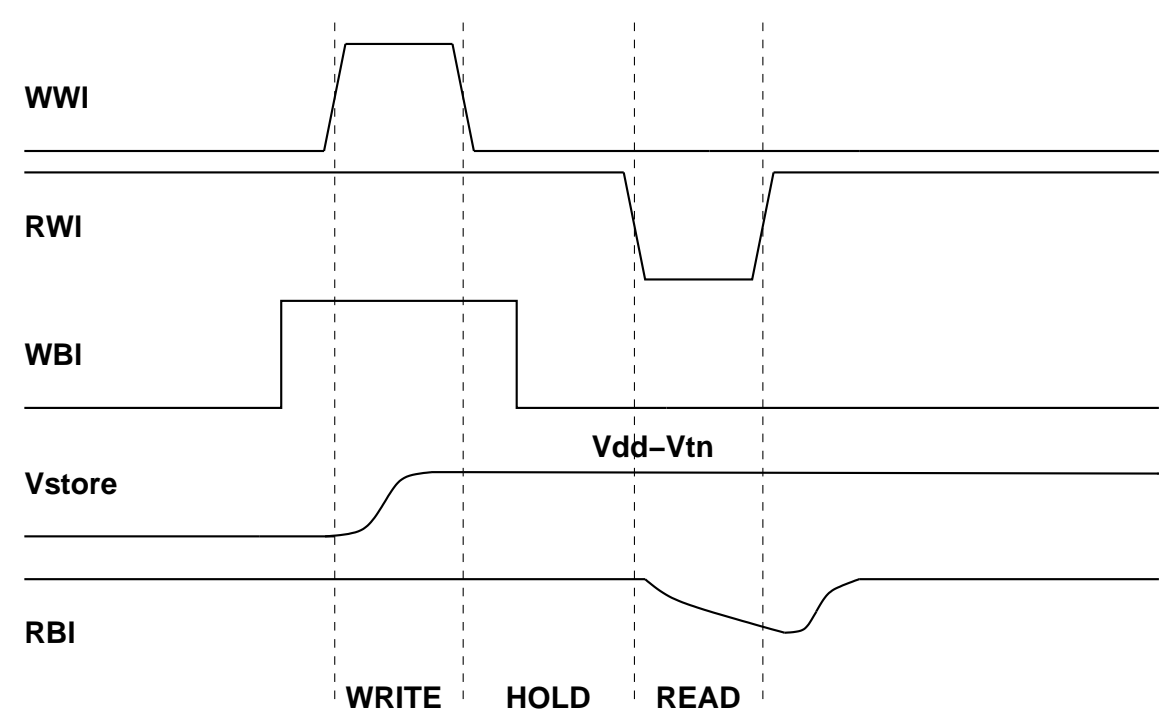

Figure 4.15: 2T-DRAM cell Operating principle.

conventional NMOS bit-cell, the gate voltage for storing the state 1 will be limited to the supply voltage minus one threshold voltage. In the hold mode, the write word-line is kept low and the leakage currents discharge progressively the internal node and therefore determine the data retention time and correspondingly the required refresh period. During the read operation, the read word-line is set low enabling or disabling the discharge of the pre-charged read bit-line, depending on the stored state on the gate of transistor $M 2$.

\subsubsection{Differential sensing scheme and voltage generation}

The choice of differential sensing was made as it reduces the voltage swing on the bit-lines and thus the power consumption incurred by the read operation. Besides, differential sensing improves the robustness to common mode noise on the bit-line such as supply voltage variations. This scheme has however a cost as it requires both a bit-line and a reference bit-line for a single cell and a reference voltage generation circuit. In order to reduce coupling noise between bit-lines, transposed bit-line architecture is adopted using regular interleave of bit-lines within the pixel array. As shown in Figure 4.16, each column is divided into subblocks indexed either "odd" or "even". During a read operation, the control unit outputs the state of the block being accessed either "odd" or "even". This signal allows to determine the bit-line and the reference bit-line for correct reference 
voltage generation and also to configure the latch input, using a multiplexer. The reference voltage is generated using a row of dummy cells either stuck at value 0 or 1 , giving an intermediate voltage for the sensing operation.

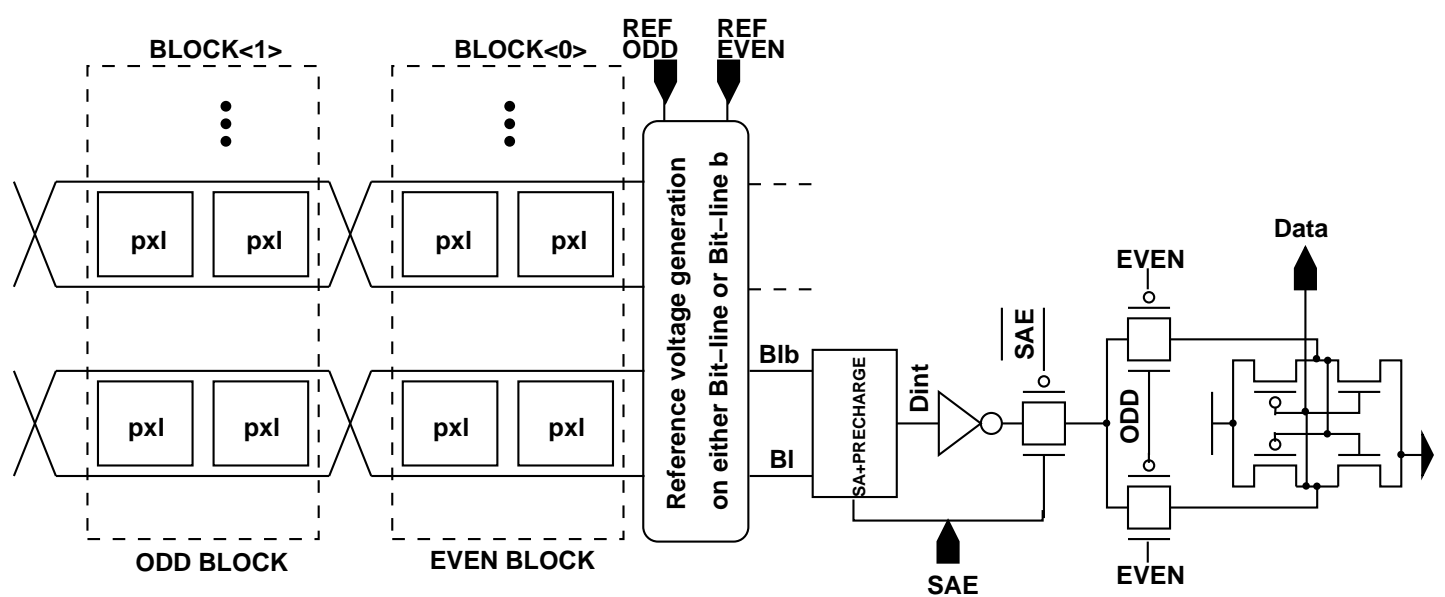

Figure 4.16: Diagram of the transposed bit-line architecture and the latching stage.

\subsubsection{Read and Refresh Circuits}

In order to activate the sense amplifier at the right time for correct sensing of the memory cell, a replica circuit (Figure 4.17) based on a dummy column and a dummy memory cell is used [39]. Note that for correct sensing of the memory, the differential voltage, i.e., the voltage between the reference bit-line and the bit-line to be read, must be higher than the offset value of the sense amplifier. Figure 4.19 depicts the reading stage designed to access the pixel level memory. As all memory cells of a same pixel must be read simultaneously, a simple sense amplifier structure was chosen. A basic latch-based sense amplifier is used to fit within the pixel pitch. Each pixel contains 4 bits, all are read simultaneously. Therefore, four sense amplifiers have to fit with one column pitch. The reading operation is a large contributor to the total power consumption of the chip. Therefore the charge required during pre-charge operation of the large bit-line capacitances and the large static current flowing through the sense amplifier must be reduced as much as possible. As a consequence, a close control of the sense amplifier timing is critical and can reduce large amount of power. As the sensing operation is differential, the generation of a reference voltage is required. The reference voltage 
is generated as in [38], as shown in Fig 4.18. The transposed bit-line architecture allows to reduce the coupling effects between two adjacent lines but adds some complexity. In order to keep power under control, the refresh operation is performed only on the fired pixel. This prevents unnecessary switching transitions on the data buses, thus saves power. In order to achieve this, a flag signal is used to detect the state of the pixel and only if the pixel has fired the content of the memory is read and refreshed. To control the refresh operation a signal is fed to the DPS starting the refresh operation. Fig.4.20 shows the simulated curves of the memory read and conditional refresh operation. In the sense amplifying period, only after the sense-amplify-enable (Sae) signal becomes valid, the difference of the bit line (BI) and the reference bit line (Refbl) is sensed and used for updating the pixel's value. If the pixel is fired, the new value (Data) will be put on the write bit line (Wbl) through the 2-1 MUX via the control of the state signal.

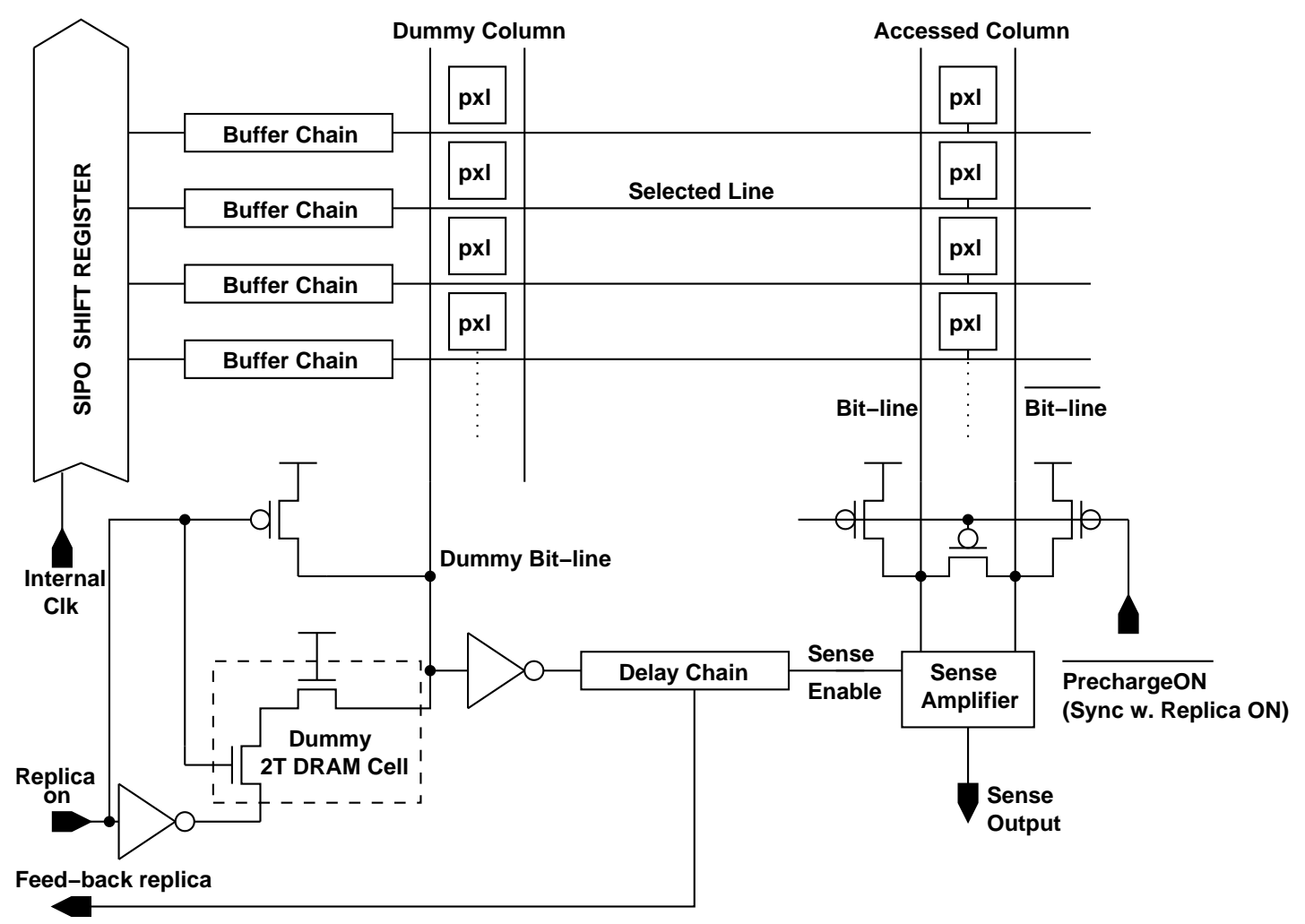

Figure 4.17: Schematic view of the replica scheme. 


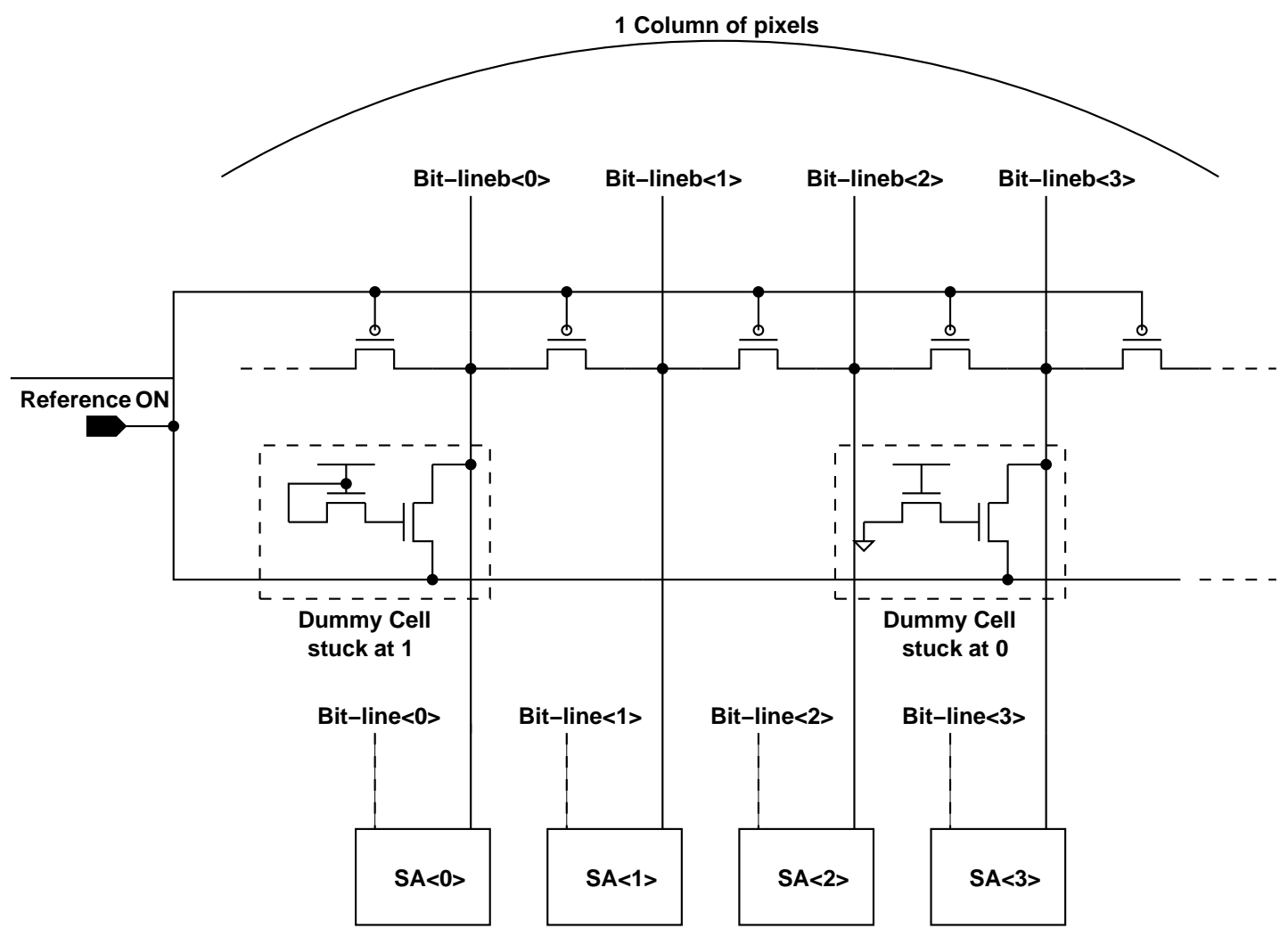

Reference voltage generated with average of 64 cells stuck at and 64 cells stuck at 0

Figure 4.18: Diagram of the reference voltage generation [38].

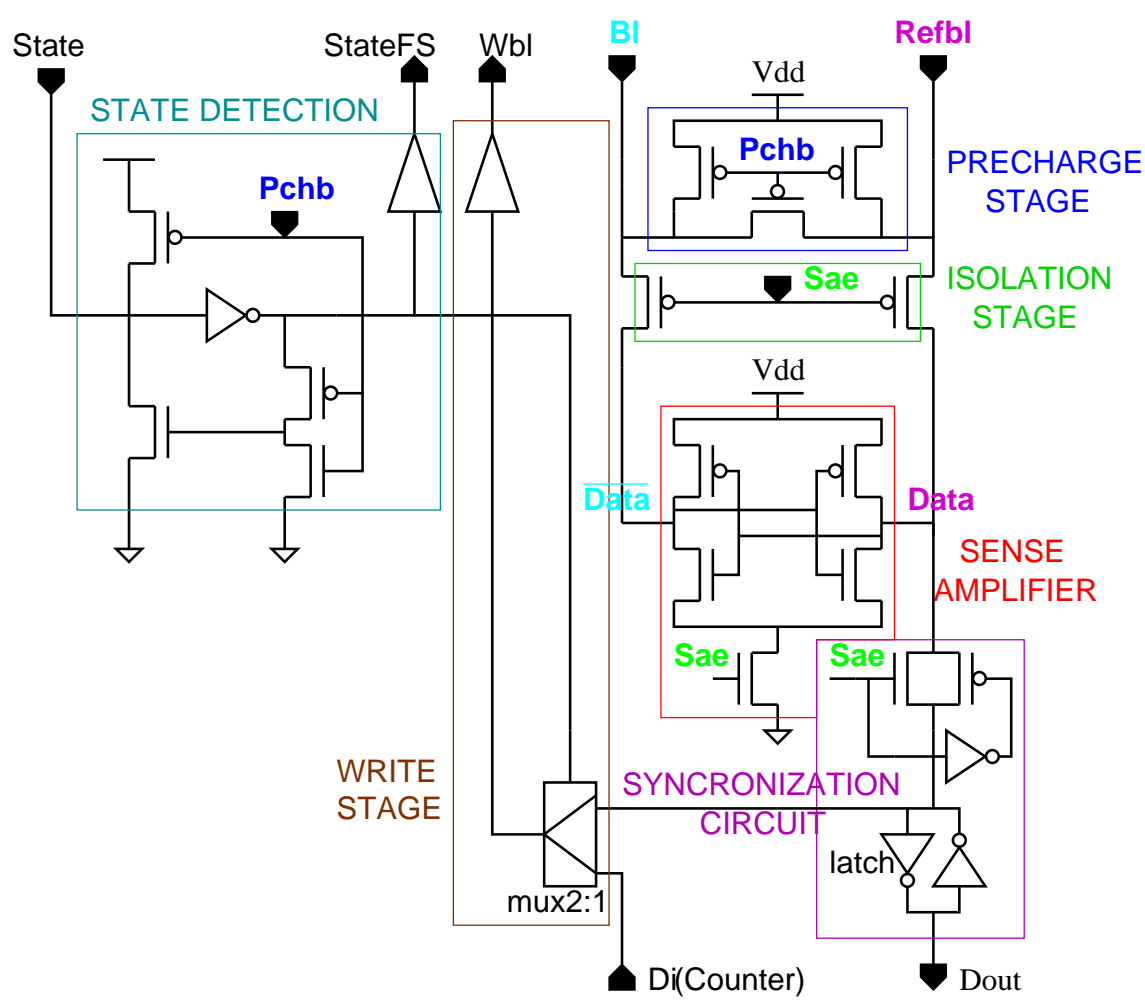

Figure 4.19: Schematic diagram of the memory read and refresh scheme. 


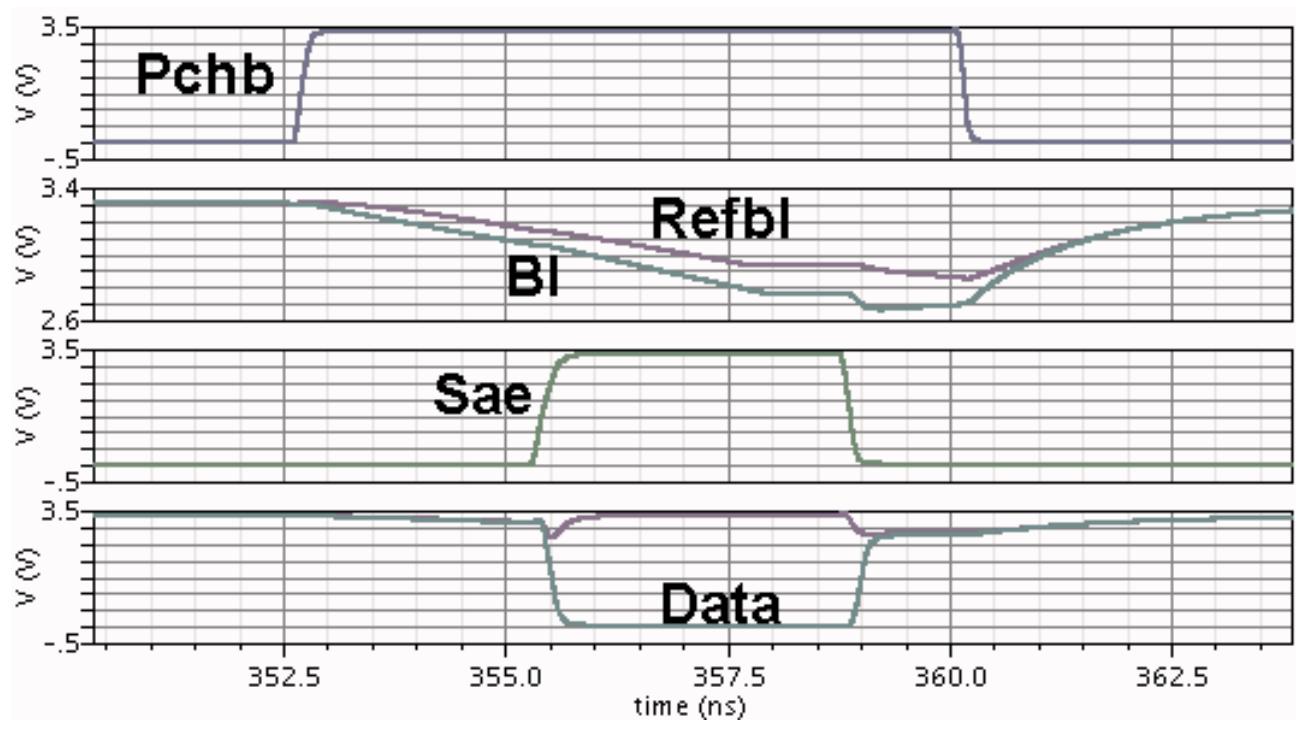

Figure 4.20: Simulated Curves.

\subsection{Power Analysis and Power Reduction Tech- niques}

In this part, an analysis of the power consumption of the proposed architecture is given. Power consumption results were obtained from electrical simulations using Spectre simulator from Cadence. The aim of this analysis is to identify the major contributors to the total power consumption by giving the distribution of total power among different blocks of the architecture such as the array, the sense amplifier, precharge circuit and the write buffers. This analysis is then used to consider power reduction techniques that are generally costly in terms of speed and area, only on critical blocks of the architecture.

\subsubsection{Power Consumption Analysis}

In order to perform our power consumption analysis, a critical path of the $64 \times 64$ DPS has been designed and simulated using Spectre electrical simulator to reduce the netlist size and therefore the simulation time. From this analysis, it is clear that main contributor to power and energy consumptions are the pixels since the voltage comparator of each pixel is drawing large static current during the whole integration time. Considering mobile applications, energy is the key metric to 
be considered and except the energy required by the pixel to capture one frame, one can say that the remaining energy consumption is spread on all blocks. The energy consumption from scan shift registers can be neglected. Table 4.1 shows the power consumption analysis from electrical simulation at nominal process and supply voltage.

\begin{tabular}{|c|c|c|}
\hline & $\begin{array}{c}\text { Average power } \\
\text { at } 30 \mathrm{f} / \mathrm{s}\end{array}$ & $\begin{array}{c}\text { Average energy per frame } \\
\text { at } 30 \mathrm{f} / \mathrm{s}\end{array}$ \\
\hline Array (Pixels) & $13.9 \mathrm{~mW}$ & $13.9 \mathrm{uJ}(3.4 \mathrm{~nJ} /$ pixel $)$ \\
\hline Scan shift register & $800 \mathrm{nW}$ & $23 \mathrm{~nJ}$ \\
\hline Write buffers & $24 \mathrm{uW}$ & $24 \mathrm{~nJ}$ \\
\hline Scan register for line selection & $10 \mathrm{nW}$ & $0.3 \mathrm{~nJ}$ \\
\hline Precharge and sense amplifiers & $430 \mathrm{nW}$ & $13 \mathrm{~nJ}$ \\
\hline
\end{tabular}

Table 4.1: Power consumption analysis from electrical simulation at nominal process and supply voltage.

\subsubsection{Power Reduction Techniques}

The power consumed by the voltage comparators contained in each pixel represents the major contributor of the total power consumption. In order to reduce the power consumption compared to the actual implementation, either an auto-reset function employing a feedback unit or a different comparator structure could be used. The main advantage of a latch-based sense amplifier is the power consumption reduction compared to the conventional architecture, where a bias current is drawn permanently from the supply. However, this kind of comparator suffers from large offset resulting in a lower image quality. To solve this problem, a pre-amplifier may be used as a first stage at the cost of increased area overhead. Another solution is the use of switched op-amp technique, allowing to enable or disable the comparator using a clock signal. This circuit matches well with the time domain DPS architecture. Indeed, during the analog to digital conversion, the voltage of the photodiode needs to be compared to a reference voltage $V_{\text {ref }}$ periodically when the global counter output is switching from one state to another. If the global counter frequency is low enough to switch on the comparator during a short period before the counter value changes, then power savings may be achieved. This scheme is even more efficient when using the non-uniform 
quantization scheme, as the frequency is reduced along with the integration period. Therefore the switching activity and the time during which the comparator is on is strongly reduced compared with the standard implementation. Figure 4.21 depicts the schematic of the proposed implementation for the DPS voltage comparator and simulated curves of key signals. Compared with the conventional pixel level comparator, 2 PMOS and 1 NMOS are added to control the switching. These three gates only increase the size of the proposed design in Figure 4.12 by $2.8 \%$. Transistors MN1-MN2-MP1-MP2 constitute the differential pair, MNB is a bias transistor with its gate voltage controlled externally for improved flexibility. MNF is a footer transistor to cut the supply path once the pixel is fired. MP3 and MN3 constitute the output stage of the comparator to have a full swing signal output voltage os2. MPS is added to set the correct state on the output during inactive mode by controlling the voltage os1. Note that we can use this comparator with a photodiode set in photovoltaic mode allowing for energy harvesting capabilities. The feedback circuit used to disable the operation of the comparator is not affecting the reset transistor but controlling the supply path and enabling to maximize the time during which the photodiode is in the energy harvesting mode. From the simulation results (Figure. 4.22), one can observe that while enabling periodically the comparator, only one pulse is observed on signal os2 corresponding to the crossing of the photodiode voltage with the voltage level $V_{\text {ref }}$. The simulated power consumption of the voltage comparator is in the range of $\mathrm{nW}$ depending on the frequency and linearization scheme used, which greatly reduces the power consumption of the original implementation by 2 to 3 orders of magnitude.

\subsection{Hardware and Measurement Results}

In order to validate the proposed architecture, a prototype of a $64 \times 64$ DPS array was designed using the AMS $0.35 \mu \mathrm{m}$ CMOS technology. Figure 4.23 shows the microphotograph of the fabricated chip illustrating the different parts of the chip namely, the pixel array, the shift registers for line selection and scan-out of data as well as the control block. 


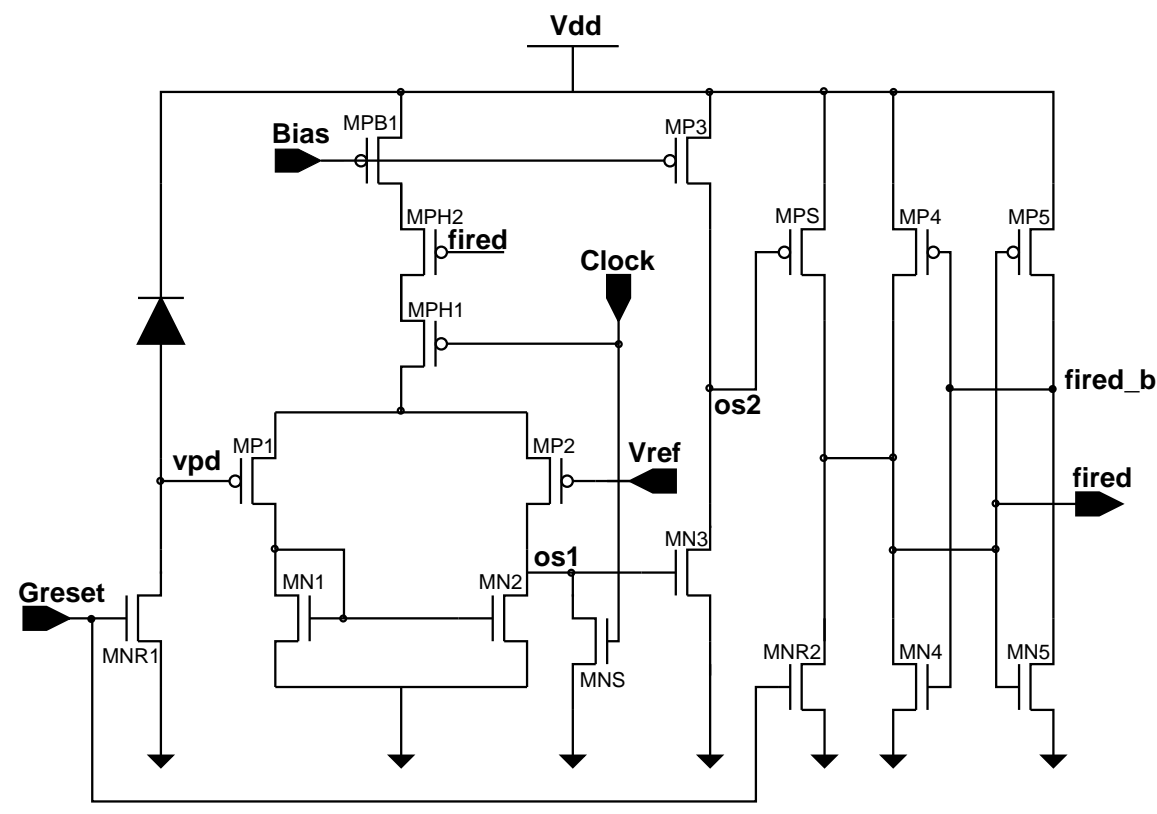

Figure 4.21: Schematic diagram of the proposed switched-opamp comparator for PWM DPS applications.

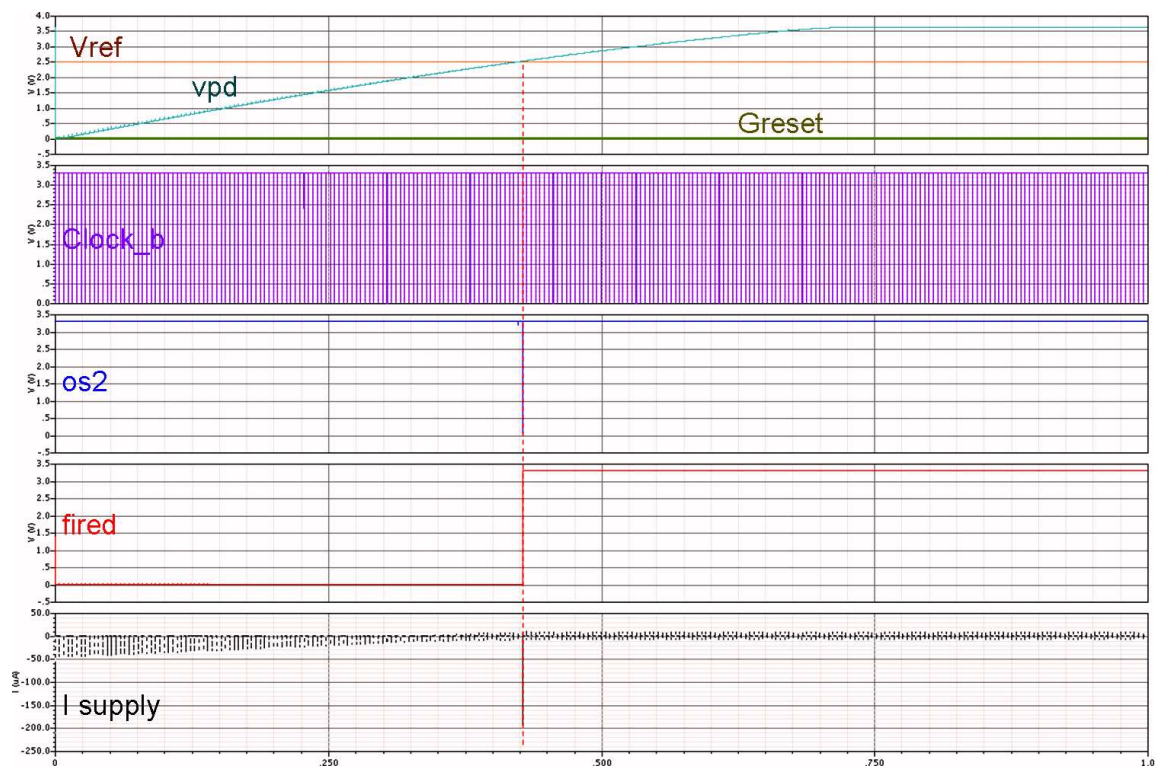

Figure 4.22: Simulation results at nominal process and supply. 


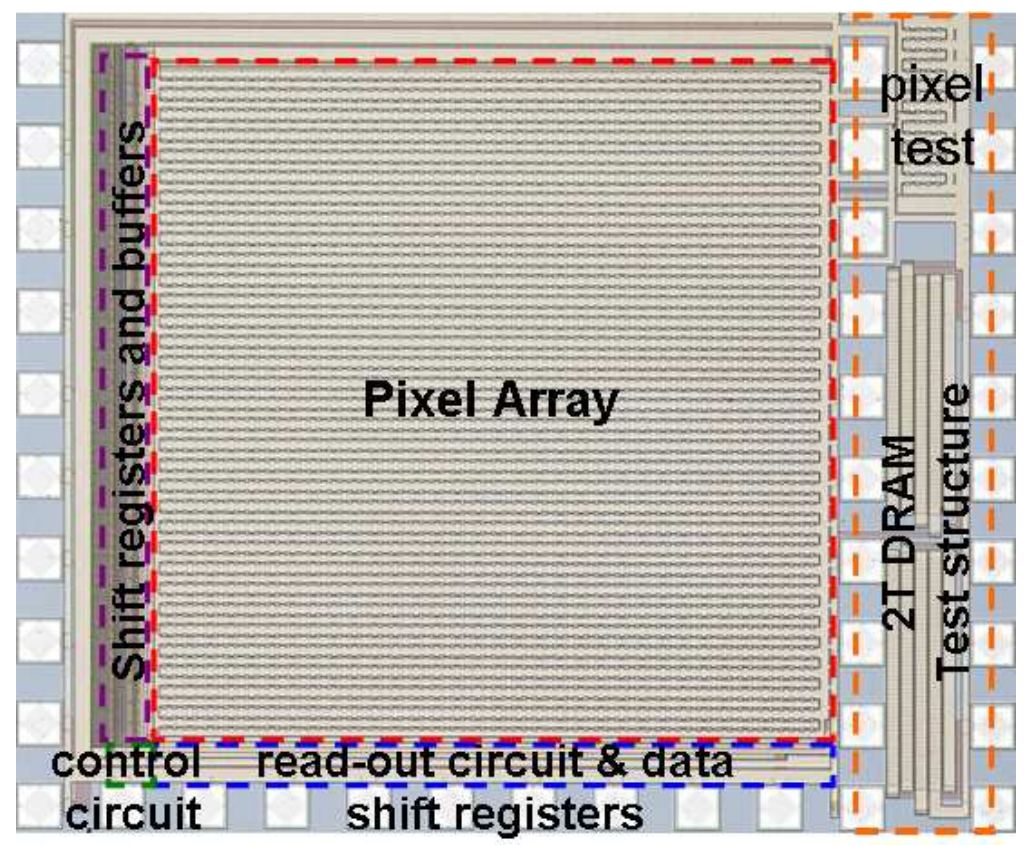

Figure 4.23: Microphotograph of the prototype.

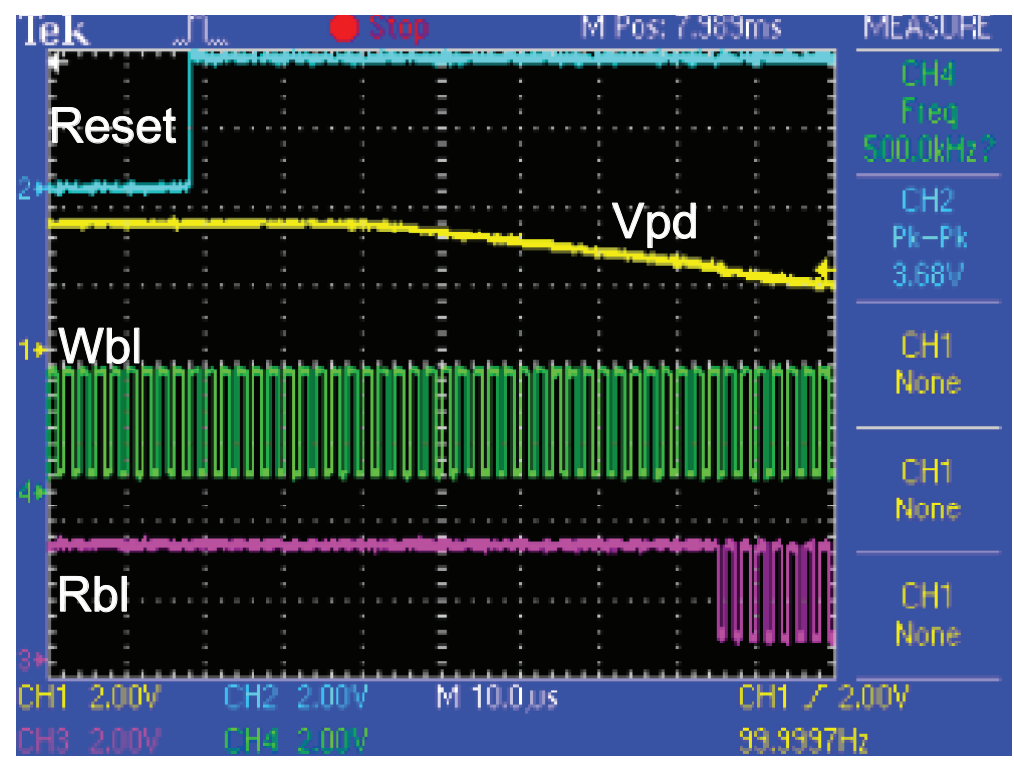

Figure 4.24: Measurement results of key electrical signals. Reset, Vpd, Wbl and $\mathrm{Rbl}$ are the reset signal, the photodiode voltage, the right bit line signal and the read bit line signal, respectively. 
The functionality of the DPS using MRI concept was validated and successfully tested. Electrical signals from the pixel were successfully captured. Figure 4.24 illustrates the signals captured within the pixel using a Tektronix DS2024 oscilloscope. This figure shows electrical signal both inside and outside the pixel, to demonstrate its functionality. Top to bottom signals illustrated in Figure 4.24 correspond to the complement of the reset signal, the photodiode node voltage, the line selection signal and the complement of the flag state signal after the read-out phase. It is clearly illustrated that once the pixel is reset the photodiode voltage starts to decrease due to the photo-generated current. While sampling periodically the output of the comparator, the complement of the state signal remains high until the photodiode voltage crosses the reference voltage.

Table 4.2 summarizes the main characteristics of the proposed implementation compared to previous 6-T SRAM based DPS implementations [36] and [31] using the same technology node. The area of the proposed pixel is $22 \mu \mathrm{m} \times 22 \mu \mathrm{m}$ and the fill factor is $20 \%$, which could be further improved with the use of a single ended sensing scheme compared to the differential one we used in this work. While the current consumption per pixel is improved compared to previous implementations, it is expected that further benefits can be obtained using power reduction techniques discussed in previous sections.

\begin{tabular}{|c|c|c|c|c|}
\hline & This work & {$[36]$} & {$[31]$ (conventional) } & {$[33]$} \\
\hline Technology & $0.35 \mu \mathrm{m}$ & $0.35 \mu \mathrm{m}$ & $0.35 \mu \mathrm{m}$ & $0.35 \mu \mathrm{m}$ \\
\hline Supply voltage & $3.3 \mathrm{~V}$ & $3.3 \mathrm{~V}$ & $3.3 \mathrm{~V}$ & $3.3 \mathrm{~V}$ \\
\hline Pixel area & $22 \mu \mathrm{m} \times 22 \mu \mathrm{m}$ & $30 \mu \mathrm{m} \times 26 \mu \mathrm{m}$ & $45 \mu \mathrm{m} \times 45 \mu \mathrm{m}$ & $50 \mu \mathrm{m} \times 50 \mu \mathrm{m}$ \\
\hline Fill factor & $20 \%$ & $16 \%$ & $12 \%$ & $20 \%$ \\
\hline Pixel current & $1 \mu \mathrm{A}$ & N/A & $1.6 \mu \mathrm{A}$ & N $/ \mathrm{A}$ \\
\hline Transistor count & 25 & 32 & 91 & 38 \\
\hline Resolution & $4 / 8 \mathrm{bits}$ & $4 / 8 \mathrm{bits}$ & $8 \mathrm{bits}$ & $4 / 8 \mathrm{bits}$ \\
\hline Frame/second & 33 & 300 & $33^{*}$ & $33^{*}$ \\
\hline
\end{tabular}

*: estimated from the corresponding literature.

Table 4.2: Comparison of key metrics with related work. 


\subsection{Summary}

Digital pixel sensors are promising architectures for high speed image acquisition, high dynamic range and high illumination image. However, the area occupied by the memory is a major drawback reducing the pixel sensitivity to light. In this chapter two different approaches were explored to reduce the area of the pixel memory. The first approach reduces the memory requirements by using a multireset integration scheme. The second approach directly improves the area of the storage element using a 2T-DRAM cell instead of the area-consuming $6 \mathrm{~T}$ SRAM cell. To successfully implement this concept, a new DPS architecture was proposed. This DPS relies on a multi-reset integration scheme that takes benefit from the chronological way the bits of the code are changing. Using this scheme, a four bit per pixel memory was employed making the design of $20 \%$ fill factor and $22 \mu \mathrm{m} \times 22 \mu \mathrm{m}$ digital pixel sensor possible. Considering the DPS architecture for mobile applications, the power consumption of the sensor is also of major concern. Detailed analysis of the power contributors presented in this chapter helped to identify the main building blocks contributing to power. The voltage comparator within each pixel is identified as the bottleneck in terms of power due to its large static current during the whole integration phase. Several power reduction techniques for future DPS implementations were also proposed and discussed in this chapter. 


\section{Chapter 5}

\section{Bit-Plane Based Hough}

\section{Transform Processing for Real-Time Image Segmentation}

\subsection{Introduction}

A gray-scale image can be decomposed into a series of binary images:

$$
p^{t}(i, j)=a_{k-1} 2^{(k-1)}+a_{k-2} 2^{(k-2)}+\ldots a_{1} 2^{1}+a_{0} 2^{0}
$$

A bit plane of a digital image is a set of bits having the same position in the respective binary numbers. For example, for 8 -bit data representation there are 8 bit-planes: the first bit plane contains the set of the most significant bit and the 8th contains the least significant bit. It is possible to see that the first bit-plane gives the roughest but the most critical approximation of outlines of an image, and the lower the number of the bit plane, the more is its contribution to the detailed information, referring to Fig.5.1.

The advantages of bit plane based processing are:

1. Memory space for storing images is highly reduced since the image can be 


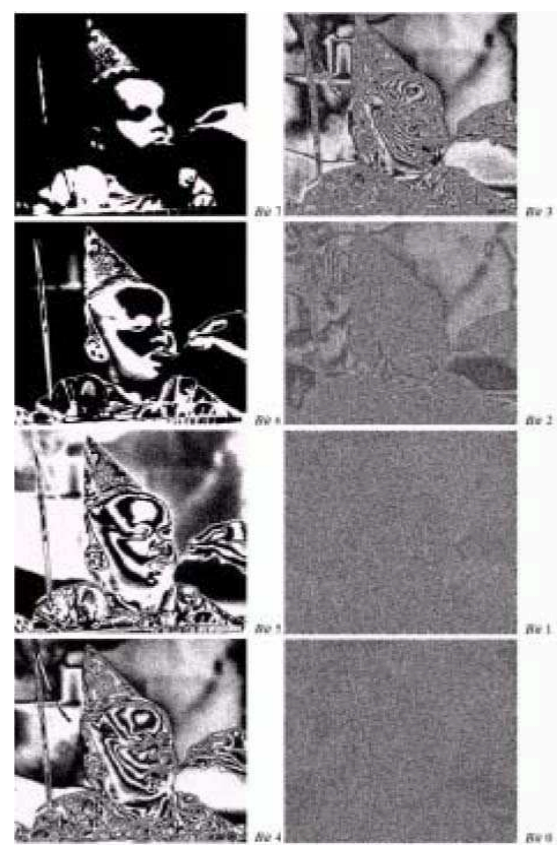

Figure 5.1: The four most significant binary (left column) and four least significant binary (right column) bit planes of an image [40].

written in binary formats.

2. The bit planes naturally separate the features of the image. They are able to eliminate some features, whereas highlight the others. For example, in Fig.5.1, bit plane 7 highlights the outline of the baby, whereas the bit plane 0 eliminates all the shape information and just shows some imperceptible details.

3. Bit planes help to highly reduce the computational complexity of digital image processing, because they turn all of the arithmetic operations to Boolean operations.

4. Bit plane technique can be extended to true RGB color images easily, and it is similarly easy to get back to a gray scale image or color image.

There are many applications based on bit plane digital image processing. [41] developed a correlated bit plane model for Chinese materia medica starch grains classification. The model is shown to match well with the observed histogram$\mathrm{s}$ for texture and natural images, and performs better than the state-of-the-art distributions. [42] proposed a lossless coding scheme for images by using cross 
point regions on multiple bit planes. A meaningful improvement in compression ratio has been obtained, compared to other authors' relative works. In [43], a novel method of crowd estimation in public locations is proposed, in which bit plane neural network is used for training and constructing classification model. The method is proved to have stronger robustness and real-time feature, and suitable for many kinds of complex locations. [44] proposed a digital image stabilization scheme based on the block matching technique using the bit plane images. The proposed motion estimation process can be implemented using only binary boolean functions, which have significantly reduced computational complexity. Experimental results demonstrate that the proposed scheme can provide good performance comparable with that of conventional algorithms. [45] used bit plane based decomposition approach as a diversification method to implement the handwriting recognition system. Bit plane approach helps to address the huge memory space requirement and intensive computation problems, hence enables to combine a pool of classifiers to show complementary decisions and higher performance.

In [46], a new architecture for color space conversion in RGB to YCbCr domain has been proposed. The architecture exploits the similarity in bit planes of a natural images to bring the algorithmic efficiency of distributed arithmetic (DA) approach close to that of a full multiplier design. Modifications have been carried out in DA to further reduce the computational complexity by a factor of two for most natural images. [47] developed a technique for iris pattern extraction utilizing the least significant bit plane: the least significant bit of every pixel in the image. Through binary morphology applied to the bit plane the pupillary boundary of the iris is determined. Iris pattern extraction is not dependent on circular edge detection, which allows for an expanded functionality of iris identification technology by no longer requiring a frontal view. In [48], a new approach for image coding based on bit plane decomposition and binary morphological operations is presented. The image is first processed by an error diffusion technique in order to reduce the number of bit planes without a significant quality degradation. The bit planes of the resulting image are converted to gray-coded and are represented by a modified morphological skeleton. These reduced modified 
morphological skeletons are coded with an entropy coding scheme, which was particularly devised for efficient skeleton coding. [49] proposed a novel approach to make information analysis and reconstruction. Bit plane slicing decomposition is used to observe image features in a simple domain, to analyze information, and to reconstruct missing pixels to restore corrupted zones, respecting boundary conditions. [50] uses SERVE feature to do the image retrieval. Computation of SERVE is simple, fast, and does not involve any floating point operation. It is derived by the Euler number of the partial binary image formed by certain pixel overlap relations among the four most significant bit planes of the gray-tone image. Experimental results show that its is an aggregating feature and the intuitive justification in favor of SERVE lies in its improved capability of capturing connectivity information across the bit planes of a gray-tone images.

\subsection{Hough Transform}

With the development of modern embedded multimedia applications, many image processing tasks needed to be implemented in hardware. Such tasks include image enhancement and object recognition. Given the geometry of an object, we want to identify its pose, scale and position in the image. The human visual system performs this task with little effort, but it has been proven very difficult for machines to perform equally well automatically. Without the priori knowledge of the image, an exhaustive search needs to be performed to identify the object. The percentage of matches between object and image features are interpreted as the likelihood for a respective transformation to be correct. However, to attain reliable matching results, the detection of straight lines and basic shapes (circle, ellipse, triangle, etc) is the primary stage to recognize objects' shapes in the image.

Although many hardware architectures have been reported, efficient, realtime and low-power image processing platforms are unavailable due to their heavy computations requirements. The Hough transform (HT) provides an efficient method of finding basic geometry in an image by extracting global consistencies 
in the image data. With HT, the difficult image pattern detection problem is converted into a local peak detection problem addressed by a voting process [52]. Hough transform can be used to detect many patterns such as lines, circles, ellipses, curves, polygons, corners and shapes. The Hough transform method is attractive because of: a) its accuracy in recognizing gapped or occluded objects in noisy images, b) its ability to detect any shape, and c) its strong implicit parallelism, which is well geared towards hardware implementation. For our system, HT has particular advantages. First, it can be easily decomposed into bit-plane based computations without performance loss. Second, in the voting phase, for each vote received, only one increment operation is needed in our bit-plane based approach. On the contrary, in traditional full-precision HT, the 8-bit pixel values need to be counted in because the pixel intensity needs to be considered.

Fig.5.2 shows the concept of Hough transform for line detection. Consider the general representation of a straight line in slope-intercept form:

$$
y=a x+b
$$

where $\mathrm{x}, \mathrm{y}$ are the coordinates of the point on this line, and a,b are the slope and intercept of the line. However, if we rewrite Eq.(5.2) to Eq.(5.3), the line (a, b) in Fig.5.2 (a) will be characterized as a point (x, y) in the new a-b space, as shown in Fig.5.2 (b). The a-b space is also called the parameter space.

$$
b=-a x+y
$$

In Fig.5.2 (c), a few of lines intersect at point $\left(x_{0}, y_{0}\right)$, which are mapped to different points in the parameter space, seeing Fig.5.2 (c). However, these points will be colinear because every one of them can be represented by Eq.(5.4), where $\left(a_{i}, b_{i}\right)$ are their slopes and intersects in $\mathrm{x}-\mathrm{y}$ space.

$$
b_{i}=-a_{i} x_{0}+y_{0}
$$


Image space

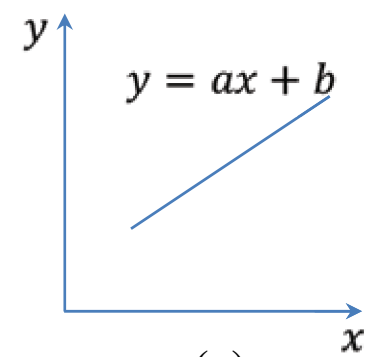

(a)

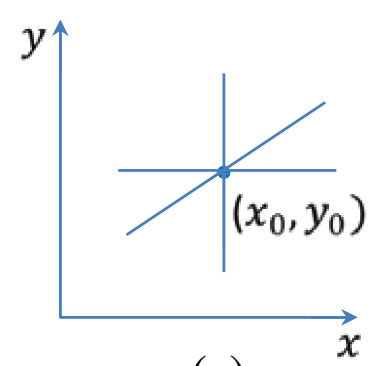

(c)

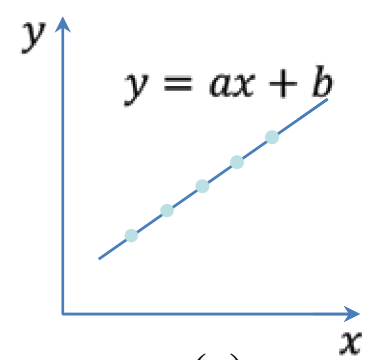

(e)

\section{Parameter space}

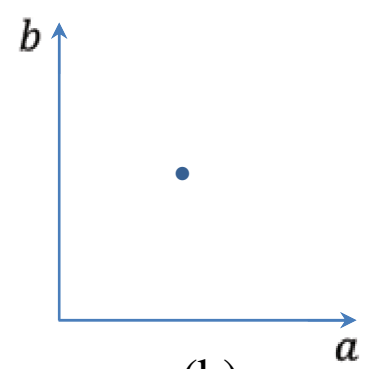

(b)

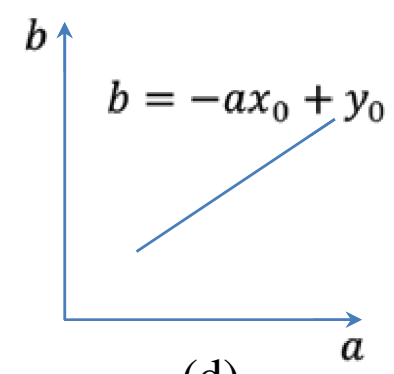

(d)

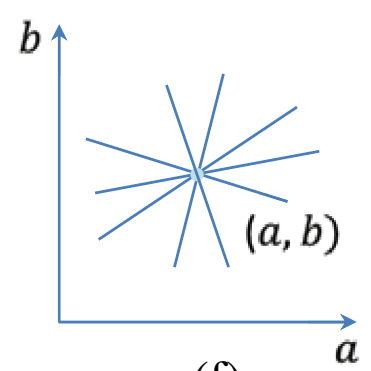

(f)

Figure 5.2: The concept of Hough transform for line detection. 
Therefore, there are some very interesting properties for the transformations of points and lines among the $\mathrm{x}-\mathrm{y}$ space (an image) and the parameter space:

1. A straight line in the $x-y$ space (an image) corresponds to a point in the parameter space, referring to Fig.5.2 (a) and (b).

2. A point in the $x-y$ space (an image) corresponds to a straight line in the parameter space. The coordinates of this point is the slope and intercept of the line in the parameter space, referring to Fig.5.2 (c) and (d).

3. Points which are lying on the same line in $x-y$ space correspond to different lines in the parameter space. However, they intersect at point $(a, b)$, referring to Fig.5.2 (e) and (f).

Thus, the problem of detecting colinear points is converted to the problem of finding concurrent lines. The parameter space is defined to describe lines in the picture plane. However, because both the slope and the intercept can be unbounded, which complicates the implementation of this algorithm, we choose to use an alternative parametrization. As illustrated in Fig.5.3, this parametrization specifies a straight line by the angle $\theta$ of its normal and its algebraic distance $\rho$ from the origin. The equation of this representation is:

$$
\rho=x \cos \theta+y \sin \theta
$$

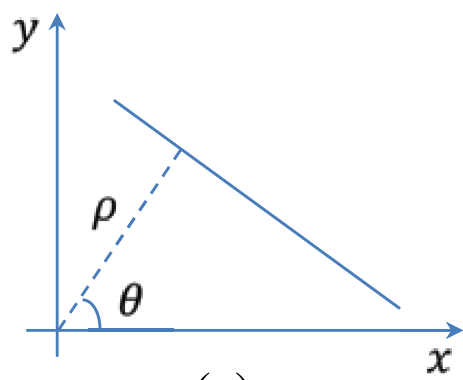

(a)

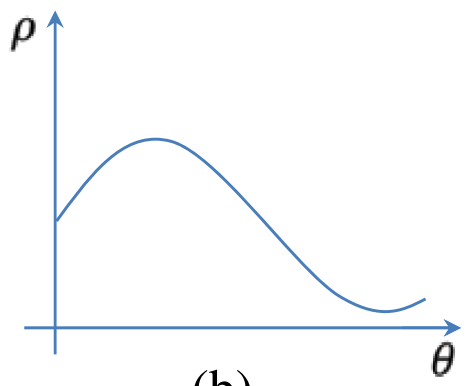

(b)

Figure 5.3: The Hough transform of a straight line. (a)Image space. (b)Parameter space.

The $\theta$ can be restricted to $[0, \pi)$, then the normal parameter for a line is 
unique. With this restriction, every point in the $\mathrm{x}-\mathrm{y}$ space can be mapped as a sinusoidal curve in the $(\theta, \rho)$ parameter space. Similarly, every straight line in the $\mathrm{x}-\mathrm{y}$ space can be mapped as a point in the $(\theta, \rho)$ parameter space.

To do the Hough transform, first we need to find out the potential edges in the image. Edges are locations in the image with strong intensity contrast. Edges often occur at image locations representing object boundaries, so edge detection is extensively used in image segmentation when we want to divide the image into areas corresponding to different objects.
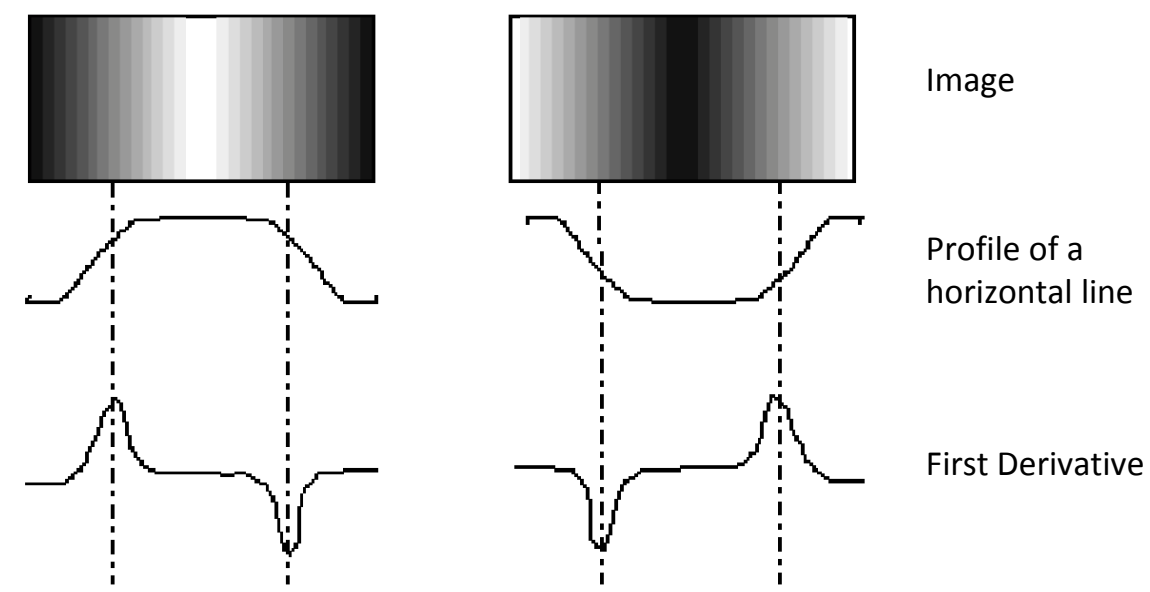

First Derivative

Figure 5.4: Gradient of the image [53].

Edges can be detected by applying a high pass frequency filter in the Fourier domain or by convolving the image with an appropriate kernel in the spatial domain. In practice, edge detection is performed in the spatial domain, because it is computationally less expensive and often yields better results. Since edges correspond to strong illumination gradients, the derivatives of the image are used for calculating the edges. After smoothing the image and eliminating the noise, the next step is to find the potential edge points by calculating the gradient of the image. Most edge detection methods work on the assumption that an edge occurs where there is a discontinuity in the intensity function or a very steep intensity gradient in the image as shown in Figure 5.4. Most edge-detecting operators can be thought of as gradient-calculators. Among them, the Sobel Kernels are based on the simple idea that the central difference between rows is the horizontal gradient and the central difference between columns is the vertical gradient, referring to Eq (5.7). Using the Sobel Kernels, the convolutions are performed on 
the image to obtain the horizontal gradient $\left(G_{x}\right)$ and vertical gradient $\left(G_{y}\right)$. The absolute gradient magnitude $G$ is calculated by the mean square root of $\left(G_{x}\right)$ and $\left(G_{y}\right)$ :

$$
\begin{gathered}
G=\sqrt{G_{x}^{2}+G_{y}^{2}} \\
S_{x}=\left(\begin{array}{ccc}
-1 & 0 & 1 \\
-2 & 0 & 2 \\
-1 & 0 & 1
\end{array}\right) \quad S_{y}=\left(\begin{array}{ccc}
1 & 2 & 1 \\
0 & 0 & 0 \\
-1 & -2 & -1
\end{array}\right)
\end{gathered}
$$

To get edges a threshold is needed which is often set as one fourth of the maximum gradient value. If the gradient of one pixel is above the threshold, it is considered as an potential edge point, and in the edge map its value is set to 1 . Otherwise if the gradient of the pixel is below the threshold, it is discarded, and in the edge map its value is set to 0 . The obtained binary edge map is the input of Hough transform.

To implement the concept of Hough transform, the parameter space is constructed by a two-dimentional array of accumulator cells as shown in Fig.5.5. The quantization is confined to the region $0 \leq \theta<\pi,-I \leq \rho \leq I$, where $\mathrm{I}$ is the possible largest $\rho$ of lines in the image. Initially, the accumulator cells are set to zero. For each potential point $\left(x_{i}, y_{i}\right)$ of the image, the corresponding sinusoidal curve is projected into the array. This is done for every potential point in the image space, we let the $\theta$ go through $[0, \pi)$, then solve the $\rho$ using Eq.(5.5). The obtained value of $\rho$ is rounded off to the nearest value in the $\rho$-axis. For angle $\theta_{i}$, if it results in solution $\rho_{j}$, We add 1 into the cell $\mathrm{AC}\left(\theta_{i}, \rho_{j}\right)$. This accumulating process is called voting. Thus, a given cell in the two-dimensional accumulating array eventually records the total number of curves passing through it. The longer a line is, the higher the count it will receive at the cell, which corresponds to its feature $(\theta$ and $\rho)$ in the accumulating array. After all of the potential points in the image are finished voting, the cells with high count will be selected. 


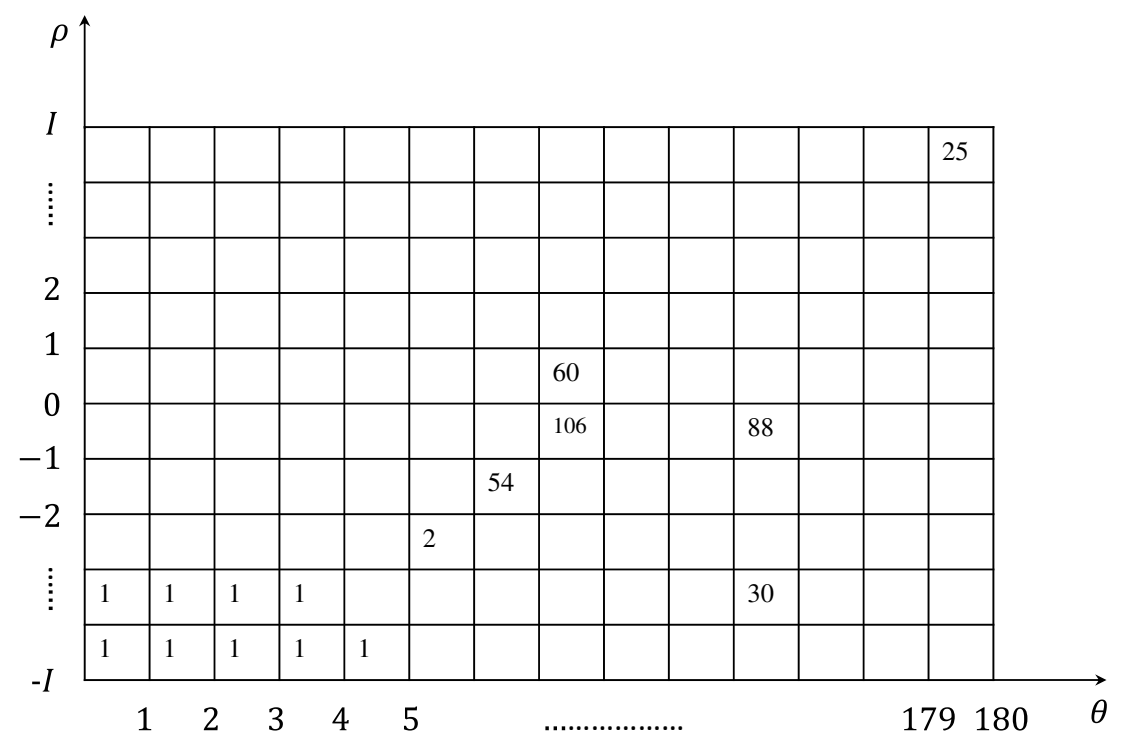

Figure 5.5: Parameter space.

\subsection{Proposed Bit-Plane Based Early-Stop Hough Transform}

The main drawbacks of the Hough transform are its high memory requirements and computational complexity, and these impose a limitation on the use of the transform for real-time applications. Various improvements have been suggested in order to make the transform computationally efficient or to facilitate its use in real time. These improvements can be divided into two broad categories. The first category is based on modifications of the algorithm to facilitate fast implementations on affordable computations, and the second category focuses on the use of dedicated hardware to accomplish the same task. In this section, we'll focus on both of these two approaches.

Most existing Hough transform computation methods are not parallelized to compute the Hough transform of several pixels simultaneously. In this chapter, an efficient local-voting early-stop Hough transform scheme and its hardware implementation is proposed based on the system-level requirement of our proposed bit-plane based zero processing time DIP system. It is shown that the proposed method yields significant performance gains. Further, optimizations for managing area and power costs are also proposed. 


\subsubsection{Overall Algorithm Description}

On the basis of the successful realization of our multi-integration low-power sensor, we defined the specification for our low-power real-time image processing system:

1. The sensor works in the 1-bit multi-integration resolution, and generates the images from the MSB plane to LSB plane.

2. Utilizing the long image integration time (which is normally longer than the image processing time), the processing circuit is set to work in this time period and also work in the 1-bit precision. The shortest precision and sufficient processing time enable us to run the processing circuit at the lowest working frequency and operating voltage.

3. The processing of any image plane must be finished within the integration period of the next image plane. Based on the processing results, decision should be made on whether the processing can stop early and finish, or needs to be continued with the information of the next image plane/planes.

4. Since the processing time is hidden in the image capture process, our processing can be addressed as "zero-time processing" as no extra processing time is needed. However, the cost of the processing circuit is an important concern. The area and power consumption of the processing circuit should be kept as low as possible.

As for the Hough transform, the bottleneck for low-cost and real-time implementation is its expensive computations and large memory size associated to the voting process. To attain our first goal of real-time processing, we need to confine the number of computations to a reasonable level. Simply put, the primary clock of the sensor is $200 \mathrm{~K}-300 \mathrm{KMHz}$. The integration of the second image plane BP6 is the shortest integration time among all. It is treated as the reference processing time. Fig.5.6 shows the timing diagram of the integration process of our sensor. It is separated by 8 phases in order to get each bit of the counter code ready ( from the Most Significant Bit (MSB) to the Least Significant Bit (LSB)). 


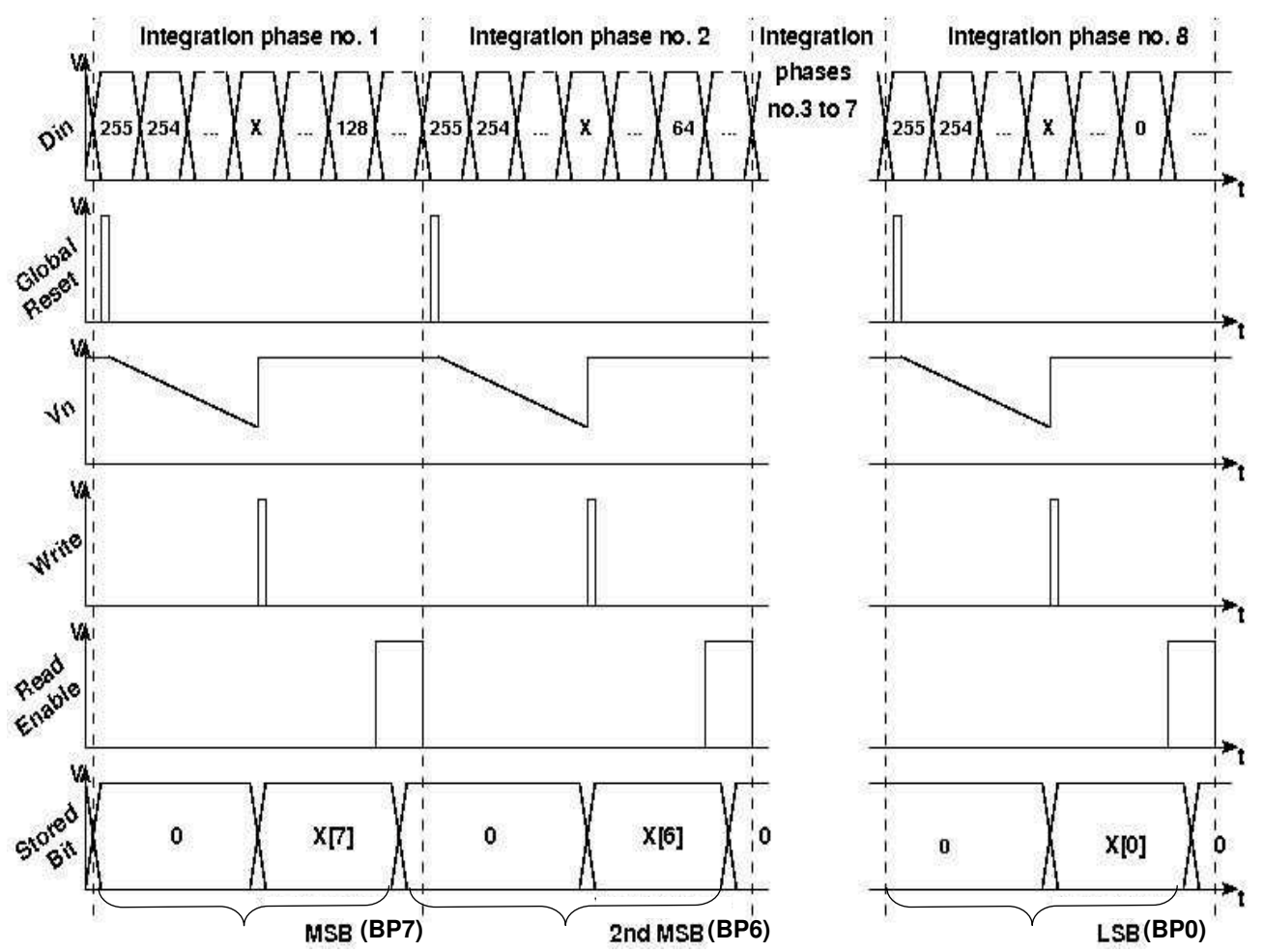

Figure 5.6: The timing diagram of the proposed multi-reset integration sensor.

Table 5.1: The clock cycle entry for different counter range.

\begin{tabular}{c|c|c|c}
\hline \hline Counter value & Clock division & Counter value & Clock division \\
\hline $255-208$ & 1 & $19-15$ & 224 \\
$207-161$ & 2 & $14-12$ & 383 \\
$160-137$ & 3 & 11 & 535 \\
$136-120$ & 4 & 10 & 648 \\
$119-109$ & 5 & 9 & 800 \\
$108-100$ & 6 & 8 & 1012 \\
$99-83$ & 8 & 7 & 1322 \\
$82-70$ & 12 & 6 & 1799 \\
$69-60$ & 16 & 5 & 2591 \\
$59-49$ & 23 & 4 & 4048 \\
$48-40$ & 33 & 3 & 7197 \\
$39-33$ & 50 & 2 & 16193 \\
$32-26$ & 77 & 1 & 64770 \\
$25-20$ & 134 & 0 & Overflow \\
\hline \hline
\end{tabular}


In each integration phase, only 1 bit code is stored. In the integration time of next bit, the previous bits can be read out and be processed at the same time. From table 5.1, we can get that there are 690 cycles in the integration phase of BP6:

Total clock cycle $=(255-208) \times 1+(207-161) \times 2+(160-137) \times 3+(136$ $-120) \times 4+(119-109) \times 5+(108-100) \times 6+(99-83) \times 8+(82-70) \times 12+(69$ $-64) \times 16=690$

Assuming we run the processing circuit at $100 \mathrm{MHz}$, it means that we can have up to 345000 cycles for the processing period. However, for a $256 \times 256$-pixel image, the voting step alone would need around $11796480(0.5 \times 256 \times 256 \times 180 \times 2)$ number of computations, which is 34 times the allowable 345000 operations. Similarly for the voting step, a $360 \mathrm{~K}$-bit $(180 \times 256 \times 8$-bit $)$ memory is needed for recording the histogram of the voting. In $0.35 \mu \mathrm{m}$ CMOS process, such a RAM can occupy more than $19 \mathrm{~mm}^{2}$ of area, which is not reasonable for our targeted low-power portable devices. Hence, our optimization will focus on how to reduce the number of computations and memory requirements, especially in the voting stage.

First let's examine an ordinary accumulating array. Fig.5.7 shows the 3-D version of the accumulating array, where the $\mathrm{x}$ axis is the $\theta$ parameter, which is quantized to 180 levels, and the y axis is the $\rho$ parameter, which is quantized to 256 levels. The $\mathrm{z}$ axis shows the intensity of the voting. The top high pillars correspond to the lines found in the image plane, the number of which depends on the number of lines contained in the image. The selected top counts are picked out and independently shown in Fig.5.8. We can see that, even in Fig.5.7, only a portion of the accumulating array is occupied. After thresholding, which is the significant step for final line detection, only a few number of cells (less than $1 \%$ ) remains meaningful, others will be discarded directly. Clearly, most of the accumulating array is wasted.

To shrink the size of the accumulating array, reducing the quantization levels of $\theta$ and $\rho$ is not a perfect solution. The detection results are sensitive to the quantization of both $\theta$ and $\rho$. Coarser quantization gives worse resolution. 


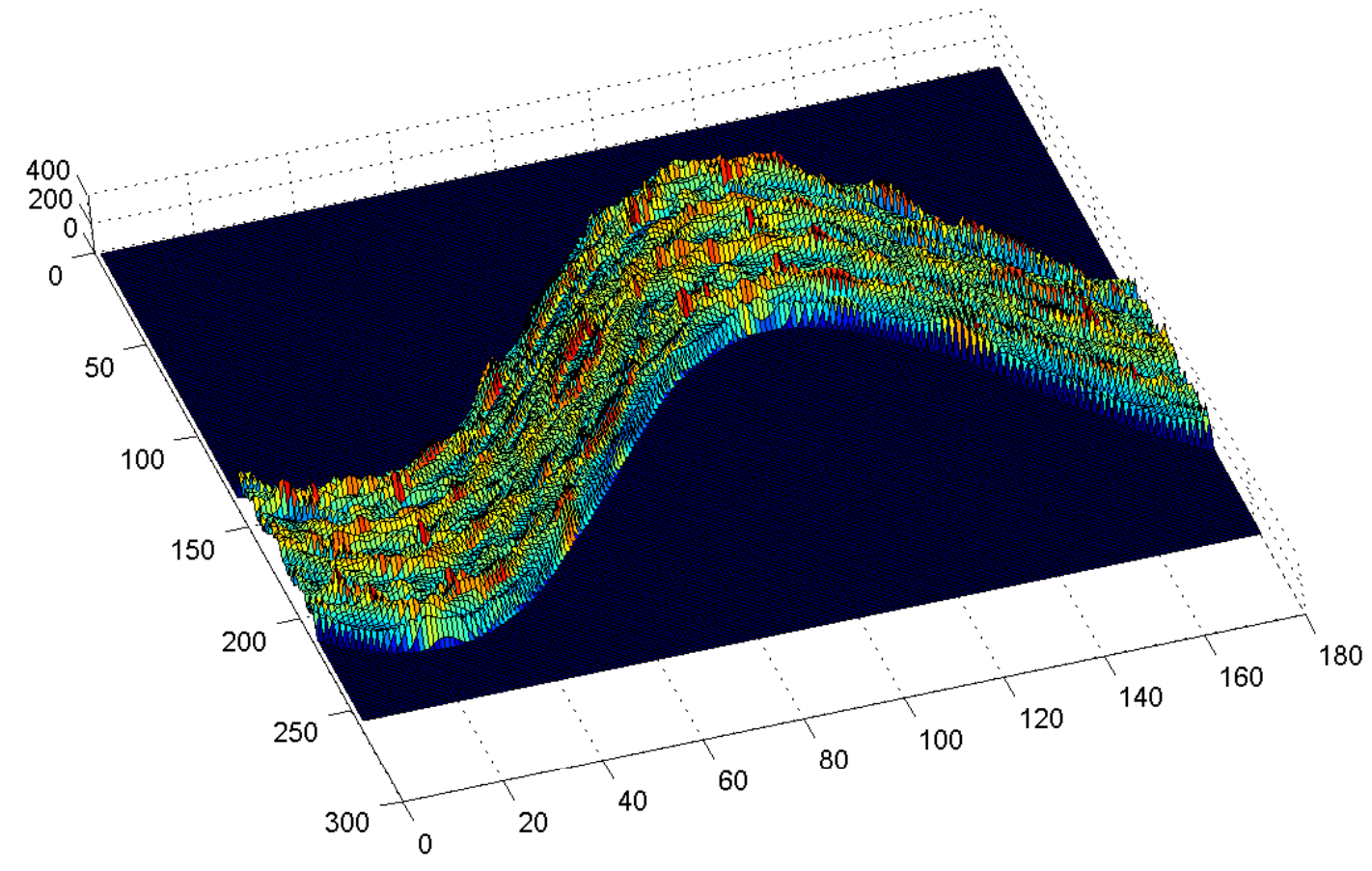

Figure 5.7: The utilization rate of the accumulating array.

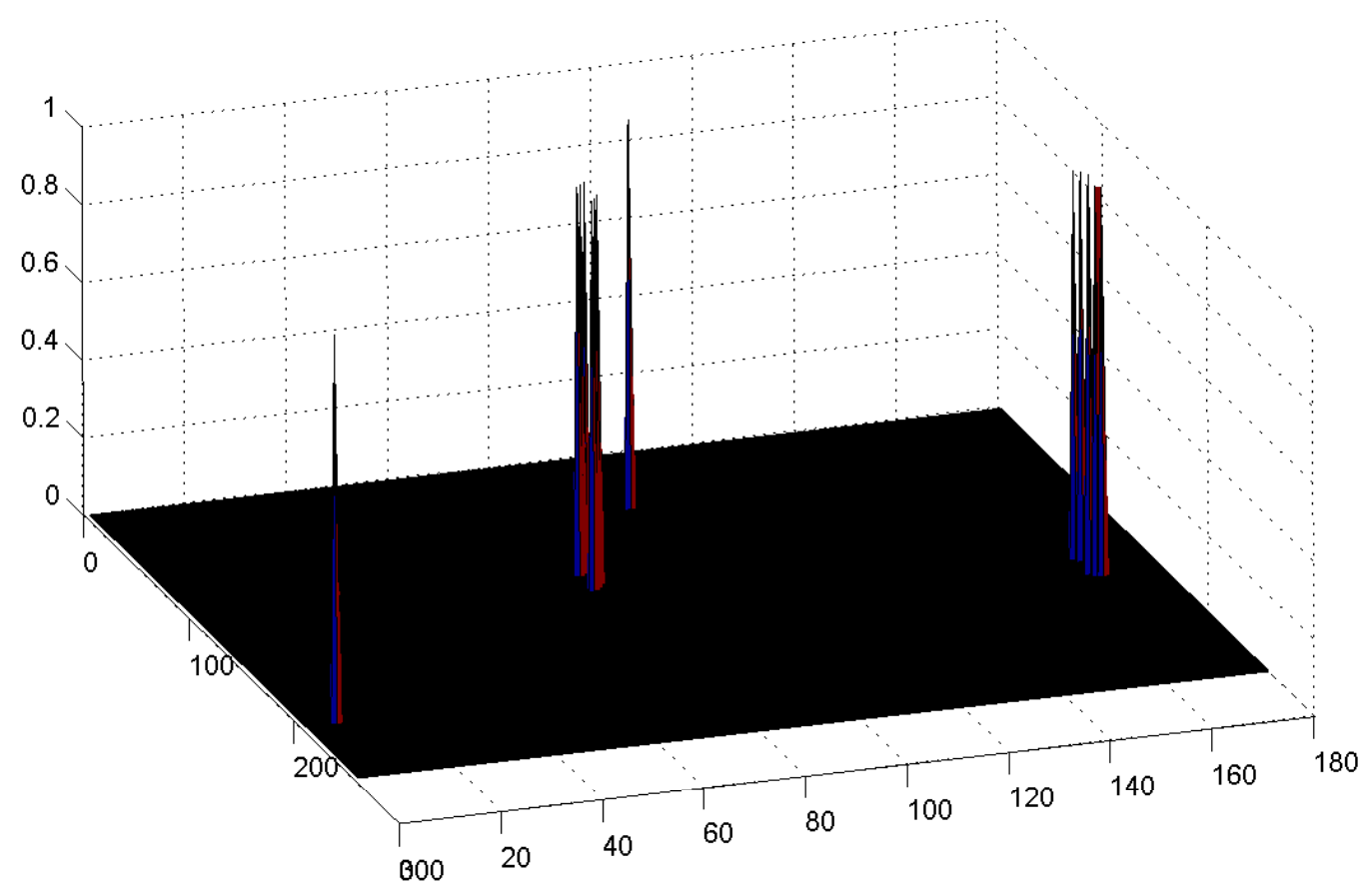

Figure 5.8: The lines found in the parameter space. 
To address this problem, our view is that, no matter how long a line is, we don't care about whether the line is detected as a whole. Instead, we only care about whether it can be found, even if it is separated in several pieces. From this point of view, to detect a line, we don't need the contribution of all pixels in the image. The meaning of doing global voting is to find the accurate length (actually the accurate number of colinear points) of each line. If this feature is not significant anymore, we can replace the global voting with local voting as long as its range is properly selected so that the lines are not be left out.

Considering both detection efficiency and memory usage, for a $256 \times 256$-pixel image, we partition it into 16 sub-images of $64 \times 64$ size. Because the threshold of the voting is often set as half of the maximum count in the accumulating array, this size implies that for a $256 \times 256$-pixel image, a line of 32 -pixel long, which is $1 / 8$ of the range of the image, can definitely be found. In this way, we can shrink the memory size of the voting stage from $256 \times 180 \times 8$-bit to $64 \times 180 \times 6$-bit, which constitutes $18.8 \%$ of the original size.

On the other hand, referring to Fig.5.1, we can see that from bit plane 7 to bit plane 0 , different levels of shape and content information are conveyed. However, the first 4 bit planes contains the most meaningful information. The last 4 bit planes constitute mainly slightest details or even details like imaging noise. On the basis of bit plane processing, we propose a new early-stop strategy, which uses as little bit planes as possible, to improve the efficiency of the processing. In view of hardware implementation, we believe that the bit plane based approach can replace all of the arithmetic operations required. In some cases, bit planes can be neglected to improve the processing efficiency, while maintaining the accuracy.

Our proposed bit-plane based early-stop local-voting Hough transform is shown in Fig.5.9.

Step 1. Gradient calculation. The input bit plane image is processed using Sobel kernels in this step, and the gradient magnitude is calculated by the square root of the horizontal and vertical gradients. 


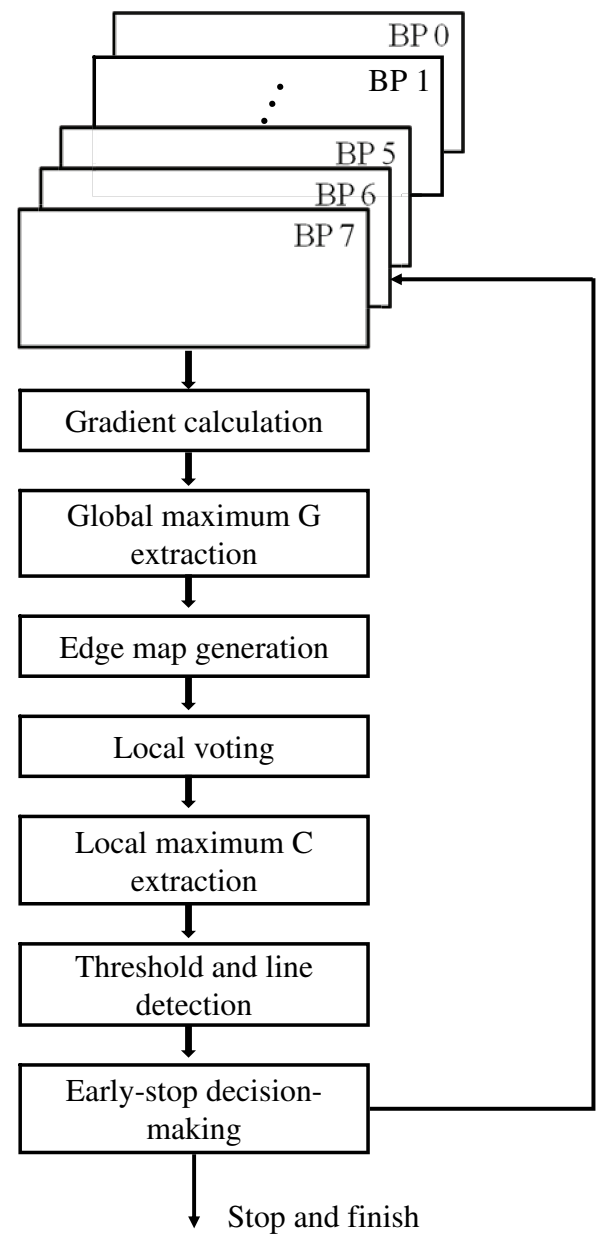

Figure 5.9: Stages of the proposed Hough algorithm. 
Step 2. Global maximum gradient extraction. To obtain the edge image, which defines the potential points lying on edges (lines), the global maximum gradient needs to be extracted and $1 / 4$ of it is set to be the threshold of the edge map generation.

Step 3. Edge map generation. For every pixel of the bit plane image, if its gradient magnitude is bigger than the threshold, this pixel is considered as a potential point, and its value is set to 1 . On the contrary, if the gradient magnitude of a pixel is smaller than the threshold, this pixel is discarded for the voting step, and its value is set to 0. After this a binary edge map is generated.

Step 4. Local voting. The edge map is divided into $4 \times 4$ non-overlapped sub-images for a fixed resolution of the angular parameter $(\theta)$ and a compressed range of the length parameter $(\rho)$. The size of the parameter space is reduced according to the diagonal of the sub-image. The bits of each accumulating cell is also reduced due to reduced number of pixels which will vote. For a $256 \times 256$ pixel image, the Hough space can be reduced to $256 \times 180 \times 8$-bit to $64 \times 180 \times 6$-bit, which constitutes $18.8 \%$ of the original size.

Step 5. Local maximum count extraction. Half of the maximum count in the accumulating array is often set as the threshold for the last step's line detection.

Step 6. Thresholding and line detection. The cells whose counts are bigger than the threshold will be considered as lines detected in the image space, and their coordinates $\theta$ and $\rho$ will be recorded and used for line back annotation.

Step 7. Early-stop decision making. Based on the line detection performance of the current bit plane, decision is made on whether to continue to process the next bit plane or early-stop at this plane. For different bit planes, we use different thresholds for line detection. Because we think points detected in BP7 have a more important probability to belong to the line than points detected on BP6 and so on. The criterion for deciding the early-stop is, after step 6 , based on the percentage of potential points, which are selected out and remain undecided, if there are more than $20 \%$ of potential points are undecided, it means that there are still many lines not be found. In this case, the next bit plane needs to be 
processed and contributes to the final result. If more than $80 \%$ of the potential points are detected as lines, it means most of the lines have been found, so the process can stop the processing here.

Fig.5.10 shows the performance of our proposed algorithm. Column (a) corresponds to the original images: ball, building, divergence, grid, sketch, and direction board; column (b) gives the results using traditional 8-bit pixel globalvoting Hough transform; column (c) corresponds to the results using bit-plane based global-voting early-stop Hough transform; and column (d) gives the results using our proposed bit-plane based local-voting early-stop Hough transform.

From the above simulation results, we can note that:

1. For most of the images, we do not need the full 8-bit pixel precision. In fig 5.10, images ball, divergence, and direction board are processed only using a single plane - their MSB planes. Images building, grid, and sketch are processed using two planes respectively: their MSB plane and second MSB plane. In general, adding more bit planes into the processing can bring in more information and produce better performance. However, there is a bound for the number of planes taking part in the processing. After the bound is exceeded, no obvious performance enhancement can be observed. In most of the cases, less than 4 bit planes are enough to extract the main shapes contained in the images. We can see that the processing results of the bit-plane scheme and early-stop scheme are comparable with traditional full-precision scheme, sometimes even better.

2. For the shape detection or image segmentation, it is not always true that the full-precision processing is superior to the bit-plane scheme and early-stop scheme because the latter would lose some information. Besides the computation reduction, the merit of the bit-plane scheme and early-stop scheme is that they can discard the details of the image and highlight the shape information. Especially for those intricate images, the disturbing details can be removed, which facilitates the following processing instead of neglecting any "entropy". For example, in the grid image, there is a weak line around the middle. In the traditional HT, when performing the edge points detection, it is neglected because its intensity is too weak. It cannot reach the threshold for being a candidate edge points. 

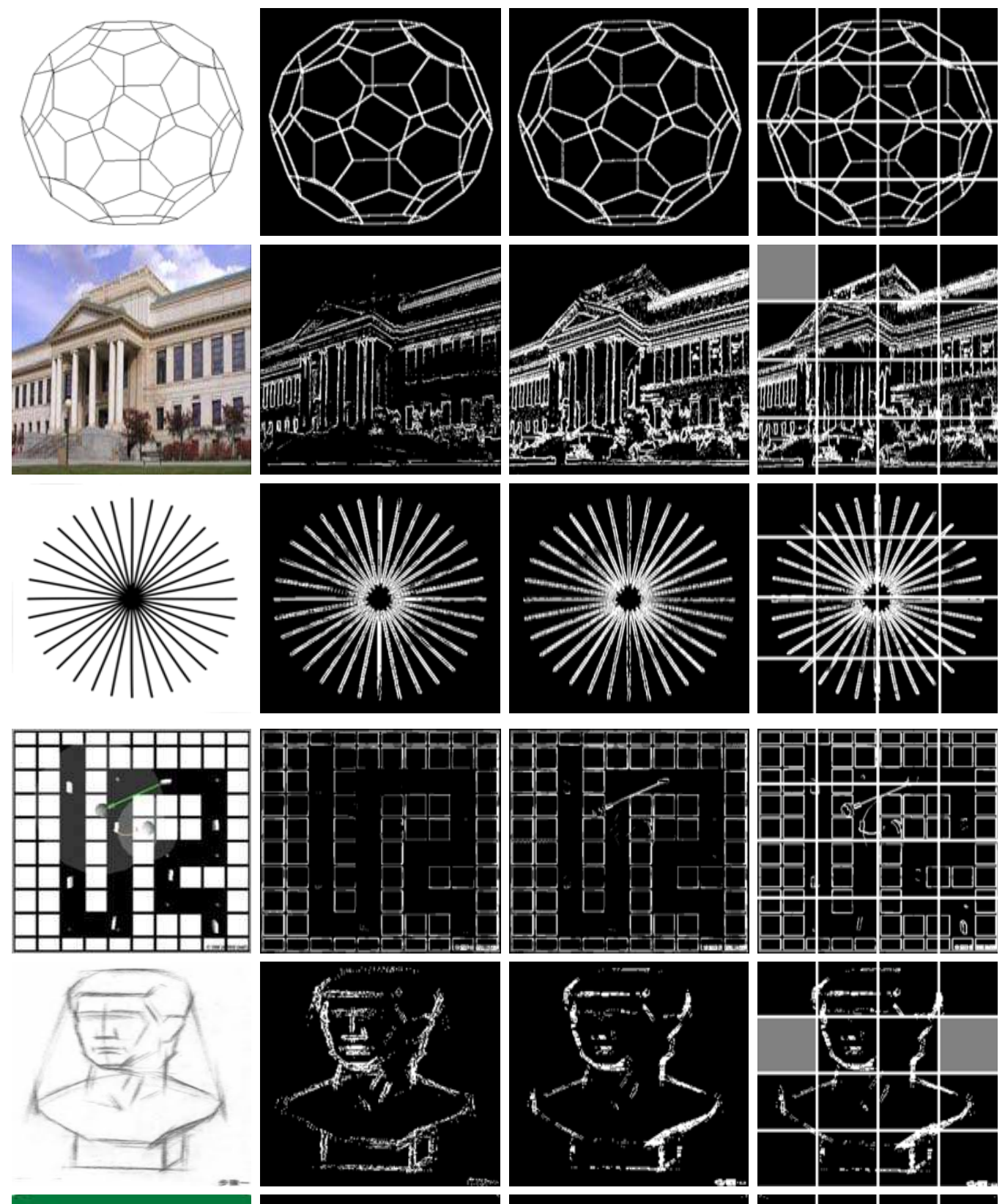

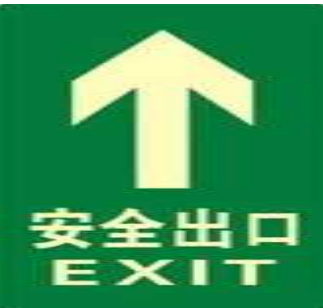

(a)

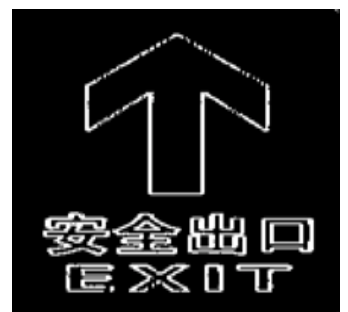

(b)

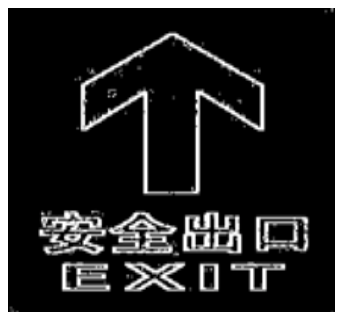

(c)

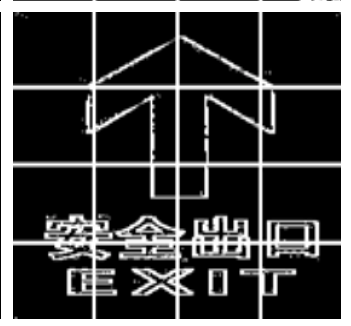

(d)

Figure 5.10: Performance comparison of different Hough transform schemes. Column(a): original images; column(b): traditional full-precision global-voting algorithm; column(c): our proposed bit-plane early-stop global-voting algorithm; column(d): our proposed bit-plane early-stop local-voting algorithm. 
But in our bit-plane approach, the intensity is compared at the same plane level. It can be well detected.

3. Besides the memory reduction, the local-voting approach happens to address another disadvantage of the global-voting. When there are many long lines in the image, the threshold for detecting lines will be raised to a high level. In such a case, the short lines will be completely shielded. Examples can be found in the fourth row of Fig.5.10. In image grid, most of the lines are long lines, which span the whole image. The short white stripes, which appear on the black background are completely shielded, referring to row 4, column (b) and (c). In the local-voting scheme, this problem is perfectly addressed because the threshold of the sub-image is reduced to at least $1 / 4$ of the global one, and every sub-image has its own threshold to cater for their own situations. Using the local-voting strategy, all of the short lines are well detected.

\subsubsection{Hardware Optimization and Implementation}

As mentioned in the previous chapter, local-voting scheme is utilized to reduce the memory size and to address the short line detection problem. In our hardware implementation, the images of $128 \times 128$ pixels are used. The images are partitioned into 4 non-overlapped $64 \times 64$-pixel sub-images and they are processed serially to utilize the hardware resources efficiently. In Fig.5.11, a block diagram of the implemented bit-plane local-voting early-stop line detection algorithm is shown.

First the Sobel kernels are applied on the bit plane image to extract the potential edge points. In the gradient convolution step, the previous row/column and the next row/column are required to be involved in the computation of the current row/column. Hence, 3 on-chip row/column buffers are needed to store the three rows/columns. In our architecture, the source image data is stored in a memory and more than one pixel can be provided during each cycle - the complete row (64-bit) of the sub-images can be read/wrote during each cycle. Therefore, in each clock cycle, we read 3 complete rows from one 

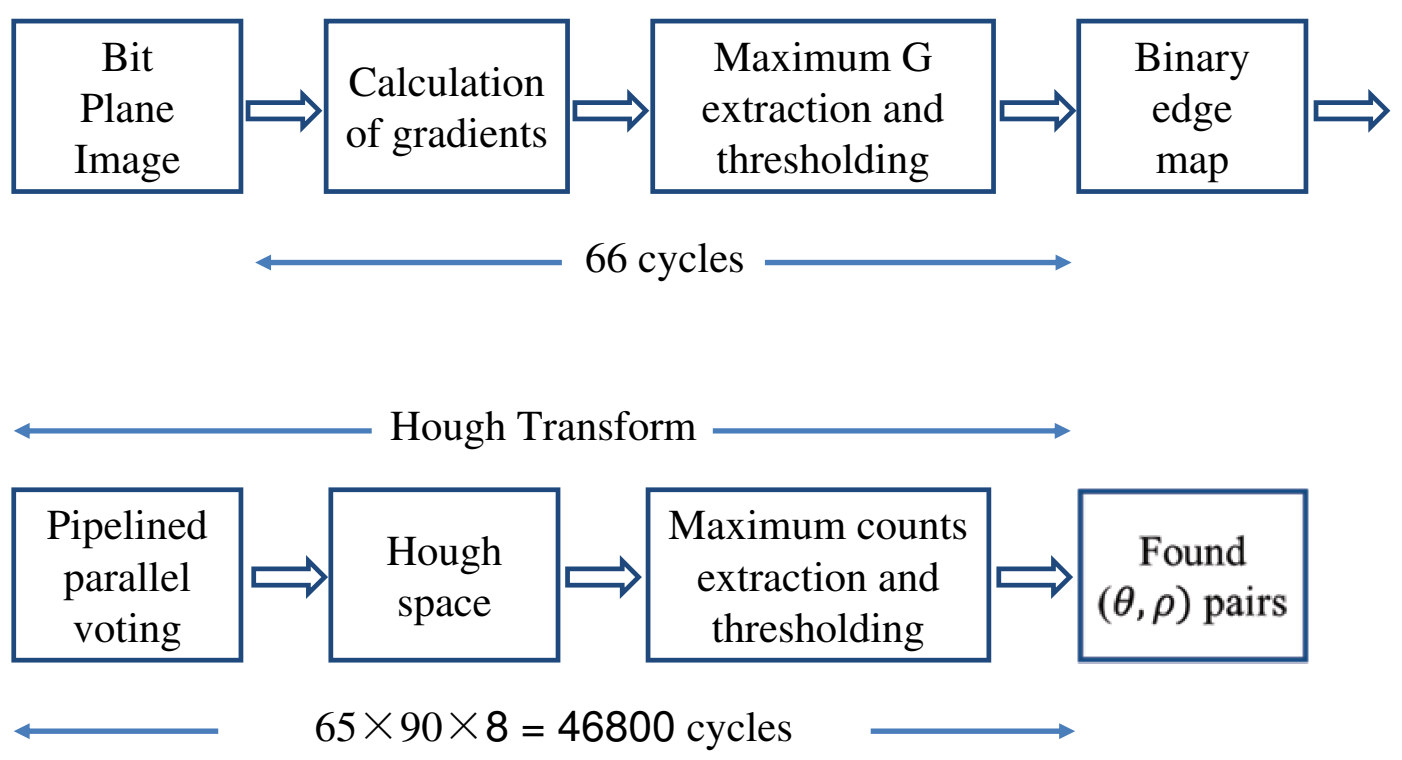

Figure 5.11: Block diagram of the Hough transform hardware implementation.

of the sub-images, and put them into three 64-bit buffers: reg_precious_line, reg_current_line, and reg_next_line. In the first cycle, row 0, row 1, and row 2 are loaded into reg_precious_line, reg_current_line, and reg_next_line respectively. In the second cycle, row 1 , row 2 , and row 3 need to be loaded into reg_precious_line, reg_current_line, and reg_next_line respectively. We can see that reg_precious_line and reg_current_line need to always load data from reg_current_line and reg_next_line cycle by cycle, as show in Fig.5.12(a).

To avoid such frequent and wasteful data transferring, we use a simple control to switch the roles of the 3 row buffers. Referring to Fig.5.12(b), in the first clock cycle, state 1 is enabled to load row 0, row 1, and row 2 into reg_precious_line, reg_current_line, and reg_next_line respectively. State 2 and state 3 are then used in order to turn the "the current row" of last step to "the previous row" in the current step, "the next row" of last step to "the current row" in the current step, and the new loaded row as "the next row" in the current step. The 3 states are used in turn to make the right sequence of the rows loaded into the 3 row buffers. As a result, unnecessary data exchange among the buffers are removed.

With the aid of the row buffers, we could obtain all the involved data for both horizontal and vertical gradient computations. Parallel horizontal and vertical 


$$
\text { Sub-image }(64 \times 64) \quad \text { Cycle } 1 \quad \text { Cycle } 2 \quad \text { Cycle } 3
$$

\begin{tabular}{|c|c|c|c|}
\hline Row 0 & reg_previous_line & & \\
\hline Row 1 & reg_current_line & reg_previous_line & \\
\hline Row 2 & reg_next_line & reg_current_line & reg_previous_line \\
\hline Row 3 & & reg_next_line & reg_current_line \\
\hline Row 4 & & & reg_next_line \\
\hline
\end{tabular}

(a)

$\underline{\text { State } 1}$

Buffer $\begin{gathered}\text { Gradient } \\ \text { computation module }\end{gathered}$
State 2

Gradient computation module

\section{Buffer}

\begin{tabular}{|l|l|}
\hline row $i-1$ \\
\hline row $i$ \\
\hline row $i+1$ \\
\hline previous row \\
\hline next row row \\
\hline
\end{tabular}

(b)
State 3

Gradient Buffer computation module

\begin{tabular}{|l|l|l|}
\hline row $i-1$ & $\longrightarrow$ & previous row \\
\hline row $i$ & $\longrightarrow$ & current row \\
\hline row $i+1$ & $\longrightarrow$ & next row \\
\hline
\end{tabular}

\begin{tabular}{|l|l|}
\hline row $i-1$ \\
\hline row $i$ \\
\hline row $i+1$ \\
\hline previous row \\
\hline current row \\
\hline next row \\
\hline
\end{tabular}

Figure 5.12: The row buffer scheme for the parallel gradient computation.

(a)The data sequence of the gradient computation. (b)The proposed buffer switching scheme for power reduction. 
gradient computations for the whole row is performed in our implementation. For the higher bit planes, the thresholding step becomes easier due to bit plane operation. For the edge map generation, the threshold for the gradient map is often set as one fourth of the maximum gradient. Considering the high weights of the pixels in the higher bit planes, eg. bit plane 7, as long as one pixel's gradient in bit plane 7 is larger than 0 , its full-precision gradient must be larger than one fourth of the maximum full-precision gradient of the whole image. For the higher bit plane images, the maximum gradient extraction step and the extra thresholding step can always be omitted to save power.

After the binary edge map is obtained, the voting in the Hough space begins. First the $\rho$ and $\theta$ axises of the accumulating array are quantized into 64 and 90 levels respectively for hardware efficiency. For the size of the voting cells, the counts in the cells represent the numbers of points lying on each straight lines. For the $64 \times 64$-pixel sub-image, the maximum value of each cell is smaller than 64, which means a 6-bit cell. However, the threshold for Hough transform is often set as one half of the maximum count. The largest possible threshold is 31. We do not care about the accurate values of the cells. Rather, we only care about whether the counts are larger or smaller than the threshold. Hence, we can make the cells to be 5-bit, which can represent a maximum value of 31 . Once its maximum value is achieved, it means this cell definitely corresponds to a line, and the continuous increment is meaningless. For this case, the value of the count should be kept until the end of the process. Note that not every subimage can achieve the largest possible threshold. When the precision of the cells is constrained to 5-bit, we may lose the record of the real largest count, which is responsible for generating the detection threshold. A 6-bit maximum counts recorder are needed to record the maximum count of each voting step. The half of its final value will be set as the threshold for the line detection.

The standard Hough transform implements the parameter space as an accumulating array, where each quantized cell in the array corresponds to a straight line in the image. Each cell in the array is initialized to 0. For each edge point, the corresponding sinusoidal curve is traced within the accumulating array, in- 
crementing each cell that is visited. In effect, each edge point "votes" for all lines that pass through it. After all edge points have been processed in this manner, the array is examined. The large values own higher potential to be lines that may be present in the image. When detecting whether lines are present in an image, only boundaries (or called edge points) are of interest. For the pixels whose value is 0 in the edge map, they cannot take part in the voting.

For each of the potential edge points in the edge map, 90 computations of Eq.(5.2) need to be performed because the whole range of the $\theta$ axis needs to be passed through. As explained before, the integration time for bit plane 6 is $3450 \mu \mathrm{s}$, which is the shortest integration period among all of the 8 bit planes and bit plane 7 needs to be finished within this time period. If we run the processing circuit at $100 \mathrm{MHz}$, only 345000 cycles are available for all of the processing. For a $128 \times 128$ pixels image, $A \times 64 \times 64 \times 90 \times 4=A \times 1474560$ cycles are needed for serial processing. The term $\mathrm{A}$ is the potential edge point ratio, which is $0.02-0.5$ in general. To meet the tight timing constraint, parallel hardware structure is required.

Fig.5.13 shows the parallel hardware architecture of our Hough transform algorithm. In each sub-image, there are 64 rows, every 8 rows are made as a group and processed in parallel. As shown in Fig.5.13, for the 1st row of each group, it takes 64 cycles for the 64 pixels to finish the computations for the same $\theta$ parameter. The computation results need to be written back to the accumulating array, so the rest of the rows cannot access the same cells (for same $\theta$, it's the same column) as the first row. Therefore, 8 rows are arranged to compute 8 different $\theta$ parameters in parallel, and different columns of the accumulating array are updated at the same time.

For the Hough array accumulation, in every cycle, the whole column corresponding to a certain $\theta$ needs to be read out because we don't know which cell in the column will be incremented. In one hand, to update the cells once, it takes 3 cycles: read, increment, and write back. On the other hand, parallel processing makes that every cycle 8 columns need to be read and written back. To save power and time, we changed the operation flow. By introducing a column buffer, for 


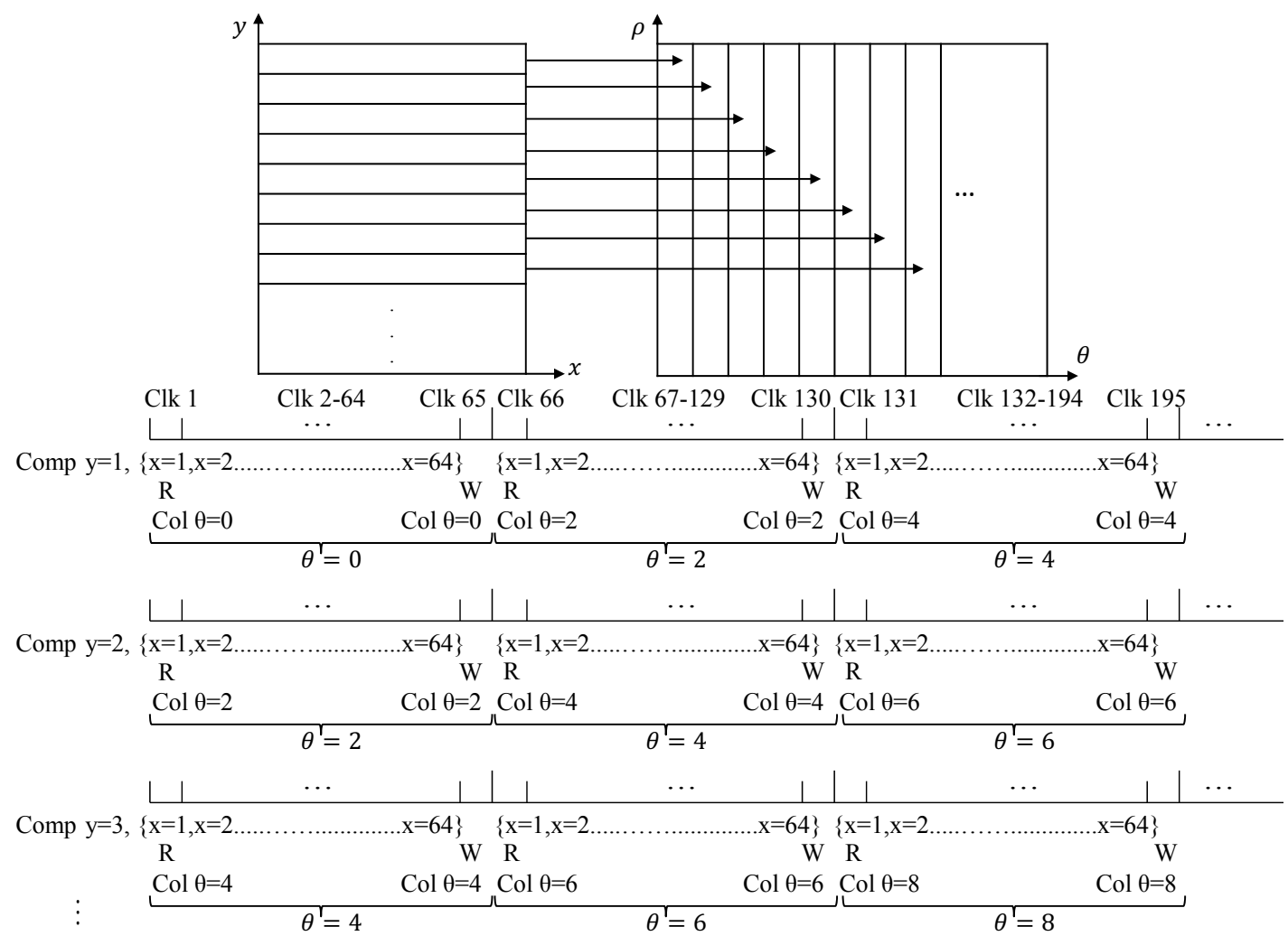

Figure 5.13: The parallel hardware architecture of our Hough transform algorithm.

each row, we read the required column just once and put it in the column buffer. In the following 64 cycles, we update the counts in the column buffer instead of the accumulating array. After the 64 cycles, the whole column buffer is used to update the corresponding column of the accumulating array. For the entire row, 65 cycles are required for the accumulating array read, $\rho$ computation, and accumulating array update for one $\theta$ value. For the whole sub-image, $65 \times 90 \times 8=46800$ cycles are required to finish all of the voting. The serial processing of the whole $128 \times 128$ pixels image needs $(66+65 \times 90 \times 8) \times 4=187464$ cycles.

For the computation of the $\rho$ parameter, for the same row, every pixel's $y$ value is the same. For the same $\theta$ phase, every pixel's $\sin \theta$ and $\cos \theta$ values are the same. For every $\theta$ phase of each row, the $y \sin \theta$ value just needs one time of computation. The only difference is $x \cos \theta$. $X^{\prime}$ value can only be integer from 1 to 64 , so multiplications for calculating $x \cos \theta$ can be replaced by the shift and addition/subtraction operations. For example, when $x=2, x \cos \theta$ can be obtained by shifting the $\cos \theta$ value read from the sincos look-up-tables by 1 bit to the left. 
When $x=47, x \cos \theta$ can be obtained by shifting the $\cos \theta$ value by 5 bits and 4 bits respectively to get $32 \cos \theta$ and $16 \cos \theta$, then decrementing the sum of these two values. Among the values of $32 \cos \theta, 16 \cos \theta, 8 \cos \theta, 4 \cos \theta$, and $2 \cos \theta$ got from the simple shift operations, at most 4 levels of addition/subtraction are enough for computing every possible $x$.

\subsubsection{Experimental Results and Analysis}

The entire design was simulated and implemented using the software package ISE 9.1 from Xilinx and tested on a Virtex-4 XC4VFX100 development board. The processed images were captured by the image sensors developed in our S2IS lab. $128 \times 128$-pixel images were used. The test images were first read and stored in the RAM of the FPGA. After the processing, the $(\theta, \rho)$ pairs can be obtained to represent the position of the lines contained in the image. Running at $100 \mathrm{MHz}$ clock frequency, the whole processing employs 187464 cycles to obtain the $\theta$ and $\rho$ parameters of the lines contained in the image. The choice of using the FPGA platform is due to our design is a tentative prototype for validating the proposed bit-plane based zero-time processing scheme. In the future work, this module will be expanded for more geometry shapes detection, and the ASIC platform will be chosen for the implementation of the integrated DIP system (including the image sensor and the processing circuits).

Regarding the utilization of resources of the FPGA, Table 5.2 shows the report of the synthesis summary of Xilinx. We can observe that with the adopted schemes, the design is solved loosely with a total equivalent gate count of 0.4 million gates (414,490 gates). For the processing of the MSB plane, we can reduce the operating frequency to $55 \mathrm{MHz}$. For the second MSB plane - bit plane 6 , $8125 \mu \mathrm{s}$ can be allocated for the processing because of the extension of integration period of bit plane 5 . In this stage, $25 \mathrm{MHz}$ clock frequency is enough for the processing. For later bit planes, the clock frequency can be reduced even lower to save power.

Table 5.3 shows the performance comparison between our work and related 
Table 5.2: The summary of the logic utilization of the FPGA device.

\begin{tabular}{|l|c|c|c|}
\hline \hline Resource & Used & Available & Utilization rate \\
\hline Number of occupied Slices & 23,414 & 42,176 & $55 \%$ \\
Number of Slice Flip Flops & 1,388 & 84,352 & $1 \%$ \\
Number of 4 input LUTs & 31,662 & 84,352 & $38 \%$ \\
Number of bonded IOBs & 27 & 576 & $5 \%$ \\
Number of DSP48s & 4 & 160 & $2 \%$ \\
Number of Block RAMs & $50 \mathrm{~K}$ & $6768 \mathrm{~K}$ & $1 \%$ \\
\hline \hline
\end{tabular}

works. Different parameters are normalized to make them easy to be compared. Processing the same size of image, our algorithm needs the least number of transistors. We use an efficiency feature, which represents how many pixels can be processed using every 10000 gates every MHz. Our work shows a much larger efficiency compared with prior works. Heuristically speaking, combining with the power management technique, the higher efficiency architecture would obtain larger power savings than the low efficiency architecture.

Table 5.3: Performance Comparison with Related Works

\begin{tabular}{|c|c|c|c|c|c|}
\hline & $\begin{array}{l}\text { Image Size } \\
(\mathrm{M} \times \mathrm{N})\end{array}$ & $\begin{array}{c}\text { Technology } \\
\text { (FPGA/ASIC) }\end{array}$ & $\begin{array}{l}\text { Clock } \\
(\mathrm{MHz})\end{array}$ & $\begin{array}{c}\text { Area } \\
\text { (Equivalent gates) }\end{array}$ & $\begin{array}{c}\text { Efficiency } \\
\text { (pixels/ } \\
10000 \text { gates*1MHz) }\end{array}$ \\
\hline Our work & $\begin{array}{c}128 \times 128 \\
(1.00) \\
\end{array}$ & FPGA & $\begin{array}{l}55 \mathrm{MHz}(\mathrm{BP} 7) \\
25 \mathrm{MHz}(\mathrm{BP} 6)\end{array}$ & $\begin{array}{c}414,490 \\
(1.00) \\
\end{array}$ & $\begin{array}{c}7.19(\mathrm{BP} 7) \\
15.82(\mathrm{BP} 6) \\
\end{array}$ \\
\hline$\overline{[54]}$ & $\begin{array}{c}176 \times 120 \\
(1.29)\end{array}$ & FPGA & - & $\begin{array}{c}4,138,560 \\
(9.98)\end{array}$ & $1.02^{*}$ \\
\hline$[55]$ & $\begin{array}{c}44 \times 36 \\
(0.10)\end{array}$ & FPGA & $70 \mathrm{MHz}$ & $\begin{array}{c}1,000,000 \\
(2.41)\end{array}$ & 0.23 \\
\hline$[56]$ & $\begin{array}{c}400 \times 500 \\
(12.20)\end{array}$ & $\begin{array}{c}\text { ASIC } \\
\text { (AMS 0.6um) }\end{array}$ & $100 \mathrm{MHz}$ & $\begin{array}{c}50 \mathrm{~mm} 2 \\
\left(12.01^{* *}\right)\end{array}$ & 4.02 \\
\hline
\end{tabular}

* estimated using $50 \mathrm{MHz}$.

** not including the edge points detection module.

Fig 5.14 shows the experimental results of our proposed algorithm. The test images are of $128 \times 128$-pixel size, and only their significant plane (BP7) is used for processing. Running at $55 \mathrm{MHz}$, the test images can be processed within the integration time of BP6 (around 3.45ms). From a system point of view, zerotime processing is achieved. Besides, the processing is achieved by scanning rows instead of edge pixels, so no matter how many feature points there are, constant 
processing time is achieved. This would benefit the following DVFS. The results obtained demonstrate two potential applications for our algorithm including traffic signs detection and handwritten alphanumeric characters recognition.

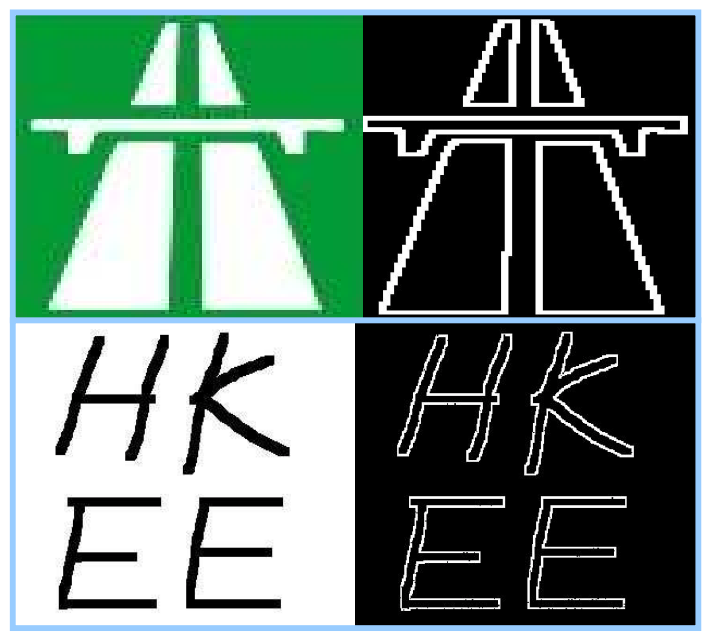

Figure 5.14: Experimental results of proposed algorithm. (a)Traffic Signs Detection. (b)Handwritten Alphanumeric Characters Recognition.

\subsection{Summary}

In this chapter, a bit-plane based zero-time line detection DIP module is proposed. The bit-plane scheme makes part of the arithmetic operations be realized by only binary Boolean functions. The local-voting scheme helped to reduce the memory to $16 \%$ of the original size. The computation complexity for voting is also reduced due to the reduced size of the voting array. The early-stop scheme enables the use of a minimum number of bit planes to greatly reduce the image capture and processing time. The hardware implementations and optimizations were realized on a Xilinx Virtex-4 FPGA. The experimental results demonstrate that our proposed algorithm improves processing efficiency, and greatly reduces computation load and power consumption, while maintaining the accuracy. 


\section{Chapter 6}

\section{Conclusion and Future Work}

To enable the design of more energy efficient DIP cores, this thesis has first investigated the optimal operating point (operating precision, supply voltage, clock frequency) of the most important arithmetic block, that is the multiplier. Our work has led to the design, implementation and fabrication of a multi-precision reconfigurable multiplier which incorporates variable precisions, parallel processing, razor-based dynamic voltage scaling (DVS), and dedicated multi-precision operands scheduling.

All of the building blocks of our reconfigurable multiplier can either work as independent smaller precision multipliers or work in parallel to perform higher precision multiplications. According to user's arbitrary requirements (eg. throughput), the dynamic voltage and frequency scaling management unit first configures the multiplier at the proper operating precision and frequency. Adapting to the run-time workload of the targeted application, razor flip-flops and the dithering voltage unit then help the voltage and frequency scaling management unit to autonomously configure the multiplier to achieve the lowest power consumption. Single-switch dithering voltage unit and razor flip-flops help to reduce the traditionally needed DVS overhead and voltage safety margins to the lowest level. Most notably, the proposed implementation does not result in silicon area or power overheads which are typically associated to reconfigurability.

To accommodate our multi-precision multiplier to various general-purpose 
mixed-precision applications, we designed an operands scheduler that can rearrange the input data and determine the optimum voltage and frequency pairs for overall minimum power consumption. Equipped with the operands scheduler, given any mixed-precision data stream and specified throughput requirement, our multi-precision DVS multiplier system can find the best combinations of processing precisions, supply voltages and operating frequencies during the execution time, such that the throughput requirement is satisfied, and the total energy is minimized.

The proposed low-power multi-precision multiplier was fabricated in AMIS $0.35 \mu \mathrm{m}$ technology. This work successfully demonstrates that multi-precision architecture can reap the benefits from the dynamic voltage scaling technique more effectively. Experimental results show that compared with the conventional fixed-width multiplier, the proposed design features $28.2 \%$ and $15.8 \%$ reduction in circuit area and power consumption respectively. With the multi-precision, voltage and frequency scaling, and parallel processing techniques applied, average power saving of $65.1 \%$ and $86.7 \%$ could be obtained. Augmented with the operands scheduler, up to $46.2 \%$ further power saving is achieved.

After the general guidelines for low power DIP design - finding out the optimal operating point is generalized, we have presented a complete system comprising a digital image sensor and processing circuitry. In digital pixel sensors (DPSs), memory elements typically occupy large silicon area, which significantly affects the sensor's size, power and cost. To enable low-power DIP systems, we proposed a novel multi-reset integration scheme, which uses smaller-precision in-pixel memory. The proposed sensor scheme can generate bit planes four by four, or just one by one. In the one by one bit plane generation mode, the long integration time of each image plane can be exploited for the processing of the previous bit plane. With the single-bit precision feature, the processing circuit can run at very low operating frequency and supply voltage, and realize zero-time ultra-low-power image processing.

Hough transform-based image segmentation was chosen for our first DIP core implementation. Traditional Hough transform has the main drawbacks of large 
computational complexity and high memory requirements, which impose a limitation on its use for real-time applications. In this work, a bit-plane based early-stop local-voting Hough transform DIP architecture was proposed and implemented. Experimental results demonstrate that our proposed algorithm greatly reduces the computation load, improves the processing efficiency, reduces the memory requirements while maintaining accuracy.

In the following, we propose future works to further extend the scope of this research:

1. Expanding the Hough transform for detecting more parametric shapes, including circles, ellipses, polygons, and corners. This would provide a more powerful image segmentation core. Different shapes need different parametric computations. A common hardware logic would need to be developed to accommodate these different computations and achieve a high re-utilization rate of hardware resources.

2. An interesting idea for our proposed line detection algorithm is that, it can be naturally extended to do motion detection. In the motion detection task, the two parameters that need to be determined are the moving angle and displacement of the motion. These two parameters are just the processing results of our Hough transform, requiring no other further computations. To extend our algorithm to motion estimation algorithm, two successive image frames, can be compared so that motion vectors can easily be deduced.

3. An optimization to our single-switch dithering scheme (Chapter 2) could be made to improve the rise time and reduce supply voltage fluctuations. Such an optimization could further reduce the power overhead associated to oscillations of the dithering unit. It can also benefit the operands scheduler unit, as smaller input data buffer stacks could be used, with the supply voltage transitioning faster between the different levels. 


\section{Bibliography}

[1] El Gamal A., "Trends in CMOS Image Sensor Technology and Design," International Electron Devices Meeting Digest of Technical Papers, pp. 805-08, December 2002.

[2] El Gamal A. et al, "CMOS Image Sensors," IEEE Circuits and Devices Magazine, vol. 21, Issue 3, May-June 2005.

[3] Cave D.R., "Technology Insight: current status of video capsule endoscopy," Nature Clinical Practice Gastroenterology and Hepatology, no.3, pp. 158-164, 2006.

[4] Kleinfelder S. et al, "A 10,000 Frames/s CMOS Digital Pixel Sensor," IEEE Journal of Solid State Circuits, vol. 36, no. 12, pp. 2049-59, December 2001.

[5] V. Brajovic, "Computational Sensor for Global Operations in Vision," Ph.D. Thesis, The Robotics Institute, Carnegie Mellon University, January 1996.

[6] R. W. Brodersen, A. Chandrakasan, and S. Sheng, Technologies for personal communications, 1991 Symp. VISI Circ., Tokyo, Japan, pp.5-9, 1991.

[7] Weste, H. E.; Eshraghian,K.: Principles ofCMOSVLSI Design. Reading,MA,AddisonC Wesley, 1994.

[8] Holt, J.L., Hwang, J.N., "Finite precision error analysis of neural network hardware implementations" Computers, IEEE Transactions, Volume 42, Issue 3, Page(s):281 - 290, March 1993. 
[9] A. P. Chandrakdsan, S. Sheng, R. W. Brodersen, "Low-power CMOS digital design" IEEE Journal of Solid-State Circuirs,, Volume 27, Page(s):473 - 483, April 1992.

[10] Yamanaka, T.; Moshnyaga, V.G.; "Reducing multiplier energy by datadriven voltage variation" Circuits and Systems, 2004. ISCAS'04. Proceedings of the 2004 International Symposium, Volume: 2, Page(s):II - 285-8, 2004.

[11] Wei Ling; Savaria, Y.; "Variable-precision multiplier for equalizer with adaptive modulation" Circuits and Systems, 2004. MWSCAS '04. The 2004 47th Midwest Symposium, Volume: 1, Page(s):I - 553-6, 2004.

[12] Min, R.; Bhardwaj, M.; Seong-Hwan Cho; Ickes, N.; Shih, E.; Sinha, A.; Alice Wang; Chandrakasan, A.; "Energy-centric enabling tecumologies for wireless sensor networks" IEEE Wireless Communications, Volume 9,Issue: 4, Page(s): 28 - 39, 2002.

[13] Bhardwaj,M.; Min, R.; Chandrakasan, A.P.; "Quantifying and enhancing power awareness of VLSI systems" Very Large Scale Integration (VLSI) Systems, IEEE Transactions, Volume 9, Issue: 6, Page(s): 757 - 772, 2001.

[14] Wang, A.; Chandrakasan, A.P.; "Energy-aware architectures for a RealValued FFT implementation" Low Power Electronics and Design, 2003. ISLPED '03. Proceedings of the 2003 International Symposium, Page(s): 360 $-365,2003$.

[15] Hanho Lee; "A power-aware scalable pipelined Booth multiplier" SOC Conference, 2004. Proceedings. IEEE International, Page(s): 123 - 126, January 2004 .

[16] Bermak, A.; Martinez, D.; Noullet, J.-L.; "High-density 16/8/4-bit configurable multiplier" Circuits, Devices and Systems, IEE Proceedings, Volume: 144 , Page(s): 272 - 276, 1997.

[17] O. A. Pfander, R. Hacker, and H.-J. Pfleiderer; "A multiplexer-based concept for reconfigurable multiplier arrays" IEEE FPL 2004, Page(s):938 - 942, August, 2004. 
[18] Burd,T.D.; Pering, T.A.; Stratakos, A.J.; Brodersen, R.W.; "A dynamic voltage scaled microprocessor system" IEEE Journal of Solid-State Circuirs,, Volume 35, No. 11, Page(s): 1571 - 1580, November 2000.

[19] Kuroda, T.; Suzuki, K.; Mita, S.; Fujita, T.; Yamane, F.; Sano, F.; Chiba, A.; Watanabe, Y.; Matsuda, K.; Maeda, T.; Sakurai, T.; Furuyama, T.; "Variable supply-voltage scheme for low-power high-speed CMOS digital design" IEEE Journal of Solid-State Circuirs,, Volume 33, Issue: 3, Page(s): 454 - 462, March 1998.

[20] Nakai, M.; Akui, S.; Seno, K.; Meguro, T.; Seki, T.; Kondo, T.; Hashiguchi, A.; Kawahara, H.; Kumano, K.; Shimura, M.; "Dynamic voltage and frequency management for a low-power embedded microprocessor" IEEE Journal of Solid-State Circuirs,, Volume 40, Issue: 1, Page(s): 28 - 35, January 2005.

[21] Ernst, D.; Nam Sung Kim; Das, S.; Pant, S.; Rao, R.; Toan Pham; Ziesler, C.; Blaauw, D.; Austin, T.; Flautner, K.; Mudge, T.; "Razor: A Low-Power Pipeline Based on Circuit-Level Timing Speculation" Microarchitecture, 2003. MICRO-36. Proceedings. 36th Annual IEEE/ACM International Symposium on 2003, Page(s):7 - 18, 2003.

[22] Das, S.; Roberts, D.; Seokwoo Lee; Pant, S.; Blaauw, D.; Austin, T.; Flautner, K.; Mudge, T.; " A self-tuning DVS processor using delay-error detection and correction" Solid-State Circuits, IEEE Journal of Volume: 41, Issue: 4, Page(s):792 - 804, 2006.

[23] Das, S.; Tokunaga, C.; Pant, S.; Ma, W.-H.; Kalaiselvan, S.; Lai, K.; Bull, D.M.; Blaauw, D.T.; "RazorII: In Situ Error Detection and Correction for PVT and SER Tolerance" Solid-State Circuits, IEEE Journal of Volume: 44 , Issue: 1, Page(s):32 - 48, 2009.

[24] Shaolei Quan; Qiang Qiang; Chin-Long Wey, "A novel reconfigurable architecture of low-power unsigned multiplier for digital signal processing" Circuits and Systems, ISCAS 2005, Vol. 4, Page(s):3327 - 3330, May 2005.

[25] Calhoun, B.H.; Chandrakasan, A., "Ultra-dynamic voltage scaling using subthreshold operation and local voltage dithering in 90nm CMOS " Solid-State 
Circuits Conference, 2005. Digest of Technical Papers. ISSCC. 2005 IEEE International, Vol. 1, Page(s):300 - 599, Feb. 2005.

[26] Kang, J.-Y.; Gaudiot, J.-L.; "A Simple High-Speed Multiplier Design Computers" Computers IEEE Transactions, Vol. 55,Issue: 10, Page(s):1253 $1258,2006$.

[27] Geun Young Jeong; Ju Sung Park; Hee Chul Kang; "A Study on Multiplier Architecture Optimized for 32-bit Processor with 3-Stage Pipeline" International SoC Design Conference, October, 2004.

[28] Perri, S.; Corsonello, P.; Iachino, M.A.; Lanuzza, M.; Cocorullo, G.; "Variable precision arithmetic circuits for FPGA-based multimedia processors" Very Large Scale Integration (VLSI) Systems, Vol. 12, Issue: 9, Page(s):995 999, 2004.

[29] Haynes, S.D.; Ferrari, A.B.; Cheung, P.Y.K.; "Flexible reconfigurable multiplier blocks suitable for enhancing the architecture of FPGAs" Custom Integrated Circuits, 1999, Page(s):191 - 194, 1999.

[30] Kim, D.; Han, G. A 200 s Processing Time Smart Image Sensor for an Eye Tracker Using Pixel-Level Analog Image Processing. IEEE J. Solid-State Circuits 2009, 44, 2581C2590.

[31] Bermak, A.; Kitchen, A. A Novel Adaptative Logarithmic Digital Pixel Sensor. IEEE Photonics Technol. Lett. 2006, 18, $2147 \mathrm{C} 2149$.

[32] Chen, S.; Bermak, A.; Boussaid, F. A Compact Reconfigurable CounterMemory for Spiking Pixels. IEEE Electron Device Lett. 2006, 27, 255 C257.

[33] Bermak, A.; Yung, Y.F. A DPS Array with Programmable Resolution and Reconfigurable Conversion Time. IEEE Trans. Very Large Scale Integr. (VLSI) Syst. 2006, 18, 15C22.

[34] Chen, S.; Bermak, A. Arbitrated Time to First Spike CMOS Image Sensor with On-Chip Histogram Equalization. IEEE Trans. Very Large Scale Integr. (VLSI) Syst. 2007, 18, 346C357. 
[35] Zhang, M.; Bermak, A. Compressive Acquisition CMOS IMage Sensor: From the Algorithm to Hardware Implementation. IEEE Trans. Very Large Scale Integr. (VLSI) Syst. 2007, 18, 490C500.

[36] Campos, F.S.; Marinov, O.; Faramarzpour, N.; Saffih, F.; Jamal Deen, M.; Swart, J.W. A multisampling time-domain CMOS imager with synchronous readout circuit. Analog Integr. Circuits Signal Process. 2008, 44, 151C159.

[37] A. Kitchen, A. Bermak and A. Bouzerdoum, PWM digital pixel sensor based on asynchronous self-resetting scheme, IEEE Electron Device Letters, Volume: 25 , Issue: 7 , pp. 471-473, July 2004.

[38] Somasekhar, D.; Ye, Y.D.; Aseron, P.; Lu, S.L.; Khellah, M.M.; Howard, J.; Ruhl, G.; Karnik, T.; Borkar, S.; De, V.K.; Keshavarzi, A. 2 GHz 2Mb $2 \mathrm{~T}$ Gain CellMemoryMacroWith 128 GBytes/sec Bandwidth in a $65 \mathrm{~nm}$ Logic Process Technology. IEEE J. Solid State Circuits 2009, 44, 174C185.

[39] Amrutur, B.S.; Horowitz,M.A. A Replica Technique forWordline and Sense Control in Low-Power SRAMs. IEEE J. Solid State Circuits 1998, 33, $1208 \mathrm{C} 1219$.

[40] Rafael C.; Gonzalez; Richard Eugene Woods; "Digital Image Processing", Prentice Hall, 2008.

[41] Choy, S.K.; Tong, C.S.; " A correlated bit-plane model for wavelet subband histograms and its application to Chinese materia medica starch grains classification" Signal-Image Technologies and Internet-Based System, SITIS 2007, Page(s): 542-548, 2007.

[42] Dang, T.T.; Nguyen, S.K.; Vu, T.D.; Higuchi, S.; " A lossless coding scheme for images by using cross point regions on multiple bit planes" Imaging Systems and Techniques, IST '09. IEEE International Workshop, Page(s): 282286, May 2009.

[43] Tang Fei; Liu SunDong; Guo Sen; "A novel method of crowd estimation in public locations" BioMedical Information Engineering, FBIE 2009, Page(s): 339 - 342, Dec 2009. 
[44] Sung-Jea Ko; Sung-Hee Lee; Kyung-Hoon Lee; "Digital image stabilizing algorithms based on bit-plane matching" Consumer Electronics, IEEE Transactions, Volume: 44, Issue: 3, Page(s): 617 - 622, 1998.

[45] Chindaro, S.; Sirlantzis, K.; Fairhurst, M.C.; Hoque, S.; "Diversityperformance relationship in a handwriting recognition system based on bitplane decomposition" Frontiers in Handwriting Recognition, IWFHR-9 2004, Ninth International Workshop, Page(s): 413 - 418, 2004.

[46] Muhammad Bilal; Shahid Masud; "Efficient color space conversion using custom instruction in a RISC processor" Circuits and Systems, ISCAS 2007, IEEE International Symposium, Page(s): 1109 - 1112, May 2007.

[47] Bonney, B.; Ives, R.; Etter, D.; Yingzi Du; "Iris pattern extraction using bit planes and standard deviations" Signals, Systems and Computers, 2004, Volume: 1, Page(s): 582 - 586, Nov 2004.

[48] Sapiro, G.; Malah, D.; "Morphological image coding via bit-plane decomposition and a new skeleton representation" Electrical and Electronics Engineers in Israel, 17th Convention, Page(s): 174 - 177, August 1991.

[49] Ardizzone, E.; Dindo, H.; Mazzola, G.; "Restoration of digitized damaged photos using bit-plane slicing" Multimedia and Expo, 2007 IEEE International Conference, Page(s): 1643 - 1646, August 2007.

[50] Bishnu, A.; Bhattacharya, B.B.; "Stacked Euler Vector (SERVE) a graytone image feature based on bit-plane augmentation" Pattern Analysis and Machine Intelligence, IEEE Transactions, Volume: 29, Issue: 2, Page(s): 350 $-355,2007$.

[51] HOUGH, P. V. C. Method and means for recognizing complex patterns. U. S. Patent 3, 069 654, December 18, 1962.

[52] Illinggworth,J.and J.Kittler, "A survey of the Hough transform", Computer Vision, Graphics, and Image Processing, Vol.44, No.1, pp 87-116, 1988. 
[53] Daggu Venkateshwar Rao, Shruti Patil, Naveen Anne Babu and V Muthukumar, "Implementation and Evaluation of Image Processing Algorithms on Reconfigurable Architecture using C-based Hardware Descriptive Languages", International Journal of Theoretical and Applied Computer Sciences Vol. 1 Num. 1, pp. 9-34, 2006.

[54] Sathyanarayana, S.S.; Satzoda, R.K.; Srikanthan, T.; "Exploiting Inherent Parallelisms for Accelerating Linear Hough Transform", Image Processing, IEEE Transactions on Volume: 18, Issue: 10, Page(s): 2255 - 2264, 2009.

[55] Geninatti, S.R.; Benitez, J.I.B.; Calvio, M.H.; Mata, N.G.; Luna, J.G.; "FPGA Implementation of the Generalized Hough Transform", Reconfigurable Computing and FPGAs, Page(s): 172 - 177, 2009.

[56] Hardzeyeu, V.; Klefenz, F.; "On using the hough transform for driving assistance applications", Intelligent Computer Communication and Processing, Page(s): 91 - 98, 2008. 


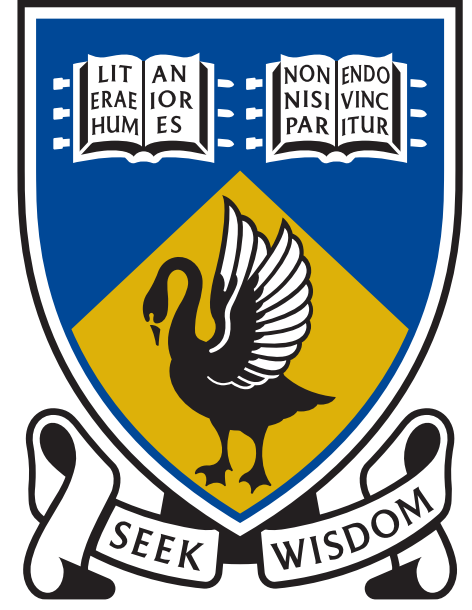

THE UNIVERSITY OF

WESTERN AUSTRALIA Achieve International Excellence 